CERN-TH/2003-307

\title{
The Magnetized Universe
}

\author{
Massimo Giovannini ${ }^{1}$ \\ CERN, Theoretical Physics Division, CH-1211 Geneva 23, Switzerland
}

\begin{abstract}
Cosmology, high-energy physics and astrophysics are converging on the study of large-scale magnetic fields. While the experimental evidence for the existence of large-scale magnetization in galaxies, clusters and superclusters is rather compelling, the origin of the phenomenon remains puzzling especially in light of the most recent observations. The purpose of the present review is to describe the physical motivations and some of the open theoretical problems related to the existence of large-scale magnetic fields.
\end{abstract}

\footnotetext{
${ }^{1}$ e-mail address: massimo.giovannini@cern.ch
} 


\section{Contents}

1 A triple point 3

2 From Alfvén to ROSAT 5

\begin{tabular}{lll}
\hline & Large-scale magnetic fields observations & $\mathbf{8}$
\end{tabular}

3.1 Zeeman splitting . . . . . . . . . . . . . . . . . . 99 9

3.2 Svnchrotron emission ................ . . . . 10

3.3 Faradav Rotation Measurements . . . . . . . . . . . . . . 12

3.4 Magnetic fields in the Milkv Wav . . . . . . . . . . . . . . . 15

3.5 Magnetic fields in external galaxies . . . . . . . . . . . . . . 20

3.6 Magnetic fields in Abell clusters . . . . . . . . . . . . . . . . . 20

3.7 Magnetic fields in superclusters . . . . . . . . . . . . . . 23

4 Globally Neutral Plasmas 24

4.1 Qualitative aspects of plasma dvnamics . . . . . . . . . . . . . 24

4.2 Kinetic and fluid descriptions . . . . . . . . . . . . . . . 26

4.3 From one-fluid equations to MHD . . . . . . . . . . . . . . 27

4.4 The Alfvén theorems . . . . . . . . . . . . . . . . . . 30

4.5 Magnetic diffusivity equation . . . . . . . . . . . . . . . 32

4.6 The dvnamo mechanism . . . . . . . . . . . . . . . . 36

5 Magnetic Fields in Curved Backgrounds 41

5.1 The standard cosmological model . . . . . . . . . . . . . . . . 41

5.1.1 Inflationarv dvnamics and its extensions . . . . . . . . 44

5.2 Gauge fields in FRW Universes . . . . . . . . . . . . . . . 48

5.3 Phvsical scales in the evolution of magnetic fields . . . . . . . 52

5.3.1 Before matter-radiation equality . . . . . . . . . . . 52

5.3 .2 After matter-radiation equality . . . . . . . . . . . 56

5.4 Charge and current densitv fluctuations . . . . . . . . . . . . . 58

5.5 Inverse cascades . . . . . . . . . . . . . . . . . . . . 62

5.6 Hvpermagnetic fields . . . . . . . . . . . . . . . . . . . . 68

5.6.1 Anomalous MHD equations . . . . . . . . . . . 69

5.6 .2 Hvpermagnetic knots . . . . . . . . . . . . . . . 73

$\begin{array}{lll}6 & \text { Two approaches to the Origin } & 77\end{array}$

6.1 Inhomogeneous MHD equations . . . . . . . . . . . . . 78

6.2 Inflationary mechanisms . . . . . . . . . . . . . . 80 
6.2 .1 Conventional inflationarv models . . . . . . . . . . 87

6.2 .2 Abelian Higgs model . . . . . . . . . . . . . . . . . . . 90

6.2 .3 Internal dimensions . . . . . . . . . . . . . . . . . . . . 91

6.2.4 String cosmological models . . . . . . . . . . . . . . . 93

6.3 Inside the Hubble radius . . . . . . . . . . . . . . . . . . . 94

6.3 .1 Phase transitions . . . . . . . . . . . . . . . . 94

6.4 Mixed mechanisms . . . . . . . . . . . . . . . . . . 97

$\begin{array}{lll}7 & \text { Effects of primordial magnetic fields } & 98\end{array}$

7.1 Electroweak epoch . . . . . . . . . . . . . . . . . . . . . . . . 99

$7.1 .1 \quad$ EW phase diagram . . . . . . . . . . . . . . . . . . . 99

7.1 .2 Barvon asvmmetrv . . . . . . . . . . . . . . . 100

7.2 Big-bang nucleosynthesis epoch . . . . . . . . . . . 102

8 CMB physics an magnetic fields 106

8.1 Large-scale magnetic fields and CMB anisotropies . . . . . . 107

8.2 Faradav rotation of CMB $\ldots \ldots \ldots \ldots \ldots$

8.3 Relic gravitational waves . . . . . . . . . . . . . . 116

9 Gravitating magnetic fields 119

10 Concluding Remarks 122

\begin{tabular}{|ll}
\hline A Complements on MHD description & 125
\end{tabular} 


\section{A triple point}

Why do we observe magnetic fields in the sky? Why do we live in a magnetized Universe? A variety of observations imply that stars, planets, galaxies, clusters of galaxies are all magnetized. The typical magnetic field strengths ${ }^{2}$ range from few $\mu \mathrm{G}$ (in the case of galaxies and galaxy clusters) to few $\mathrm{G}$ (in the case of planets, like the earth) up to $10^{12} \mathrm{G}$ (in the case of neutron stars). Physical phenomena are characterized depending upon the typical time and length scales where they take place. Magnetic fields of stars and planets are related to length-scales which are much smaller than the diameter of the Milky Way (of the order of $30 \mathrm{Kpc}$ ) or of the local supercluster. In this sense, a magnetic field of the order of $\mu \mathrm{G}$ is minute on the terrestrial scale but it is sizable over the scale of the supercluster.

In the present review only large-scale magnetic fields will be considered, i.e. magnetic fields whose typical scale exceeds the $\mathrm{AU}$ ( $1 \mathrm{AU}=$ $1.49 \times 10^{13} \mathrm{~cm}$ ). Large-scale magnetic fields must be understood and treated as an essential part of the largest structures observed in the sky. Legitimate questions arising in the study of magnetized structures concern their formation and evolution. The answers to these questions are still not completely settled. Specific observations will eventually allow to discriminate between the different competing explanations.

Magnetic fields of distant spiral galaxies are in the $\mu \mathrm{G}$ range. There is also compelling evidence of large-scale magnetic fields which are not associated with individual galaxies. This empirical coincidence reminds a bit of one of the motivations of the standard hot big-bang model, namely the observation that the light elements are equally abundant in rather different parts of our Universe. The approximate equality of the abundances implies that, unlike the heavier elements, the light elements have primordial origin. The four light isotopes $\mathrm{D},{ }^{3} \mathrm{He},{ }^{4} \mathrm{He}$ and ${ }^{7} \mathrm{Li}$ are mainly produced at a specific stage of the hot big bang model named nucleosynthesis occurring below the a typical temperature of $0.8 \mathrm{MeV}$ when neutrinos decouple from the plasma and the neutron abundance evolves via free neutron decay. The abundances calculated in the simplest big-bang nucleosythesis model agree fairly well with the astronomical observations. In similar terms it is plausible to argue

\footnotetext{
${ }^{2}$ In this review magnetic fields will be expressed in gauss. In the SI units $1 \mathrm{~T}=10^{4} \mathrm{G}$. For practical reasons, in cosmic ray physics and in cosmology it is also useful to express the magnetic field in $\mathrm{GeV}^{2}$ (in units $\hbar=c=1$ ). Recalling that the Bohr magneton is about $5.7 \times 10^{-11} \mathrm{MeV} / \mathrm{T}$ the conversion factor will then be $1 \mathrm{G}=1.95 \times 10^{-20} \mathrm{GeV}^{2}$.
} 
that large-scale magnetic fields have similar strengths at large scales because the initial conditions for their evolutions were the same, for instance at the time of the gravitational collapse of the protogalaxy. The way the initial conditions for the evolution of large-scale magnetic fields are set is generically named magnetogenesis.

There is another comparison which might be useful. Back in the seventies the so-called Harrison-Zeldovich spectrum was postulated. Later, with the developments of inflationary cosmology the origin of a flat spectrum of curvature and density profiles has been justified on the basis of a period of quasi-de Sitter expansion named inflation. It is plausible that in some inflationary models not only the fluctuations of the geometry are amplified but also the fluctuations of the gauge fields. This happens if, for instance, gauge couplings are effectively dynamical. As the Harrison-Zeldovich spectrum can be used as initial condition for the subsequent Newtonian evolution, the primordial spectrum of the gauge fields can be used as initial condition for the subsequent magnetohydrodynamical (MHD) evolution which may lead, eventually, to the observed large-scale magnetic fields.

Cosmologists and theoretical physicists would like to understand largescale magnetization in terms of symmetries which are broken. There are other two different motivations leading, independently, to the study of largescale magnetic fields. For instance it was observed long ago by Fermi that if cosmic rays are in equilibrium with the galaxy, their pressure density is comparable with the one of the magnetic field of the galaxy. Large-scale magnetic fields are also extremely relevant for astrophysics. Magnetic fields in galaxies are sufficiently intense to affect the dynamics of interstellar gas both on the galactic scale and also on smaller scales characteristic of star formation processes. This is the third motivation leading to the study of large scale-magnetic fields: the astrophysical motivation which tries to combine our knowledge of the Universe in powerful dynamical principles based on the microscopic laws of nature.

The cross-disciplinary character of the physical phenomena addressed in this review is apparent from the table of content. In Section 2 a brief historical account of this fifty years old problem will be given. Then, to clarify the nature of the various theoretical constructions, the empirical evidence of large scale magnetic fields will be discussed in Section 3. Section 4 contains some background material on the evolution of magnetic fields in globally neutral plasmas in flat space. Section 5 and 6 address the problem of the evolution of large scale magnetic fields in curved backgrounds and of their 
origin. In Section 7 the possible effects of magnetic fields on the thermodynamical history of the Universe are scrutinized. Section 8 will be concerned with the effects of large scale magnetic fields on the Cosmic Microwave Background radiation (CMB) and on the relic background of gravitational waves. In Section 9 the rôle of gravitating magnetic fields in cosmological solutions will be swiftly pointed out.

The perspective of present review is theoretical. In this sense various (very important) experimental results (for instance concerning optical polarization) will receive only a swift attention.

\section{From Alfvén to ROSAT}

In January 1949 large-scale magnetic fields had no empirical evidence. The theoretical situation can be summarized as follows:

- the seminal contributions of Alfvén [1] convinced the community that magnetic fields can have a very large life-time in a highly conducting plasma;

- using the new discoveries of Alfvén, Fermi [2] postulated the existence of a large-scale magnetic field permeating the galaxy with approximate intensity of $\mu \mathrm{G}$ and, hence, in equilibrium with the cosmic rays;

- rather surprisingly Alfvén [3] did not appreciate the implications of the Fermi idea and was led to conclude (incorrectly) that cosmic rays are in equilibrium with stars disregarding completely the possibility of a galactic magnetic field;

- in 1949 Hiltner [4] and, independently, Hall [5] observed polarization of starlight which was later on interpreted by Davis and Greenstein [6] as an effect of galactic magnetic field aligning the dust grains ${ }^{3}$.

According to the presented chain of events it is legitimate to conclude that

\footnotetext{
${ }^{3}$ It should be noticed that the observations of Hiltner [4 and Hall [5] took place from November 1948 to January 1949. The paper of Fermi [2] was submitted in January 1949 but it contains no reference to the work of Hiltner and Hall. This indicates the Fermi was probably not aware of these optical measurements.
} 
- the discoveries of Alfvén were essential in the Fermi proposal (who was already thinking of the origin of cosmic rays in 1938 before leaving Italy);

- the idea that cosmic rays are in equilibrium with the galactic magnetic fields (and hence that the galaxy possess a magnetic field) was essential in the correct interpretation of the first, fragile, optical evidence of galactic magnetization.

The origin of the galactic magnetization, according to [2, had to be somehow primordial. This idea was further stressed in two subsequent investigations of Fermi and Chandrasekar [7, 8, who tried, rather ambitiously, to connect the magnetic field of the galaxy to its angular momentum.

In the fifties various observations on polarization of Crab nebula suggested that the Milky Way is not the only magnetized structure in the sky. The effective new twist in the observations of large-scale magnetic fields was the development (through the fifties and sixties) of radio-astronomical techniques. From these measurements, the first unambiguous evidence of radio-polarization from the Milky Way (MW) was obtained [10].

It was also soon realized that the radio-Zeeman effect (counterpart of the optical Zeeman splitting employed to determine the magnetic field of the sun) could offer accurate determination of (locally very strong) magnetic fields in the galaxy. The observation of Lyne and Smith [11] that pulsars could be used to determine the column density of electrons along the line of sight opened the possibility of using not only synchrotron emission as a diagnostic of the presence of a large-scale magnetic field, but also Faraday rotation. In the seventies all the basic experimental tools for the analysis of galactic and extra-galactic magnetic fields were present. Around this epoch also extensive reviews on the experimental endeavors started appearing and a very nice account could be found, for instance, in the review of Heiles [12].

It became gradually clear in the early eighties, that measurements of large-scale magnetic fields in the MW and in the external galaxies are two complementary aspects of the same problem. While MW studies can provide valuable informations concerning the local structure of the galactic magnetic field, the observation of external galaxies provides the only viable tool for the reconstruction of the global features of the galactic magnetic fields. As it will be clarified in the following Sections, the complementary nature of global and local morphological features of large-scale magnetization may become, sometimes, a source of confusion. 
Since the early seventies, some relevant attention has been paid not only to the magnetic fields of the galaxies but also to the magnetic fields of the clusters. A cluster is a gravitationally bound system of galaxies. The local group (i.e. our cluster containing the MW, Andromeda together with other fifty galaxies) is an irregular cluster in the sense that it contains fewer galaxies than typical clusters in the Universe. Other clusters (like Coma, Virgo) are more typical and are then called regular or Abell clusters. As an order of magnitude estimate, Abell clusters can contain $10^{3}$ galaxies.

In the nineties magnetic fields have been measured in single Abell clusters but around the turn of the century these estimates became more reliable thanks to improved experimental techniques. In order to estimate magnetic fields in clusters, an independent knowledge of the electron density along the line of sight is needed (see Sec. 3). Recently Faraday rotation measurements obtained by radio telescopes (like VLA ${ }^{4}$ ) have been combined with independent measurements of the electron density in the intra-cluster medium. This was made possible by the maps of the x-ray sky obtained with satellites measurements (in particular ROSAT ${ }^{5}$ ). This improvement in the experimental capabilities seems to have partially settled the issue confirming the measurements of the early nineties and implying that also clusters are endowed with a magnetic field of $\mu \mathrm{G}$ strength which is not associated with individual galaxies.

The years to come are full of interesting experimental questions to be answered. These questions, as it will be discussed in the following, may have a rather important theoretical impact both on the theory of processes taking place in the local universe (like ultra-high energy cosmic ray propagation) and on the models trying to explain the origin of large scale magnetic fields. Last but not least, these measurements may have an impact on the physics of CMB anisotropies and, in particular, on the CMB polarization. In fact, the same mechanism leading to the Faraday rotation in the radio leads to a Faraday rotation of the $\mathrm{CMB}$ provided the $\mathrm{CMB}$ is linearly polarized. One of the important questions to be answered is, for instance, the nature and strength of the supercluster magnetic field and now more careful statistical studies are starting also along this important direction. Superclusters are gravitationally bound systems of clusters. An example is the local supercluster formed

\footnotetext{
${ }^{4}$ The Very Large Array Telescope, consists of 27 parabolic antennas spread over a surface of $20 \mathrm{~km}^{2}$ in Socorro (New Mexico)

${ }^{5}$ The ROegten SATellite (flying from June 1991 to February 1999) provided maps of the $\mathrm{x}$-ray sky in the range $0.1-2.5 \mathrm{keV}$. A catalog of x-ray bright Abell clusters was compiled.
} 
by the local group and by the VIRGO cluster. Together ROSAT, various observations with the EINSTEIN, EXOSAT, and GINGA satellites showed the presence of hot diffuse gas in the Local Supercluster. The estimated magnetic field in this system is, again, of the order of the $\mu \mathrm{G}$ but the observational evidence is still not conclusive. Another puzzling evidence is the fact that Lyman- $\alpha$ systems (with red-shifts $z \sim 2.5$ ) are endowed with a magnetic field [13].

From the historical development of the subject a series of questions arises naturally:

- what is the origin of large-scale magnetic fields?

- are magnetic fields primordial as assumed by Fermi more than 50 years ago?

- even assuming that large-scale magnetic fields are primordial, is there a theory for their generation?

\section{Large-scale magnetic fields observations}

Before describing the theory of something (not of everything) it is highly desirable, in natural sciences, to understand in some detail the empirical evidence of the subject under discussion. While technical accounts of the various experimental techniques exist already 12, 13, 14, 15, 16, 17, 18 it seems useful, in the present context, to give an account of the experimental situation by emphasizing the physical principles of the various measurements. There are valuable books dealing directly with large-scale magnetism [19, 20, 21]. Furthermore excellent reviews of the morphological features of large scale magnetism can be found in [14, 15, 16, 17]. Even if the present review will not be concerned with planetary magnetic fields, analogies with physical situations arising closer to the earth are often useful and, along this perspective, magnetic field observations in the solar system have been recently reviewed 22 .

Magnetic fields observed in the Universe have a homogeneous (or uniform) component and a non-uniform component. It is then useful, for the purposes of this Section, to write, in a schematic notation, that

$$
B_{\text {tot }}=\bar{B}+\delta B
$$


where $\bar{B}$ is the uniform component and $\delta B$ the non-uniform component. Both components are phenomenologically very relevant. Different experimental techniques can probe different components of large-scale magnetic fields (i.e. either the total magnetic field or its homogeneous component). Another important distinction is between $B_{\perp}$ and $B_{\|}$: there are measurements (like synchrotron emission) which are sensitive to $B_{\perp}$ i.e. the transverse component of the magnetic field; there are other measurements (like the Faraday Rotation measure or the Zeeman splitting of spectral lines) which are sensitive to $B_{\|}$i.e. the magnetic field along the line of sight.

\subsection{Zeeman splitting}

In order to measure large scale magnetic fields, one of the first effects coming to mind, is the Zeeman effect. The energy levels of an hydrogen atom in the background of a magnetic field are not degenerate. The presence of a magnetic field produces a well known splitting of the spectral lines:

$$
\Delta \nu_{Z}=\frac{e \bar{B}_{\|}}{2 \pi m_{e}} .
$$

where $\bar{B}_{\|}$denotes the uniform component of the magnetic field along the line of sight. From the estimate of the splitting, the magnetic field intensity can be deduced. Indeed this technique is the one commonly employed in order to measure the magnetic field of the sun [22]. The most common element in the interstellar medium is neutral hydrogen, emitting the celebrated $21-\mathrm{cm}$ line (corresponding to a frequency of $1420 \mathrm{MHz}$ ). If a magnetic field of $\mu \mathrm{G}$ strength is present in the interstellar medium, according to Eq. (3.2), an induced splitting, $\Delta \nu_{Z} \sim 3 \mathrm{~Hz}$, can be estimated. Zeeman splitting of the $21-\mathrm{cm}$ line generates two oppositely circular polarized spectral lines whose apparent splitting is however sub-leading if compared to the Doppler broadening. In fact, the atoms and molecules in the interstellar medium are subjected to thermal motion and the amount of induced Doppler broadening is roughly given by

$$
\Delta \nu_{\text {Dop }} \sim\left(\frac{v_{\text {th }}}{c}\right) \nu
$$

where $v_{\text {th }}$ is the thermal velocity $\propto \sqrt{T / m}$ where $m$ is the mass of the atom or molecule. The amount of Doppler broadening is $\Delta \nu_{\text {Dop }} \sim 30 \mathrm{kHz}$ which is much larger than the Zeeman splitting which should be eventually detected. 
Zeeman splitting of molecules and recombination lines should however be detectable if the magnetic field strength gets larger with the density. Indeed in the interstellar medium there are molecules with an unpaired electron spin. From Eq. (3.2) it is clear that a detectable Zeeman splitting (i.e. comparable or possibly larger than the Doppler broadening) can be generically obtained for magnetic fields where $\bar{B}_{\|} \simeq 10^{-3} \mathrm{G}$, i.e. magnetic fields of the order of the $\mathrm{m}$ G. Molecules with an unpaired electron spin include $\mathrm{OH}, \mathrm{CN}, \mathrm{CH}$ and some other. In the past, for instance, magnetic fields have been estimated in $\mathrm{OH}$ clouds (see 12 and references therein). Magnetic fields of the order of $10 \mathrm{~m} \mathrm{G}$ have been detected in interstellar $\mathrm{H}_{2} \mathrm{O}$ maser clumps (with typical densities $\mathcal{O}\left(10^{10} \mathrm{~cm}^{-3}\right)$ ) 23]. More recently attempts of measuring magnetic fields in $\mathrm{CN}$ have been reported 24. The possible caveat with this type of estimates is that the measurements can only be very local: the above mentioned molecules are much less common than neutral hydrogen and are localized in specific regions of the interstellar medium. In spite of this caveat, Zeeman splitting measurements can provide reliable informations on the local direction of the magnetic field. This determination is important in order to understand the possible origin of the magnetic field. This aspect will be discussed, in more detail, when describing the magnetic field of the Milky Way.

\subsection{Synchrotron emission}

The first experimental evidence of the existence of large-scale magnetic fields in external galaxies came, historically, from the synchrotron emission of Crab nebula [25]. The emissivity formula (i.e. the energy emitted from a unit volume, per unit time, per unit frequency interval and per unit solid angle) for the synchrotron is a function of $B_{\perp}$ and and of the relativistic electron density, namely

$$
\mathcal{W}\left(B_{\perp}, \nu\right)=\mathcal{W}_{0} n_{0} B_{\mathrm{tot}, \perp}^{(1+\alpha) / 2} \nu^{(1-\alpha) / 2}
$$

where $\nu$ is the frequency, $\mathcal{W}_{0}$ is a proportionality constant depending only upon the spectral index $\alpha$ which also determines the (isotropic) relativistic electron number density distribution for electrons with energies in the range $d E$ :

$$
n_{\mathrm{e}} d E=n_{0} E^{-\alpha} d E
$$


A useful estimate for the maximal emission from electrons of energy $E$ is given by [21]

$$
\left(\frac{\nu}{\mathrm{MHz}}\right) \simeq 15\left(\frac{B_{\mathrm{tot}, \perp}}{\mu \mathrm{G}}\right)\left(\frac{E}{\mathrm{GeV}}\right)^{2} .
$$

In generic terms the synchrotron emission is then sensitive to the total transverse magnetic field. In oversimplified terms the measurement proceeds in three steps:

- estimate of the emission intensity;

- from the estimate of the emission intensity, defining as $L$ the typical size of the source, the quantity $B_{\mathrm{tot}, \perp}^{(\alpha+1) / 2} \mathcal{W}_{0} L$ can be deduced;

- the magnetic field can then be obtained by specifying the electron density distribution of the source.

While the second step is rather well defined ( $\alpha$ can indeed be determined from the observed emission spectrum ${ }^{6}$ ) the third step has to be achieved in a model-dependent way. The relativistic electron density is sometimes estimated using equipartition, i.e. the idea that magnetic and kinetic energy densities may be, after all, comparable. Equipartition is not always an empirical evidence, but can certainly be used as a working hypothesis which may or may not be realized in the system under consideration. For instance equipartition probably holds for the Milky Way but it does not seem to be valid in the Magellanic Clouds [26. The average equipartition field strengths in galaxies ranges from the $4 \mu \mathrm{G}$ of M33 up to the $19 \mu \mathrm{G}$ of NGC2276 [27, 28.

In order to illustrate the synchrotron emission take for instance the MW. Away from the region of the galactic plane synchrotron emission is the dominant signal of the galaxy for frequencies below $5 \mathrm{GHz}$. Between 1.4 and $5 \mathrm{GHz}$ the spectral dependence is $\mathcal{O}\left(\nu^{-0.9}\right)$. It should be noticed that the spectral index of synchrotron emission may change in different frequency ranges and also in different regions of the sky due to the variation of the magnetic field [29. This aspect is crucial in the experimental analysis of CMB anisotropy experiments in the $\mathrm{GHz}$ region. In fact, in the context of $\mathrm{CMB}$ studies the synchrotron is a foreground which should be appropriately subtracted. The way this is done is by extrapolation of lower frequency measurements. The

\footnotetext{
${ }^{6}$ Typical galactic values of $\alpha$ are between 2.4 and 2.8. An important caveat to this statement, relevant for foreground extraction (for instance in studies of CMB anisotropies) is that the synchrotron spectrum may have a break.
} 
problem of proper subtraction of synchrotron foreground is particularly acute in the case of the PLANCK mission ${ }^{7}$

The synchrotron has an intrinsic polarization which can give the orientation of the magnetic field, but not the specific sign of the orientation vector. The strength of synchrotron polarization is proportional to $\left|\bar{B}_{\perp} / B_{\text {tot, } \perp}\right|^{2}$, i.e. the ratio between the magnetic energy densities of the uniform and total magnetic field. Thus, according to Eq. (3.1), the synchrotron polarization is sensitive to the random component of the large-scale magnetic field.

\subsection{Faraday Rotation Measurements}

To infer the magnitude of the magnetic field strength Faraday effect has been widely used. When a polarized radio wave passes through a region of space of size $\delta \ell$ containing a plasma with a magnetic field the polarization plane of the wave gets rotated by an amount

$$
\delta \phi \propto \omega_{\mathrm{B}}\left(\frac{\omega_{\mathrm{p}}}{\omega}\right)^{2} \delta \ell
$$

which is directly proportional to the square of the plasma frequency ${ }^{8} \omega_{\mathrm{p}}$ (and hence to the electron density) and to the Larmor frequency $\omega_{\mathrm{B}}$ (and hence to the magnetic field intensity). A linear regression connecting the shift in the polarization plane and the square of the wavelength $\lambda$, can be obtained:

$$
\phi=\phi_{0}+\mathrm{RM} \lambda^{2}
$$

By measuring the relation expressed by Eq. (3.8) for two (or more) separate (but close) wavelengths, the angular coefficient of the regression can be obtained and it turns out to be

$$
\frac{\Delta \phi}{\Delta \lambda^{2}}=811.9 \int\left(\frac{n_{e}}{\mathrm{~cm}^{-3}}\right)\left(\frac{\bar{B}_{\|}}{\mu \mathrm{G}}\right) d\left(\frac{\ell}{\mathrm{kpc}}\right),
$$

in units of $\mathrm{rad} / \mathrm{m}^{2}$ when all the quantities of the integrand are measured in the above units. The explicit dependence of the red-shift can be also easily included in Eq. (3.9). Notice, in general terms that the RM is an integral

\footnotetext{
${ }^{7}$ The satellite mission PLANCK EXPLORER will provide, after 2008, full sky maps in nine frequency channels ranging from 30 to $900 \mathrm{GHz}$.

${ }^{8}$ See Eq. (4.5) in the following Section.
} 
over distances. Thus the effect of large distances will reflect in high values of the RM. Furthermore, the Faraday effect occurs typically in the radio (i.e. $\mathrm{cm}<\lambda<\mathrm{m}$ ), however, some possible applications of Faraday effect in the microwave can be also expected (see Section 8 of the present review).

The shift in the polarization plane should be determined with an accuracy greater than $\delta \phi \sim \pm \pi$. Otherwise ambiguities may arise in the determination of the angular coefficient appearing in the linear regression of Eq. (3.8). This aspect is illustrated in Fig. 11 which is rather standard but it is reproduced here in order to stress the possible problems arising in the physical determination of the RM if the determination of the shift in the polarization plane is not accurate.

The RM defined in Eq. (3.9) not only the magnetic field (which should be observationally estimated), but also the column density of electrons. From the radio-astronomical observations, different techniques can be used in order to determine $n_{e}$. One possibility is to notice that in the observed Universe there are pulsars. Pulsars are astrophysical objects emitting regular pulses of electromagnetic radiation with periods ranging from few milliseconds to few seconds. By comparing the arrival times of different radio pulses at different radio wavelengths, it is found that signals are slightly delayed as they pass through the interstellar medium exactly because electromagnetic waves travel faster in the vacuum than in an ionized medium. Hence, from pulsars the column density of electrons can be obtained in the form of the dispersion measure, i.e. $D M \propto \int n_{e} d \ell$. Dividing the RM by DM, an estimate of the magnetic field can be obtained. Due to their abundance, pulsars lead to the best determination of the magnetic field in the galactic disk [30. In Fig. 2 (adapted from [31]) a map of the antisymmetric RM sky is reported. In the picture the open circles denote negative RM while filled circles denote positive RM. The size of the circle is proportional to the magnitude of the RM. The convention is, in fact, to attribute negative RM to a magnetic field directed away from the observer and positive RM if the magnetic field is directed toward the observer.

As in the case of synchrotron emission also Faraday rotation measurements can be used as a diagnostic for foreground contamination. The idea would be, in this context to look for cross-correlations in the Faraday rotation measure of extra-galactic sources and the measured microwave signal at the same angular position. A recent analysis has been recently reported 32 .

If magnetic field or the column density change considerably over the integration path of Eq. (3.9) one should probably define and use the two-point 


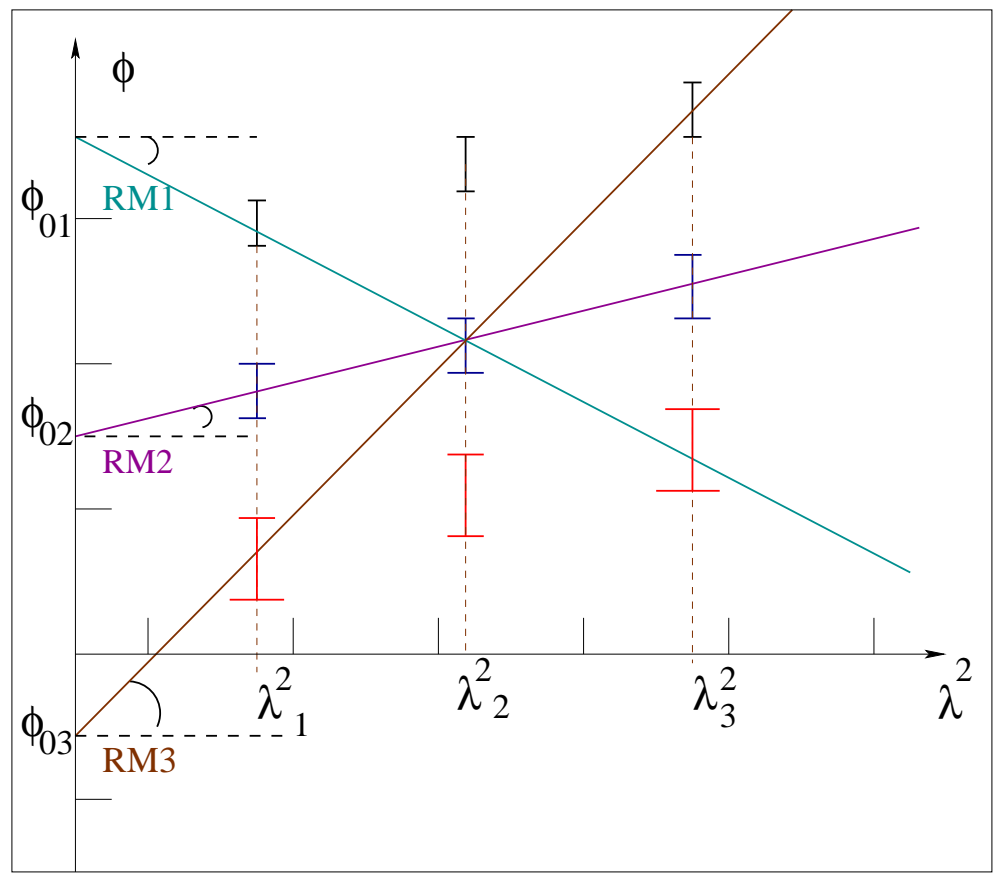

Figure 1: The possible ambiguities arising in practical determinations of the RM are illustrated. The RM is the angular coefficient of the linear regression expressed by Eq. (3.8). Clearly it is not necessary to know the initial polarization of the source to determine the slope of a straight line in the $\left(\phi, \lambda^{2}\right)$ plane, but it is enough to measure $\phi$ at two separate wavelength. However, if the accuracy in the determination of $\phi$ is of the order of $\pi$ the inferred determination of the angular coefficient of the linear regression (3.8) is ambiguous. 


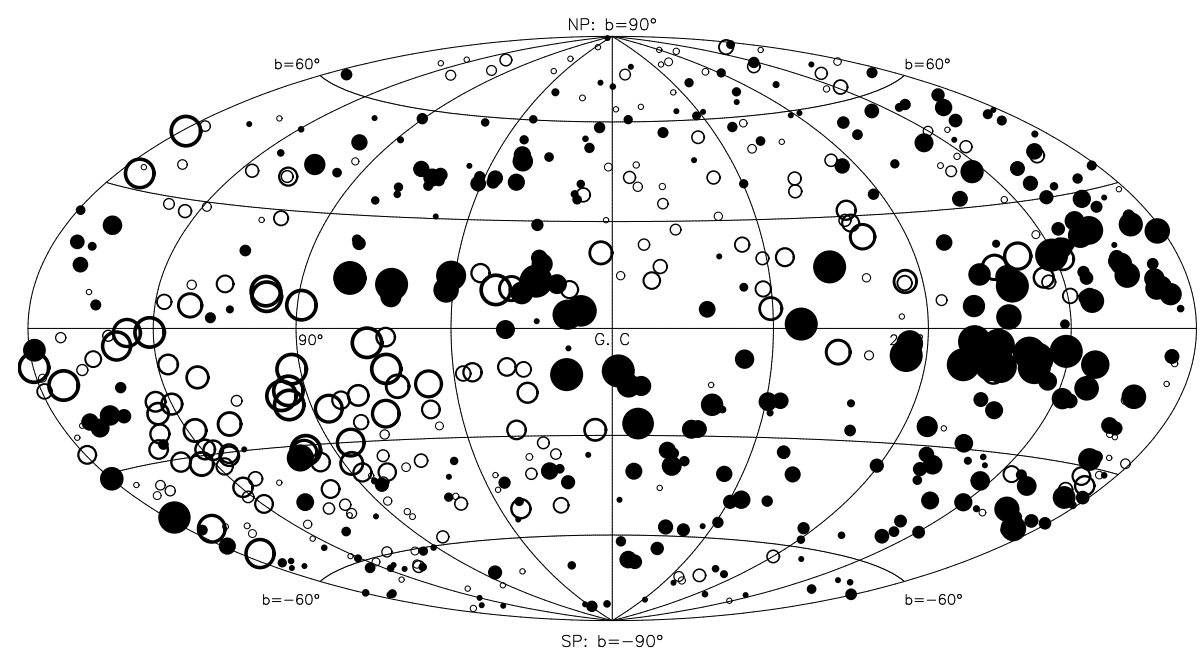

Figure 2: The filtered RM distribution of extragalactic radio sources. The antisymmetric distribution is clear especially from the inner galactic quadrant. This picture is adapted from [31].

function of the RM, i.e.

$$
\mathcal{C}(\vec{r})=\langle\operatorname{RM}(\vec{x}+\vec{r}) \operatorname{RM}(\vec{x})\rangle
$$

The suggestion to study the mean-squared fluctuation of the RM was proposed [33, 34. More recently, using this statistical approach particularly appropriate in the case of magnetic fields in clusters (where both the magnetic field intensity and the electron density change over the integration path), Newmann, Newmann and Rephaeli [35] quantified the possible (statistical) uncertainty in the determination of cluster magnetic fields (this point will also be discussed later). The rather ambitious program of measuring the $R M$ power spectrum is also pursued [36, 37]. In [38] the analysis of correlations in the RM has been discussed.

\subsection{Magnetic fields in the Milky Way}

Since the early seventies [12, 39] the magnetic field of the Milky way (MW) was shown to be parallel to the galactic plane. RM derived from pulsars allow consistent determinations of the magnetic field direction and intensity 40, 41. In the Milky Way, the uniform component of the magnetic field is in the 
plane of the galactic disk and it is thought to be directed approximately along the spiral arm. There is, though, a slight difference between the northern and southern hemisphere. While in the southern hemisphere the magnetic field is roughly $2 \mu \mathrm{G}$, the magnetic field in the northern hemisphere is three times smaller than the one of the southern hemisphere. The magnetic field of the MW is predominantly toroidal ${ }^{9}$. However, looking at the center of the galaxy, a poloidal component (typically $1 / 3$ of the toroidal one) has been detected. In fact, in the central $100 \mathrm{pc}$ of the MW there are in fact pooloidal (dipole) fields whose origin is probably primordial 42 ( see also [43. 44]). The reason for this statement is that through the usual plasma physics mechanisms (like the dynamo theory to be discussed later in Section 4) it is hard to account for a sizable poloidal component, even if localized in the central region of the MW.

The common practice is to classify large-scale magnetic fields in spiral galaxies according to the parity of the toroidal field under reflections of the azimuthal angle [21, 14]. According to some authors this distinction palys a crucial rôle in order to assess the primordial (or non-primordial ) nature of the observed field (see, for instance, [15, 18]). In short, the virtues and limitations of the previous distinction can be summarized as follows. Suppose to plot the toroidal component of the measured magnetic field (for instance of the MW) as a function of the azimuthal angle $\theta$. This is, in fact, equivalent, to plot the $R M(\theta)$ for instance near the equatorial plane of the galaxy. Then two qualitatively different situations can arise. In the first case $R M(\theta)$ upon rotation of $\pi$ around the galactic center is even and this corresponds to axisymmetric spiral galaxies (ASS). In the second case $R M(\theta)$ upon rotation of $\pi$ around the galactic center is odd and this corresponds to bisymmetric spiral galaxies (BSS). This sharp distinction is invalidated by two experimental facts. First of all there may be extra phases so that it is not clear, in practice, if $R M(\theta)$ is, predominantly, even or odd. In spite of possible ambiguities (which are, however, an experimental problem) the distinction may be very useful: if the magnetic fields would originate through some dynamo amplification the preferred configuration will be of the ASS-type and not of the BSS-type. There are strong indications that the MW is a BSS spiral 30, 31]. A related issue is the reversal of the magnetic field from one spiral arm to the other. In [31] 63 rotation measures from polarization observations

\footnotetext{
${ }^{9}$ Recall that the toroidal field is defined as the vector sum of the radial and azimuthal components, i.e. $\vec{B}_{\text {toroidal }}=B_{\theta} \hat{e}_{\theta}+B_{r} \hat{e}_{r}$.
} 
of southern pulsars have been reported. For the galactic disk, convincing evidence of field reversal near the Perseus arm is presented. In the solar circle three reversals are observed: near the Carina-Sagittarius arm, near the CruxScutum arm, and possibly a third one in the Norma arm. These reversals are claimed to be consistent with BSS models.

Since this is a relevant point, the possible controversies arising in the analysis of the magnetic field of the MW will now be swiftly mentioned. It is appropriate, for this purpose, to give a geometrical characterization of the arm structure of the MW 45. The spiral structure of the galaxy can be described in terms of a two-dimensional coordinate system $(x, y)$ whose origin $(0,0)$ is at the galactic center. In this coordinate system the sun is located at $(0,8)$ where the coordinates are expressed in kpc. For each spiral arm the equation reduces to four curves, each rotated by $\pi / 2$ of the form:

$$
x=r \cos \theta, \quad y=r \sin \theta, \quad r=r_{0} e^{\kappa\left(\theta-\theta_{0}\right)} .
$$

In Eq. (3.11) $r_{0} \sim 2.3 \mathrm{kpc}, \theta_{0} \sim 0,-\pi / 2,-\pi,-3 / 2 \pi, \kappa \sim 0.21$. In Fig. 3 the map of the spiral arms is illustrated according to the model of Eq. (3.11). Fig. 3 is adapted from the recent paper of Vallée [45]. The region with dashed lines is poorly known from the observational point of view ("zona galactica incognita" in the terminology of Vallée [45]). In terms of Fig. 3 the basic question related to the magnetic field structure concerns the relative orientation of the magnetic field direction between one arm and the nearest one. As mentioned above 30, 31] some studies suggest that three reversals are present. Some other studies [45] based on a statistical re-analysis of the most recent data from 1995 to 2001 suggest that only one reversal is observed. The two large arrows in Fig. 3 represent the field directions in the Carina-Sagittarius and in the Perseus arms. In [45] it has been argued that the magnetic field of the MW has the structure of axisymmetric spiral. However, the presence of one field reversal seems to be not fully consistent with this interpretation.

Recently [46] Han, Manchester, Lyne and Qiao, using the Parkes multibeam pulsar survey, provided further observational support for the detection of a counterclockwise magnetic field in the Norma spiral arm. The morphological properties of the magnetic field in the Carina-Sagittarius and in the Crux-Scutum arms have been confirmed [46]. These results were obtained from the analysis of pulsar rotation measures.

If the magnetic field of the MW flips its direction from one spiral arm to the other, then, as pointed out by Sofue and Fujimoto [47] the galactic 


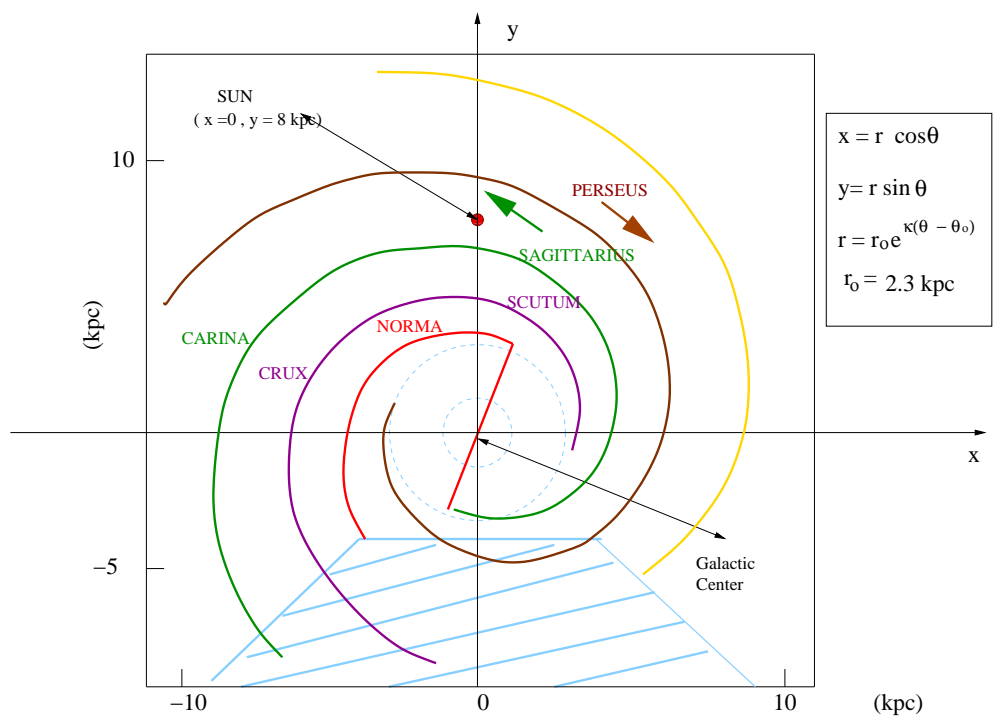

Figure 3: The map of the MW is illustrated. Following [45] the origin of the two-dimensional coordinate system are in the Galactic center. The two large arrows indicate one of the possible (3 or 5) field reversals observed so far. The field reversal indicated in this figure is the less controversial one.

magnetic field should probably be associated with a BSS model. In the SofueFujimoto model the radial and azimuthal components of the magnetic field in a bisymmetric logarithmic spiral configuration is given through

$$
\begin{aligned}
& B_{r}=f(r) \cos \left(\theta-\beta \ln \frac{r}{r_{0}}\right) \sin p, \\
& B_{\theta}=f(r) \cos \left(\theta-\beta \ln \frac{r}{r_{0}}\right) \cos p,
\end{aligned}
$$

where $r_{0} \sim 10.5 \mathrm{kpc}$ is the galactocentric distance of the maximum of the field in our spiral arm, $\beta=1 / \tan p$ and $p$ is the pitch angle of the spiral. The smooth profile $f(r)$ can be chosen in different ways. A motivated choice is [48, 49] (see also [47])

$$
f(r)=3 \frac{r_{1}}{r} \tanh ^{3}\left(\frac{r}{r_{2}}\right) \mu \mathrm{G},
$$

where $r_{1}=8.5 \mathrm{kpc}$ is the distance of the Sun to the galactic center and $r_{2}=2$ kpc. The original model of SF does not have dependence in the $z$ direction, 
however, the $z$ dependence can be included and also more complicated models can be built [4]. Typically, along the $z$ axis, magnetic fields are exponentially suppressed as $\exp \left[-z / z_{0}\right]$ with $z_{0} \sim 4 \mathrm{kpc}$.

In the BSS model there are then three issues to be determined in order to specify the parameters [50]:

- the number and location of field reversals;

- the value of the pitch angle $p$ introduced in Eq. (3.12);

- the field strength in the disk.

The answers to these three questions are, in short, the following:

- 3 to 5 field reversals have been claimed;

- the pitch angle is determined to be $p=-8.2^{0} \pm 0.5^{0}$ [31, 50];

- the strength of the regular field is, as anticipated $1.8 \pm 0.3 \mu \mathrm{G}$.

It is interesting to notice that the total field strength, in the notation of Eq. (3.1) can reach even $6 \mu \mathrm{G}$ indicating, possibly, a strong stochastic component. Differently from other spirals, the Milky Way has also a large radio halo. The radio-halo indicates the large-scale height of the magnetic field and its origin is unclear As far as the stochastic component of the galactic magnetic field is concerned, the situation seems to be, according to the reported results, still unclear 51, 52, 53. It is, at present, not fully understood if the stochastic component of the galactic magnetic field is much smaller than (or of the same order of) the related homogeneous part as implied by the estimates of the total field strength.

The structure of magnetic fields can be relevant when investigating the propagation of high-energy protons [54, 55] as noticed already long ago [56]. This aspect leads to crucial (and structural) ambiguities in the analysis of the propagation of charged particles of ultra-high energy.

The current observations of ultrahigh energy cosmic rays (UHECR) do not lead to a firm evidence of the existence of a cutoff of the cosmic ray spectrum between $10^{19}$ and $10^{20} \mathrm{eV}$. This is the celebrated Greisen, Zatsepin, Kuzmin (GZK) cutoff [57, 58]. If the source distribution of UHECR is isotropic and homogeneous because photoproduction interactions on the microwave background, then the GZK cutoff should be present. The isotropization of UHECR can be explained by scattering in large magnetic fields. This 
suggestion can be achieved in the presence of a sizable galactic halo. Magnetic fields may change not only the local intensity of UHECR but also their energy spectrum [59]. Various papers analyzed the acceleration and propagation of UHECR in magnetized structures (see, for instance, [60, 61, 62. and references therein).

\subsection{Magnetic fields in external galaxies}

An excellent presentation of the evidence of cosmic magnetism in nearby galaxies has been given by Beck et al. 15] and, in a more concise form, by Widrow 63. The past few years have witnessed a long discussion on the primordial nature of magnetic fields in nearby galaxies whose features are partially reported in [15. In analogy with the problem discussed in the case

of the MW two different opinions have been confronted. Theorists believing the primordial nature of the galactic magnetic field supported the BSS model. On the contrary, dynamo theorists supported the conclusion that most of the nearby galaxies are ASS. As previously described, the variation of the $R M$ as a function of the azimuthal angles has been used in order to distinguish ASS from BSS [64, 65]. The simplistic summary of the recent results is that there are galaxies where BSS structure dominates (like M81,M51) 66]. At the same time in some galaxies the ASS dominates [67.

\subsection{Magnetic fields in Abell clusters}

Already the short summary of the main experimental techniques used for the detection of large scales magnetic fields shows that there may be problems in the determination of magnetic fields right outside galaxies. There magnetic fields are assumed to be often of $\mathrm{nG}$ strength. However, due to the lack of sources for the determination of the column density of electrons, it is hard to turn the assumption into an experimental evidence. Magnetic fields in clusters have been recently reviewed by Giovannini 68 and, more extensively, by Carilli and Taylor [69.

Since various theoretical speculations suggest that also clusters are magnetized, it would be interesting to know if regular Abell clusters posses large scale magnetic fields. Different results in this direction have been reported [70, 71, 72] (see also [79]). Some studies during the past decade [70, 71] dealt mainly with the case of a single cluster (more specifically the Coma cluster). The idea was to target (with Faraday rotation measurements) radio sources 
inside the cluster. However, it was also soon realized that the study of many radio sources inside different clusters may lead to experimental problems due to the sensitivity limitations of radio-astronomical facilities. The strategy is currently to study a sample of clusters each with one or two bright radiosources inside.

In the past it was shown that regular clusters have cores with a detectable component of RM [79, 72]. Recent results suggest that $\mu$ Gauss magnetic fields are indeed detected inside regular clusters [73. Inside the cluster means in the intra-cluster medium. Therefore, these magnetic fields cannot be associated with individual galaxies.

Regular Abell clusters with strong x-ray emission were studied using a twofold technique [73, 74]. From the ROSAT full sky survey, the electron density has been determined [75]. Faraday RM (for the same set of 16 Abell clusters) has been estimated through observations at the VLA radio-telescope.

The amusing result (confirming previous claims based only on one cluster [70. 71]) is that x-ray bright Abell clusters possess a magnetic field of $\mu$ Gauss strength. The clusters have been selected in order to show similar morphological features. All the 16 clusters monitored with this technique are at low red-shift $(z<0.1)$ and at high galactic latitude $\left(|b|>20^{0}\right)$.

These recent developments are rather promising and establish a clear connection between radio-astronomical techniques and the improvements in the knowledge of $\mathrm{x}$-ray sky. There are various satellite missions mapping the x-ray sky at low energies (ASCA, CHANDRA, NEWTON ${ }^{10}$ ). There is the hope that a more precise knowledge of the surface brightness of regular clusters will help in the experimental determination of large scale magnetic fields between galaxies. It should be however mentioned that evidence for the presence of relativistic electrons and magnetic fields in clusters was directly available even before, from measurements of extended regions of radio synchrotron emission (for frequencies $10^{-2}<\nu<1 \mathrm{GHz}$ ) [76].

It is interesting to notice that intra-cluster magnetic fields of $\mu \mathrm{G}$ strength can induce Faraday rotation on CMB polarization. By combining informations from Sunyaev-Zeldovich effect and X-ray emission from the same clusters, it has been recently suggested that a richer information concerning electron column density can be obtained [77]. In Fig. 团 the results reported

\footnotetext{
${ }^{10}$ ASCA is operating between 0.4 AND $10 \mathrm{keV}$ and it is flying since February 1993. CHANDRA (NASA mission) and NEWTON (ESA mission) have an energy range comparable with the one of ASCA and were launched, almost simultaneously, in 1999.
} 
in [73] are summarized. In Fig. [the RM of the sample of x-ray bright Abell clusters is reported after the subtraction of the RM of the galaxy. At high galactic latitude (where all the observed clusters are) the galactic contribution is rather small and of the order of $9.5 \mathrm{rad} / \mathrm{m}^{2}$.

The results reported in [72] In Fig. [4 the open points represent sources

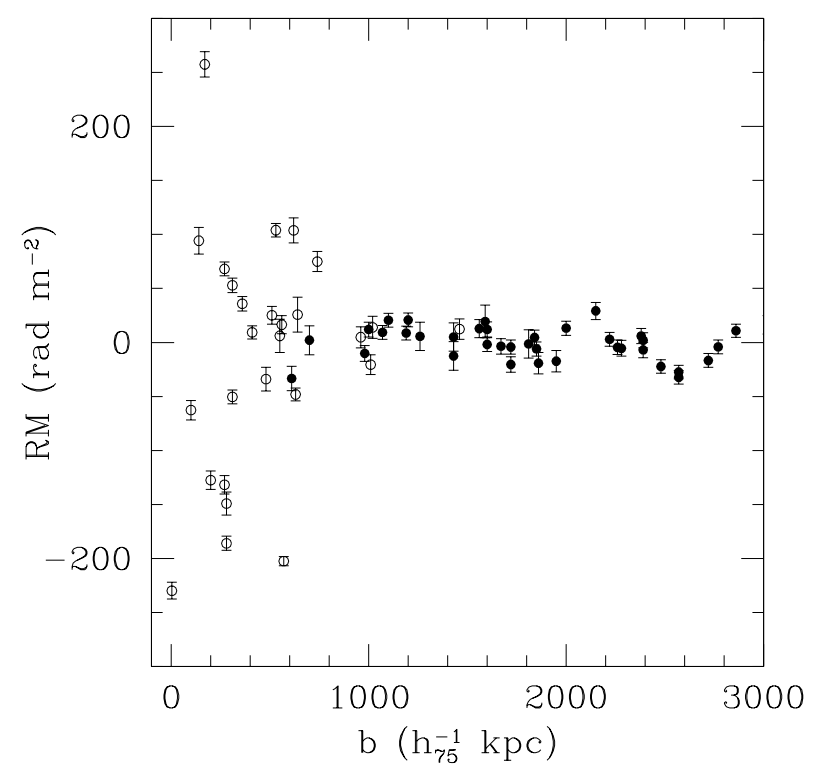

Figure 4: From Ref. 73] the RM deduced from a sample of $16 \mathrm{X}$-ray bright Abell clusters is reported as a function of the source impact parameter.

viewed through the thermal cluster gas, whereas the full points represent control sources at impact parameters larger than the cluster gas. The excess in RM attributed to clusters sources is clearly visible.

Using the described techniques large scale magnetic fields can be observed and studied in external galaxies, in clusters and also in our own galaxy. While the study of external galaxies and clusters may provide a global picture of magnetic fields, the galactic observations performed within the Milky Way are more sensitive to the local spatial variations of the magnetic field. For this reasons local and global observations are complementary. The flipped side of the coin, as we will see in the second part of the present Section, is that the global structure of the magnetic field of our galaxy is not known directly and to high precision but it is deduced from (or corroborated by) 
the global knowledge of other spiral galaxies.

\subsection{Magnetic fields in superclusters}

Magnetic fields in the local supercluster are notoriously difficult to measure. In the absence of direct measurements it is the opinion of the author that these fields cannot be assumed to be small, i.e. $\ll \mu \mathrm{G}$. In this situation it seems useful to consider the possibility that these fields are large. In [55] this point of view has been taken in connection with the propagation of the most energetic cosmic rays. This approach is desirable since the (few) reliable claims of measurements of large-scale magnetic fields in the Local Supercluster seem to indicate that these fields are rather strong and more in the range of the $\mu \mathrm{G}$ than in the range of the $\mathrm{n} G$.

Recently [8] the possible existence, strength and structure of magnetic fields in the intergalactic plane, within the Local Supercluster, has been scrutinized. The local supercluster is centered approximately at the VIRGO cluster, about $18 \mathrm{Mpc}$ from the Local Group. A statistically significant Faraday screen acting on the radio-waves coming from the most distance sources has been found. This analysis supports the existence of a regular magnetic field of $0.3 \mu \mathrm{G}$ in the Local Supercluster.

In the past detection of radio emission in the Coma supercluster has been reported [79. The plane of the Coma supercluster is defined by the Coma cluster and by the Abell cluster 1367 . The observed magnetic field has been extimated to be of the order of $0.5 \mu \mathrm{G}$.

In [80] using numerical simulations the spectrum of protons above $10^{19}$ $\mathrm{eV}$ has been determined under the assumption that the injection spectrum is determined by a discrete set of continuously emitting sources. The sources follow the profile of the local Supercluster. It has been shown that if $|\vec{B}| \leq$ $0.05 \mu \mathrm{G}$ the source distribution assumed in the Supercluster is inconsistent with the observations. On the other hand, if $|\vec{B}| \leq 0.3 \mu \mathrm{G}, 10$ sources in the local Supercluster would lead to spectra consistent with the observations. This analysis seems to fit with [78] and with the general arguments put forward in 55]. In a complementary perspective, the authors of [81] claim to be able to simulate the evolution of large-scale magnetic fields from reasonably high red-shifts. The obtained results, according to [81, correctly reproduce the present large-scale structure of cluster and supercluster magnetic fields both in their observed and unobserved features. 


\section{Globally Neutral Plasmas}

The simplified discussion of the plasma evolution is often related to magnetohydrodynamics (MHD) which is a one-fluid plasma description holding under very specific assumptions. The goal of the first part of the present Section (together with the related Appendix A) will be to present the reduction of the common two-fluids plasma dynamics to the simplified case of one-fluid description provided by MHD.

\subsection{Qualitative aspects of plasma dynamics}

Consider, as a starting point, a globally neutral plasma of electrons and ions (in the simplest case protons) all at the same temperature $T_{0}$ and with mean particle density $n_{0}$. Suppose, for simplicity, local thermodynamical equilibrium ${ }^{11}$. The charge densities will be given, respectively, by

$$
\begin{gathered}
n_{\mathrm{e}}(\phi)=n_{0} e^{e \phi / T_{0}} \simeq\left(1+\frac{e \phi}{T_{0}}\right), \\
n_{\mathrm{p}}(\phi)=n_{0} e^{-e \phi / T_{0}} \simeq\left(1-\frac{e \phi}{T_{0}}\right),
\end{gathered}
$$

where $\phi$ is the electrostatic potential. In Eqs. (4.1) it has been assumed that the plasma is weakly coupled, i.e. $\left|e \phi / T_{0}\right| \ll 1$. A test charge $q_{\mathrm{t}}$ located in the origin will then experience the electrostatic potential following from the Poisson equation,

$$
\nabla^{2} \phi=4 \pi e\left[n_{\mathrm{e}}(\phi)-n_{\mathrm{p}}(\phi)\right]-4 \pi q_{\mathrm{t}} \delta^{(3)}(\vec{x}),
$$

or, using Eqs. (4.1) into Eq. (4.2)

$$
\nabla^{2} \phi-\frac{1}{\lambda_{\mathrm{D}}^{2}} \phi=-4 \pi q_{\mathrm{t}} \delta^{(3)}(\vec{x})
$$

where

$$
\lambda_{\mathrm{D}}=\sqrt{\frac{T_{0}}{8 \pi n_{0} e^{2}}},
$$

is the Debye length. For a test particle the Coulomb potential will then have the usual Coulomb form but it will be suppressed, at large distances by a Yukawa term, i.e. $e^{-r / \lambda_{\mathrm{D}}}$.

\footnotetext{
${ }^{11}$ This assumption is violated in the realistic situations.
} 
In the interstellar medium there are three kinds of regions which are conventionally defined, namely $\mathrm{H}_{2}$ regions (where the Hydrogen is predominantly in molecular form), $\mathrm{H}^{0}$ regions (where Hydrogen is in atomic form) and $\mathrm{H}^{+}$regions (where Hydrogen is ionized). Sometimes $\mathrm{H}^{+}$regions are denoted with $\mathrm{HI}$ and $\mathrm{H}_{2}$ regions with HII. In the $\mathrm{H}^{+}$regions the typical temperature $T_{0}$ is of the order of $10-20 \mathrm{eV}$ while for $n_{0}$ let us take, for instance, $n_{0} \sim 3 \times 10^{-2} \mathrm{~cm}^{-3}$. Then $\lambda_{\mathrm{D}} \sim 30 \mathrm{~km}$.

For $r \gg \lambda_{\mathrm{D}}$ the Coulomb potential is screened by the global effect of the other particles in the plasma. Suppose now that particles exchange momentum through two-body interactions. Their cross section will be of the order of $\alpha_{\mathrm{em}}^{2} / T_{0}^{2}$ and the mean free path will be $\ell_{\mathrm{mfp}} \sim T_{0}^{2} /\left(\alpha_{\mathrm{em}}^{2} n_{0}\right)$, i.e. recalling Eq. (4.4) $\lambda_{\mathrm{D}} \ll \ell_{\mathrm{mfp}}$. This means that the plasma is a weakly collisional system which is, in general, not in local thermodynamical equilibrium. This observation can be made more explicit by defining another important scale, namely the plasma frequency which, in the system under discussion, is given by

$$
\omega_{\mathrm{p}}=\sqrt{\frac{4 \pi n_{0} e^{2}}{m_{\mathrm{e}}}},
$$

where $m_{\mathrm{e}}$ is the electron mass. The plasma frequency is the oscillation frequency of the electrons when they are displaced from their equilibrium configuration in a background of approximately fixed ions. Recalling that $v_{\text {ther }} \simeq \sqrt{T_{0} / m_{\mathrm{e}}}$ is the thermal velocity of the charge carriers, the collision frequency $\omega_{\mathrm{c}} \simeq v_{\text {ther }} / \ell_{\mathrm{mfp}}$ is always much smaller than $\omega_{\mathrm{p}} \simeq v_{\text {ther }} / \lambda_{\mathrm{D}}$. Thus, in the idealized system described so far, the following hierarchy of scales holds:

$$
\lambda_{\mathrm{D}} \ll \ell_{\mathrm{mfp}}, \quad \omega_{\mathrm{c}} \ll \omega_{\mathrm{p}},
$$

which means that before doing one collision the system undergoes many oscillations, or, in other words, that the mean free path is not the shortest scale in the problem. Usually one defines also the plasma parameter $\mathcal{N}=n_{0}^{-1} \lambda_{\mathrm{D}}^{-3}$, i.e. the number of particles in the Debye sphere. In the approximation of weakly coupled plasma $\mathcal{N} \ll 1$ which also imply that the mean kinetic energy of the particles is larger than the mean inter-particle potential, i.e. $|e \phi| \ll T_{0}$ in the language of Eq. (4.1). 


\subsection{Kinetic and fluid descriptions}

From the point of view of the evolution of large-scale magnetic fields, the galaxy is globally neutral system of charged particles with typical rotation period of $3 \times 10^{8}$ yrs. Two complementary descriptions of the plasma can then be adopted. The first possibility is to study the full system of kinetic equations (the Vlasov-Landau description [82, 83, 84, for the one particle distribution function). In the kinetic description the "observables" of the plasma dynamics are related to the various moments of the distribution function. In a complementary perspective, it is also possible to study directly the evolution equations of the various moments of the distribution function (charge and matter densities, momentum transfer etc.). The resulting description is an effective one since not all the moments of the distribution function can be kept. The necessity of a consistent truncation in the hierarchy of the various moments is usually called closure problem. MHD is an effective description which holding for

$$
L \gg \lambda_{\mathrm{D}}, \quad t \gg \omega_{\mathrm{p}}^{-1}
$$

where $L$ and $t$ are the typical length and time scales of the problem. The MHD approximation holds for typical length scales larger than the Debye scale and typical frequencies much smaller than the plasma frequency. In particular, the spectrum of plasma excitations obtained from the full kinetic description matches with the spectrum of MHD excitations but only at sufficiently small frequencies (typically of the order of the ionoacoustic waves) 85, 86.

If the plasma is weakly collisional and out of thermodynamical equilibrium, an approximation where the evolution equations are still tractable is given in terms of the one-particle distribution function $f_{i}(\vec{x}, \vec{v}, t)$ where the subscript denotes the given charge carrier (for instance electrons and ions). The one-particle distribution obeys the Vlasov-Landau equation for each particle species of charge $q_{\mathrm{i}}$ and of mass $m_{\mathrm{i}}$ :

$$
\frac{\partial f_{\mathrm{i}}}{\partial t}+\vec{v} \cdot \vec{\nabla}_{\vec{x}} f_{\mathrm{i}}+\frac{q_{i}}{m_{\mathrm{i}}}(\vec{E}+\vec{v} \times \vec{B}) \cdot \overrightarrow{\nabla_{\vec{v}}} f_{\mathrm{i}}=\left(\frac{\partial f_{\mathrm{i}}}{\partial t}\right)_{\text {coll }} .
$$

The term appearing at the right-hand side of Eq. (4.8) is the binary collision term. In a weakly coupled plasma, the long-range force is given by the Coulomb interaction, while the short-range component arises thanks to binary collisions. In other words the electric and magnetic fields appearing 
in Eq. (4.8) are mean fields obeying mean Maxwell's equations:

$$
\begin{aligned}
& \vec{\nabla} \cdot \vec{E}=4 \pi \sum_{\mathrm{i}} \bar{n}_{\mathrm{i}} q_{\mathrm{i}} \int d^{3} v f_{\mathrm{i}}(\vec{x}, \vec{v}, t), \\
& \vec{\nabla} \times \vec{E}+\frac{\partial \vec{B}}{\partial t}=0, \\
& \vec{\nabla} \times \vec{B}=\frac{\partial \vec{E}}{\partial t}+4 \pi \sum_{\mathrm{i}} \bar{n}_{\mathrm{i}} q_{\mathrm{i}} \int d^{3} v \vec{v} f_{\mathrm{i}}(\vec{x}, \vec{v}, t), \\
& \vec{\nabla} \cdot \vec{B}=0,
\end{aligned}
$$

where $\bar{n}_{\mathrm{i}}$ is the mean particle density. In the context of the kinetic approach the evolution equation should be solved self-consistently with the Maxwell's equations. Clearly, in a system where the density of particles is small the mean field given by the distant particles is more important than the the force produced by the closest particles. In practice the Vlasov-landau system can be linearized around some equilibrium value of the one-particle distribution function and specific examples will be discussed later on.

The one-particle distribution function is not directly observable. The directly observable quantities are the the various moments of the one-particle distribution, for instance, the particle density,

$$
n_{\mathrm{i}}(\vec{x}, t)=\bar{n}_{\mathrm{i}} \int f_{\mathrm{i}}(\vec{x}, \vec{v}, t) d^{3} v,
$$

and the related matter and charge densities for each particle species (i.e. $\rho_{\mathrm{m}, \mathrm{i}}(\vec{x}, t)=m_{\mathrm{i}} n_{\mathrm{i}}(\vec{x}, t)$ and $\left.\rho_{\mathrm{q}, \mathrm{i}}(\vec{x}, t)=q_{\mathrm{i}} n_{\mathrm{i}}(\vec{x}, t)\right)$ or summed over the different species (i.e. $\rho_{\mathrm{m}}=\sum_{\mathrm{i}} \rho_{\mathrm{m}, \mathrm{i}}$ and $\rho_{\mathrm{q}}=\sum_{\mathrm{i}} \rho_{\mathrm{q}, \mathrm{i}}$ ). However, the dynamical informations on the plasma evolution are not only encoded in the moment of order zero but also in the higher moments (the first, the second and even higher).

For some class of problems the kinetic approach is to be preferred. For instance the kinetic approach is mandatory for all the high frequency phenomena (like, for instance, equilibration of charge and current density fluctuations). As far as the low-frequency phenomena are concerned one can imagine to obtain a reduced (fluid-like) description.

\subsection{From one-fluid equations to MHD}

The evolution equations for the one-fluid variables are obtained from the twospecies kinetic description by some algebra which is summarized in the first 
part of Appendix A. The bottom line of the derivation is that the moments of the one-particle distribution function obey a set of partial differential equations whose solutions can be studied in different approximations. One of these approximations is the MHD description. The set of one-fluid equations (derived in Appendix A) can be written as

$$
\begin{aligned}
& \frac{\partial \rho_{\mathrm{m}}}{\partial t}+\vec{\nabla} \cdot\left(\rho_{\mathrm{m}} \vec{v}\right)=0, \\
& \frac{\partial \rho_{\mathrm{q}}}{\partial t}+\vec{\nabla} \cdot \vec{j}=0 . \\
& \rho_{\mathrm{m}}\left[\frac{\partial \vec{v}}{\partial t}+\vec{v} \cdot \vec{\nabla}\right] \vec{v}=\vec{J} \times \vec{B}-\vec{\nabla} P, \\
& \vec{E}+\vec{v} \times \vec{B}=\frac{1}{\sigma} \vec{J}+\frac{1}{e n_{\mathrm{q}}}\left(\vec{J} \times \vec{B}-\vec{\nabla} P_{e}\right) .
\end{aligned}
$$

supplemented by the Maxwell's equations

$$
\begin{array}{ll}
\vec{\nabla} \cdot \vec{E}=4 \pi \rho_{\mathrm{q}}, & \vec{\nabla} \cdot \vec{B}=0 \\
\vec{\nabla} \times \vec{E}+\frac{\partial \vec{B}}{\partial t}=0, & \\
\vec{\nabla} \times \vec{B}=\frac{\partial \vec{E}}{\partial t}+4 \pi \vec{J} &
\end{array}
$$

Eqs. (4.14) and (4.15) are the mass and charge conservation, Eq. (4.16) is the equation for the momentum transfer and Eq. (4.17) is the generalized Ohm law. In Eqs. (4.14) - (4.17) $\vec{v}$ is the one-fluid velocity field, $P$ is the total pressure and $P_{e}$ is the pressure of the electrons.

If the plasma is globally neutral the charge density can be consistently neglected. However, global neutrality is plausible only for typical lengths much larger than $\lambda_{\mathrm{D}}$ where the collective properties of the plasma are the leading dynamical effect. Under this assumption, Eqs. (4.15) and (4.18) imply

$$
\vec{\nabla} \cdot \vec{J}=0, \quad \vec{\nabla} \cdot \vec{E}=0,
$$

which means that MHD currents are, in the first approximation, solenoidal.

The second step to get to a generalized form of the MHD equations is to neglect the terms responsible for the high frequency plasma excitations, like the displacement current in Eqs. (4.20) whose form becomes then

$$
\vec{\nabla} \times \vec{B}=4 \pi \vec{J}
$$


Given the two sets of consistent approximations discussed so far, the form taken by the generalized Ohm law may not be unique and, therefore, different MHD descriptions arise depending upon which term at the right hand side of Eq. (4.17) is consistently neglected. The simplest (but often not realistic) approximation is to neglect all the terms at the right hand side of Eq. (4.17). This is sometimes called ideal MHD approximation. In this case the Ohmic electric field is given by $\vec{E} \simeq-\vec{v} \times \vec{B}$. This ideal description is also called sometimes superconducting MHD approximation since, in this case, the resistive terms in Eq. (4.17), i.e. $\vec{J} / \sigma$ go to zero (and the conductivity $\sigma \rightarrow \infty)$. In the early Universe it is practical to adopt the superconducting approximation (owing to the large value of the conductivity) but it is also very dangerous since all the $\mathcal{O}(1 / \sigma)$ effects are dropped.

A more controllable approximation is the real (or resistive) $M H D$. The resistive approximation is more controllable since the small expansion parameter (i.e. $1 / \sigma$ ) is not zero (like in the ideal case) but it is small and finite. This allows to compute the various quantities to a given order in $1 / \sigma$ even if, for practical purposes, only the first correction is kept. In this framework the Ohmic current is not neglected and the generalized Ohm law takes the form

$$
\vec{J} \simeq \sigma(\vec{E}+\vec{v} \times \vec{B})
$$

Comparing Eq. (4.23) with Eq. (4.17), it is possible to argue that the resistive MHD scheme is inadequate whenever the Hall and thermoelectric terms are cannot be neglected. Defining $\omega_{B}=e B / m$ as the Larmor frequency, the Hall term $\vec{J} \times \vec{B}$ can be neglected provided $L \omega_{\mathrm{p}}^{2} / \omega_{B} v_{0} \gg 1$ where $L$ us the typical size of the system and $v_{0}$ the typical bulk velocity of the plasma. With analogous dimensional arguments it can be argued that the pressure gradient, i.e. the thermoelectric term, can be consistently neglected provided $L v_{0} \omega_{B} m_{e} / T_{e} \gg 1$. Specific examples where the Hall term may become relevant will be discussed in the context of the Biermann battery mechanism.

The set of MHD equations, as it has been presented so far, is not closed if a further relation among the different variables is not specified [87. Typically this is specified through an equation relating pressure and matter density (adiabatic or isothermal closures). Also the incompressible closure (i.e. $\vec{\nabla} \cdot \vec{v}=0$ ) is often justified in the context of the evolution of magnetic fields prior to recombination. It should be stressed that sometimes the adiabatic approximation may lead to paradoxes. It would correspond, in the context of ideal MHD, to infinite electrical conductivity and vanishingly small thermal 
conductivity (i.e. the electrons should be extremely fast not to feel the resistivity and, at the same time, extremely slow not to exchange heat among them).

\subsection{The Alfvén theorems}

The ideal and resistive MHD descriptions are rather useful in order to illustrate some global properties of the evolution of the plasma which are relevant both in the evolution of large-scale magnetic fields prior to recombination and in the discussion of the gravitational collapse in the presence of magnetic fields. These global properties of the plasma evolution go under the name of Alfvén theorems [87] (see also [88]).

In the ideal limit both the magnetic flux and the magnetic helicity are conserved. This means,

$$
\frac{d}{d t} \int_{\Sigma} \vec{B} \cdot d \vec{\Sigma}=-\frac{1}{4 \pi \sigma} \int_{\Sigma} \vec{\nabla} \times \vec{\nabla} \times \vec{B} \cdot d \vec{\Sigma}
$$

where $\Sigma$ is an arbitrary closed surface which moves with the plasma ${ }^{12}$. If $\sigma \rightarrow \infty$ the expression appearing at the right hand side is sub-leading and the magnetic flux lines evolve glued to the plasma element.

The other quantity which is conserved in the superconducting limit is the magnetic helicity

$$
\mathcal{H}_{M}=\int_{V} d^{3} x \vec{A} \cdot \vec{B}
$$

where $\vec{A}$ is the vector potential whose presence may lead to think that the whole expression (4.25) is not gauge-invariant. In fact $\vec{A} \cdot \vec{B}$ is not gauge invariant but, none the less, $\mathcal{H}_{M}$ is gauge-invariant since the integration volume is defined in such a way that the magnetic field $\vec{B}$ is parallel to the surface which bounds $V$ and which we will call $\partial V$. If $\vec{n}$ is the unit vector normal to $\partial V$ then $\vec{B} \cdot \vec{n}=0$ on $\partial V$ and the gauge dependent contribution to the integral appearing in Eq. (4.25) vanishes. In physical terms the integration may always be performed imagining that the space is sliced in flux tubes of the magnetic field. This procedure is correct provided the magnetic flux is, at least approximately, conserved as implied by Eq. (4.24).

The magnetic gyrotropy

$$
\vec{B} \cdot \vec{\nabla} \times \vec{B}
$$

\footnotetext{
${ }^{12}$ Notice that in Ref. 88 Eq. (4.24) has been derived without the $4 \pi$ term at the right hand side because of different conventions.
} 
it is a gauge invariant measure of the diffusion rate of $\mathcal{H}_{M}$. In fact, in the resistive approximation [88]

$$
\frac{d}{d t} \mathcal{H}_{M}=-\frac{1}{4 \pi \sigma} \int_{V} d^{3} x \vec{B} \cdot \vec{\nabla} \times \vec{B}
$$

As in the case of Eq. (4.25), for $\sigma \rightarrow \infty$ the magnetic helicity is approximately constant. The value of the magnetic gyrotropy allows to distinguish different mechanisms for the magnetic field generation. Some mechanisms are able to produce magnetic fields whose flux lines have a non-trivial gyrotropy. The properties of turbulent magnetized plasmas may change depending upon the value of the gyrotropy and of the helicity [87.

The physical interpretation of the flux and magnetic helicity conservation is the following. In MHD the magnetic field has to be always solenoidal (i.e. $\vec{\nabla} \cdot \vec{B}=0$ ). Thus, the magnetic flux conservation implies that, in the superconducting limit (i.e. $\sigma \rightarrow \infty$ ) the magnetic flux lines, closed because of the transverse nature of the field, evolve always glued together with the plasma element. In this approximation, as far as the magnetic field evolution is concerned, the plasma is a collection of (closed) flux tubes. The theorem of flux conservation states then that the energetical properties of large-scale magnetic fields are conserved throughout the plasma evolution.

While the flux conservation concerns the energetical properties of the magnetic flux lines, the magnetic helicity concerns chiefly the topological properties of the magnetic flux lines. If the magnetic field is completely stochastic, the magnetic flux lines will be closed loops evolving independently in the plasma and the helicity will vanish. There could be, however, more complicated topological situations [88] where a single magnetic loop is twisted (like some kind of Möbius stripe) or the case where the magnetic loops are connected like the rings of a chain. In both cases the magnetic helicity will not be zero since it measures, essentially, the number of links and twists in the magnetic flux lines. Furthermore, in the superconducting limit, the helicity will not change throughout the time evolution. The conservation of the magnetic flux and of the magnetic helicity is a consequence of the fact that, in ideal MHD, the Ohmic electric field is always orthogonal both to the bulk velocity field and to the magnetic field. In the resistive MHD approximation this is no longer true.

If the conductivity is very large (but finite), the resistive MHD approximation suggests, on one hand, that the magnetic flux is only approximately conserved. On the other hand the approximate conservation of the magnetic 
helicity implies that the closed magnetic loops may modify their topological structure. The breaking of the flux lines, occurring at finite conductivity, is related to the possibility that bits of magnetic fluxes are ejected from a galaxy into the inter-galactic medium. This phenomenon is called magnetic reconnection and it is the basic mechanism explaining, at least qualitatively, why, during the solar flares, not only charged particles are emitted, but also magnetic fields. In the context of large scale magnetic fields the approximate (or exact) magnetic flux conservation has relevant consequences for the rôle of magnetic fields during gravitational collapse.

\subsection{Magnetic diffusivity equation}

From Eqs. (4.22) and (4.23) the Ohmic electric field can be expressed as

$$
\vec{E}=\frac{1}{4 \pi \sigma} \vec{\nabla} \times \vec{B}-\vec{v} \times \vec{B}
$$

which inserted into Eq. (4.19) leads to the magnetic diffusivity equation

$$
\frac{\partial \vec{B}}{\partial t}=\vec{\nabla} \times(\vec{v} \times \vec{B})+\frac{1}{4 \pi \sigma} \nabla^{2} \vec{B} .
$$

The first term of Eq. (4.29) is the dynamo term. The second term of Eq. (4.29) is the magnetic diffusivity term whose effect is to dissipate the magnetic field. By comparing the left and the right hand side of Eq. (4.29), the typical time scale of resistive phenomena is

$$
t_{\sigma} \simeq 4 \pi \sigma L^{2}
$$

where $L$ is the typical length scale of the magnetic field. In a non-relativistic plasma the conductivity $\sigma$ goes typically as $T^{3 / 2}$ [85]. In the case of planets, like the earth, one can wonder why a sizable magnetic field can still be present. One of the theories is that the dynamo term regenerates continuously the magnetic field which is dissipated by the diffusivity term [20] In the case of the galactic disk the value of the conductivity ${ }^{13}$ is given by $\sigma \simeq 7 \times 10^{-7} \mathrm{~Hz}$. Thus, for $L \simeq \mathrm{kpc} t_{\sigma} \simeq 10^{9}(L / \mathrm{kpc})^{2} \mathrm{sec}$.

\footnotetext{
${ }^{13}$ It is common use in the astrophysical applications to work directly with $\eta=(4 \pi \sigma)^{-1}$. In the case of the galactic disks $\eta=10^{26} \mathrm{~cm}^{2} \mathrm{~Hz}$. The variable $\eta$ denotes, in the present review, the conformal time coordinate.
} 
In Eq. (4.30) the typical time of resistive phenomena has been introduced. Eq. (4.30) can also give the typical resistive length scale once the time-scale of the system is specified. Suppose that the time-scale of the system is given by $t_{U} \sim H_{0}^{-1} \sim 10^{18}$ sec where $H_{0}$ is the present value off the Hubble parameter. Then

$$
L_{\sigma}=\sqrt{\frac{t_{U}}{\sigma}},
$$

leading to $L_{\sigma} \sim \mathrm{AU}$. The scale (4.31) gives then the upper limit on the diffusion scale for a magnetic field whose lifetime is comparable with the age of the Universe at the present epoch. Magnetic fields with typical correlation scale larger than $L_{\sigma}$ are not affected by resistivity. On the other hand, magnetic fields with typical correlation scale $L<L_{\sigma}$ are diffused. The value $L_{\sigma} \sim \mathrm{AU}$ is consistent with the phenomenological evidence that there are no magnetic fields coherent over scales smaller than $10^{-5}$ pc.

The dynamo term may be responsible for the origin of the magnetic field of the galaxy. The galaxy has a typical rotation period of $3 \times 10^{8}$ yrs and comparing this number with the typical age of the galaxy, $\mathcal{O}\left(10^{10} \mathrm{yrs}\right)$, it can be appreciated that the galaxy performed about 30 rotations since the time of the protogalactic collapse.

From Eq. (4.29) the usual structure of the dynamo term may be derived by carefully averaging over the velocity filed according to the procedure of 89 , 90. By assuming that the motion of the fluid is random and with zero mean velocity the average is taken over the ensemble of the possible velocity fields. In more physical terms this averaging procedure of Eq. (4.29) is equivalent to average over scales and times exceeding the characteristic correlation scale and time $\tau_{0}$ of the velocity field. This procedure assumes that the correlation scale of the magnetic field is much bigger than the correlation scale of the velocity field which is required to be divergence-less $(\vec{\nabla} \cdot \vec{v}=0)$. In this approximation the magnetic diffusivity equation can be written as:

$$
\frac{\partial \vec{B}}{\partial t}=\alpha(\vec{\nabla} \times \vec{B})+\frac{1}{4 \pi \sigma} \nabla^{2} \vec{B},
$$

where

$$
\alpha=-\frac{\tau_{0}}{3}\langle\vec{v} \cdot \vec{\nabla} \times \vec{v}\rangle,
$$

is the so-called dynamo term which vanishes in the absence of vorticity. In Eqs. (4.32)-(4.33) $\vec{B}$ is the magnetic field averaged over times longer that $\tau_{0}$ which is the typical correlation time of the velocity field. 
It can be argued that the essential requirement for the consistence of the mentioned averaging procedure is that the turbulent velocity field has to be "globally" non-mirror symmetric [19]. If the system would be, globally, invariant under parity transformations, then, the $\alpha$ term would simply vanish. This observation is related to the turbulent features of cosmic systems. In cosmic turbulence the systems are usually rotating and, moreover, they possess a gradient in the matter density (think, for instance, to the case of the galaxy). It is then plausible that parity is broken at the level of the galaxy since terms like $\vec{\nabla} \rho_{\mathrm{m}} \cdot \vec{\nabla} \times \vec{v}$ are not vanishing [19].

The dynamo term, as it appears in Eq. (4.32), has a simple electrodynamical meaning, namely, it can be interpreted as a mean ohmic current directed along the magnetic field :

$$
\vec{J}=-\alpha \vec{B}
$$

This equation tells us that an ensemble of screw-like vortices with zero mean helicity is able to generate loops in the magnetic flux tubes in a plane orthogonal to the one of the original field. Consider, as a simple application of Eq. (4.32), the case where the magnetic field profile is given by

$$
B_{x}(z, t)=f(t) \sin k z, \quad B_{y}=f(t) \cos k z, \quad B_{z}(k, t)=0 .
$$

For this profile the magnetic gyrotropy is non-vanishing, i.e. $\vec{B} \cdot \vec{\nabla} \times \vec{B}=$ $k f^{2}(t)$. From Eq. (4.32), using Eq. (4.35) $f(t)$ obeys the following equation

$$
\frac{d f}{d t}=\left(k \alpha-\frac{k^{2}}{4 \pi \sigma}\right) f
$$

admits exponentially growing solutions for sufficiently large scales, i.e. $k<$ $4 \pi|\alpha| \sigma$. Notice that in this naive example the $\alpha$ term is assumed to be constant. However, as the amplification proceeds, $\alpha$ may develop a dependence upon $|\vec{B}|^{2}$, i.e. $\alpha \rightarrow \alpha_{0}\left(1-\xi|\vec{B}|^{2}\right) \alpha_{0}\left[1-\xi f^{2}(t)\right]$. In the case of Eq. (4.36) this modification will introduce non-linear terms whose effect will be to stop the growth of the magnetic field. This regime is often called saturation of the dynamo and the non-linear equations appearing in this context are sometimes called Landau equations [19] in analogy with the Landau equations appearing in hydrodynamical turbulence.

In spite of the fact that in the previous example the velocity field has been averaged, its evolution obeys the Navier-Stokes equation

$$
\rho\left[\frac{\partial \vec{v}}{\partial t}+(\vec{v} \cdot \vec{\nabla}) \vec{v}-\nu \nabla^{2} \vec{v}\right]=-\vec{\nabla} p+\vec{J} \times \vec{B}
$$


where $\nu$ is the thermal viscosity coefficient. Since in MHD the matter current is solenoidal (i.e. $\vec{\nabla} \cdot(\rho \vec{v})=0$ ) the incompressible closure $\vec{\nabla} \rho=0$, corresponds to a solenoidal velocity field $\vec{\nabla} \cdot \vec{v}=0$. Recalling Eq. (4.22), the Lorentz force term can be re-expressed through vector identities and Eq. (4.37) becomes

$$
\rho\left[\frac{\partial \vec{v}}{\partial t}+(\vec{v} \cdot \vec{\nabla}) \vec{v}-\nu \nabla^{2} \vec{v}\right]=-\vec{\nabla}\left[p+\frac{|\vec{B}|^{2}}{8 \pi}\right]+(\vec{B} \cdot \vec{\nabla}) \vec{B} .
$$

In typical applications to the evolution of magnetic fields prior to recombination the magnetic pressure term is always smaller than the fluid pressure 14 , i.e. $p \gg|\vec{B}|^{2}$. Furthermore, there are cases where the Lorentz force term can be ignored. This is the so-called force free approximation. Defining the kinetic helicity as $\vec{\omega}=\vec{\nabla} \times \vec{v}$, the magnetic diffusivity and Navier-Stokes equations can be written in a rather simple and symmetric form

$$
\begin{aligned}
& \frac{\partial \vec{B}}{\partial t}=\vec{\nabla} \times(\vec{v} \times \vec{B})+\frac{1}{4 \pi \sigma} \nabla^{2} \vec{B}, \\
& \frac{\partial \vec{\omega}}{\partial t}=\vec{\nabla} \times(\vec{v} \times \vec{\omega})+\nu \nabla^{2} \vec{\omega} .
\end{aligned}
$$

In MHD various dimensionless ratios can be defined. The most frequently used are the magnetic Reynolds number, the kinetic Reynolds number and the Prandtl number:

$$
\begin{aligned}
& \mathrm{R}_{\mathrm{m}}=v L_{B} \sigma, \\
& \mathrm{R}=\frac{v L_{v}}{\nu}, \\
& \operatorname{Pr}=\frac{\mathrm{R}_{\mathrm{m}}}{\mathrm{R}}=\nu \sigma\left(\frac{L_{B}}{L_{v}}\right),
\end{aligned}
$$

where $L_{B}$ and $L_{v}$ are the typical scales of variation of the magnetic and velocity fields. In the absence of pressure and density perturbations the combined system of Eqs. (4.22) and (4.38) can be linearized easily. Using then the incompressible closure the propagating modes are the Alfvén waves whose typical dispersion relation is $\omega^{2}=c_{\mathrm{a}}^{2} k^{2}$ where $c_{\mathrm{a}}=|\vec{B}| / \sqrt{4 \pi \rho}$. Often the

\footnotetext{
${ }^{14}$ Recall that in fusion studies the quantity $\beta=8 \pi|\vec{B}|^{2} / p$ is usually defined. If the plasma is confined, then $\beta$ is of order 1 . On the contrary, if $\beta \gg 1$, as implied by the critical density bound in the early Universe, then the plasma may be compressed at higher temperatures and densities.
} 
Lundqvist number is called, in plasma literature [85, 87] magnetic Reynolds number. This confusion arises from the fact that the Lunqvist number, i.e. $c_{a} L \sigma$, is the magnetic Reynolds number when $v$ coincides with the Alfvén velocity. To have a very large Lundqvist number implies that the the conductivity is very large. In this sense the Lunqvist number characterizes, in fusion theory, the rate of growth of resistive instabilities and it is not necessarily related to the possible occurrence of turbulent dynamics. On the contrary, as large Reynolds numbers are related to the occurrence of hydrodynamical turbulence, large magnetic Reynolds numbers are related to the occurence of MHD turbulence [87].

\subsection{The dynamo mechanism}

According to the naive description of the dynamo instability presented above the origin of large-scale magnetic fields in spiral galaxies can be reduced to the following steps:

- during the 30 rotations performed by the galaxy since the protogalactic collapse, the magnetic field should be amplified by about 30 e-folds;

- if the large scale magnetic field of the galaxy is, today, $\mathcal{O}(\mu \mathrm{G})$ the magnetic field at the onset of galactic rotation might have been even 30 e-folds smaller, i.e. $\mathcal{O}\left(10^{-19} \mathrm{G}\right)$;

- assuming perfect flux freezing during the gravitational collapse of the protogalaxy (i.e. $\sigma \rightarrow \infty$ ) the magnetic field at the onset of gravitational collapse should be $\mathcal{O}\left(10^{-23}\right)$ G over a typical scale of $1 \mathrm{Mpc}$.

This picture is oversimplified and each of the three steps mentioned above will be contrasted with the most recent findings.

The idea that a celestial body may acquire a magnetic field by differential rotation can be traced back to the paper of Larmor of 1919 [91]. One of the early ancestors of the dynamo mechanism, can be traced back to the model of Fermi and Chandrasekar [7, 8, In [7, 8] the attempt was to connect the existence off the galactic magnetic field with the existence of a galactic angular momentum. Later on dynamo theory has been developed in greater detail (see 20]) and its possible application to large-scale magnetic fields has been envisaged.

The standard dynamo theory has been questioned in different ways. Piddington [92, 93] pointed out that small-scale magnetic fields can grow large 
enough (until equipartition is reached) to swamp the dynamo action. The quenching of the dynamo action has been numerically shown by Kulsrud and Anderson [94. More recently, it has been argued that if the large-scale magnetic field reaches the critical value ${ }^{15} R_{\mathrm{m}}^{-1 / 2} v$ the dynamo action could also be quenched [95, 96].

Eq. (4.29) is exact, in the sense that both $\vec{v}$ and $\vec{B}$ contain long and short wavelength modes. The aim of the various attempts of the dynamo theory is to get an equation describing only the "mean value" of the magnetic field. To this end the first step is to separate the exact magnetic and velocity fields as

$$
\begin{aligned}
& \vec{B}=\langle\vec{B}\rangle+\vec{b}, \\
& \vec{v}=\langle\vec{V}\rangle+\vec{V},
\end{aligned}
$$

where $\langle\vec{B}\rangle$ and $\langle\vec{V}\rangle$ are the averages over an ensemble of many realizations of the velocity field $\vec{v}$. In order to derive the standard form of the dynamo equations few important assumptions should be made. These assumptions can be summarized as follows:

- the scale of variation of the turbulent motion $\vec{V}$ should be smaller than the typical scale of variation of $\langle\vec{B}\rangle$. In the galactic problem $\langle\vec{V}\rangle$ is the differential rotation of the galaxy, while $\vec{V}$ is the turbulent motion generated by stars and supernovae. Typically the scale of variation of $\vec{v}$ is less than $100 \mathrm{pc}$ while the interesting scales for $\langle\vec{B}\rangle$ are larger than the kpc;

- the field $\vec{b}$ is such that $|\vec{b}| \ll|\langle\vec{B}\rangle|$.

- it should happen that $\langle\vec{V} \cdot \vec{\nabla} \times \vec{V}\rangle \neq 0$.

- magnetic flux is frozen into the plasma (i.e. magnetic flux is conserved).

From the magnetic diffusivity equation (4.29), and using the listed assumptions, it is possible to derive the typical structure of the dynamo term by carefully averaging over the velocity field according to the procedure outlined in [19, 20, 97]. Inserting Eq. (4.43) into (4.29) and breaking the equation

\footnotetext{
${ }^{15} v$ is the velocity field at the outer scale of turbulence.
} 
into a mean part and a random part, two separate induction equations can be obtained for the mean and random parts of the magnetic field

$$
\begin{aligned}
& \frac{\partial\langle\vec{B}\rangle}{\partial t}=\vec{\nabla} \times(\langle\vec{V}\rangle \times\langle\vec{B}\rangle)+\vec{\nabla} \times\langle\vec{V} \times \vec{b}\rangle, \\
& \frac{\partial \vec{b}}{\partial t}=\vec{\nabla} \times(\vec{V} \times\langle\vec{B}\rangle)+\vec{\nabla} \times(\langle\vec{V}\rangle \times \vec{b}) \\
& +\vec{\nabla} \times(\vec{V} \times \vec{b})-\vec{\nabla} \times\langle\vec{V} \times \vec{b}\rangle,
\end{aligned}
$$

where the (magnetic) diffusivity terms have been neglected. In Eq. (4.44), $\langle\vec{V} \times \vec{b}\rangle$ is called mean field (or turbulent) electromotive force and it is the average of the cross product of the small-scale velocity field $\vec{V}$ and of the small scale magnetic field $\vec{b}$ over a scale much smaller than the scale of $\langle\vec{B}\rangle$ but much larger than the scale of turbulence. Sometimes, the calculation of the effect of $\langle\vec{V} \times \vec{b}\rangle$ is done in the case of incompressible and isotropic turbulence. In this case $\langle\vec{V} \times \vec{b}\rangle=0$. This estimate is, however, not realistic since $\langle\vec{B}\rangle$ is not isotropic. More correctly [97], $\langle\vec{V} \times \vec{b}\rangle$ should be evaluated by using Eq. (4.45) which is usually written in a simplified form

$$
\frac{\partial \vec{b}}{\partial t}=\vec{\nabla} \times(\vec{V} \times\langle\vec{B}\rangle)
$$

where all but the first term of Eq. (4.45) have been neglected. To neglect the term $\vec{\nabla} \times(\langle\vec{V}\rangle \times \vec{b})$ does not pose any problem since it corresponds to choose a reference frame where $\langle\vec{V}\rangle$ is constant. However, the other terms, neglected in Eq. (4.46), are dropped because it is assumed that $|\vec{b}| \ll|\langle\vec{B}\rangle|$. This assumption may not be valid all the time and for all the scales. The validity of Eq. (4.46) seems to require that $\sigma$ is very large so that magnetic diffusivity can keep always $\vec{b}$ small 98 . On the other hand 97] one can argue that $\vec{b}$ is only present over very small scales (smaller than $100 \mathrm{pc}$ ) and in this case the approximate form of eq. (4.46) seems to be more justified.

From Eqs. (4.44)-(4.46) it is possible to get to the final result for the evolution equation of $\langle\vec{B}\rangle$ 97] as it is usally quoted

$$
\frac{\partial\langle\vec{B}\rangle}{\partial t}=\vec{\nabla} \times(\alpha\langle\vec{B}\rangle)+\beta \nabla^{2}\langle\vec{B}\rangle+\vec{\nabla} \times(\langle\vec{V}\rangle \times\langle\vec{B}\rangle),
$$

where

$$
\alpha=-\frac{\tau_{0}}{3}\langle\vec{V} \cdot \vec{\nabla} \times \vec{V}\rangle
$$




$$
\beta=\frac{\tau_{0}}{3}\left\langle\vec{V}^{2}\right\rangle
$$

where $\alpha$ is the dynamo term, $\beta$ is the diffusion term and $\tau_{0}$ is the typical correlation time of the velocity field. The term $\alpha$ is, in general, space-dependent. The standard lore is that the dynamo action stops when the value of the magnetic field reaches the equipartition value (i.e. when the magnetic and kinetic energy of the plasma are comparable). At this point the dynamo "saturates". The mean velocity field can be expressed as $\langle\vec{V}\rangle \simeq \vec{\Omega} \times \vec{r}$ where $|\vec{\Omega}(r)|$ is the angular velocity of differential rotation at the galactocentric radius $r$. In the case of flat rotation curve $|\vec{\Omega}(r)|=\Omega(r) \sim r^{-1}$ which also implies that $\partial|\Omega(r)| / \partial r<1$.

Eq. (4.47) can then be written in terms of the radial and azimuthal components of the mean magnetic field, neglecting, for simplicity, the diffusivity term:

$$
\begin{aligned}
& \frac{\partial\left\langle B_{\phi}\right\rangle}{\partial t}=-r \frac{\partial \Omega}{\partial r}\left\langle B_{r}\right\rangle, \\
& \frac{\partial\left\langle B_{r}\right\rangle}{\partial t}=\alpha \frac{\partial\left\langle B_{\phi}\right\rangle}{\partial r} .
\end{aligned}
$$

The second equation shows that the $\alpha$ effect amplifies the radial component of the large-scale field. Then, through Eq. (4.50) the amplification of the radial component is converted into the amplification of the azimuthal field, this is the $\Omega$ effect.

Usually the picture for the formation of galactic magnetic fields is related to the possibility of implementing the dynamo mechanism. By comparing the rotation period with the age of the galaxy (for a Universe with $\Omega_{\Lambda} \sim 0.7, h \sim$ 0.65 and $\left.\Omega_{\mathrm{m}} \sim 0.3\right)$ the number of rotations performed by the galaxy since its origin is approximately 30. During these 30 rotations the dynamo term of Eq. (4.47) dominates against the magnetic diffusivity. As a consequence an instability develops. This instability can be used in order to drive the magnetic field from some small initial condition up to its observed value. Eq. (4.47) is linear in the mean magnetic field. Hence, initial conditions for the mean magnetic field should be postulated at a given time and over a given scale. This initial mean field, postulated as initial condition of (4.47) is usually called seed.

Most of the work in the context of the dynamo theory focuses on reproducing the correct features of the magnetic field of our galaxy. The achievable amplification produced by the dynamo instability can be at most of $10^{13}$, i.e. 
$e^{30}$. Thus, if the present value of the galactic magnetic field is $10^{-6}$ Gauss, its value right after the gravitational collapse of the protogalaxy might have been as small as $10^{-19}$ Gauss over a typical scale of $30-100 \mathrm{kpc}$.

There is a simple way to relate the value of the magnetic fields right after gravitational collapse to the value of the magnetic field right before gravitational collapse. Since the gravitational collapse occurs at high conductivity the magnetic flux and the magnetic helicity are both conserved. Right before the formation of the galaxy a patch of matter of roughly 1 Mpc collapses by gravitational instability. Right before the collapse the mean energy density of the patch, stored in matter, is of the order of the critical density of the Universe. Right after collapse the mean matter density of the protogalaxy is, approximately, six orders of magnitude larger than the critical density.

Since the physical size of the patch decreases from $1 \mathrm{Mpc}$ to $30 \mathrm{kpc}$ the magnetic field increases, because of flux conservation, of a factor $\left(\rho_{\mathrm{a}} / \rho_{\mathrm{b}}\right)^{2 / 3} \sim$ $10^{4}$ where $\rho_{\mathrm{a}}$ and $\rho_{\mathrm{b}}$ are, respectively the energy densities right after and right before gravitational collapse. The correct initial condition in order to turn on the dynamo instability would be $|\vec{B}| \sim 10^{-23}$ Gauss over a scale of $1 \mathrm{Mpc}$, right before gravitational collapse.

This last estimate is rather generous and has been presented just in order to make contact with several papers (concerned with the origin of large scale magnetic fields) using such an estimate. The estimates presented in the last paragraph are based on the (rather questionable) assumption that the amplification occurs over thirty e-folds while the magnetic flux is completely frozen in. In the real situation, the achievable amplification is much smaller. Typically a good seed would not be $10^{-19} \mathrm{G}$ after collapse (as we assumed for the simplicity of the discussion) but rather [97]

$$
|\vec{B}| \geq 10^{-13} \mathrm{G} .
$$

The possible applications of dynamo mechanism to clusters is still under debate and it seems more problematic [73, 74]. The typical scale of the gravitational collapse of a cluster is larger (roughly by one order of magnitude) than the scale of gravitational collapse of the protogalaxy. Furthermore, the mean mass density within the Abell radius $\left(\simeq 1.5 h^{-1} \mathrm{Mpc}\right.$ ) is roughly $10^{3}$ larger than the critical density. Consequently, clusters rotate much less than galaxies. Recall that clusters are formed from peaks in the density field. The present overdensity of clusters is of the order of $10^{3}$. Thus, in order to get the intra-cluster magnetic field, one could think that magnetic flux is exactly 
conserved and, then, from an intergalactic magnetic field $|\vec{B}|>10^{-9} \mathrm{G}$ an intra cluster magnetic field $|\vec{B}|>10^{-7} \mathrm{G}$ can be generated. This simple estimate shows why it is rather important to improve the accuracy of magnetic field measurements in the intra-cluster medium discussed in Section 3: the change of a single order of magnitude in the estimated magnetic field may imply rather different conclusions for its origin. Recent numerical simulations seem to support the view that cluster magnetic fields are entirely primordial 99].

\section{Magnetic Fields in Curved Backgrounds}

As the temperature increases above $1 \mathrm{MeV}$, a relativistic plasma becomes almost a perfect conductor. There are two complementary effects associated with large-scale magnetic fields in curved backgrounds:

- large-scale magnetic fields may evolve over a rigid background spacetime determined, for instance, by the dynamics of barotropic fluids and, in this case the energy density of the magnetic field must always be smaller than the energy density of the fluid sources;

- magnetic fields may be so intense to modify the structure of the spacetime and, in this case, their energy density is comparable with the energy density of the other sources of the geometry.

The first effect will be examined in the present Section, while the discussion of the second effect, which is more speculative, will be confined to Section 9 .

\subsection{The standard cosmological model}

The standard model of cosmological evolution rests on three important assumptions [100. The first assumption is that over very large length scales (greater than $50 \mathrm{Mpc}$ ) the Universe is described by a homogeneous and isotropic Friedmann-Robertson-Walker (FRW) line element:

$$
d s^{2}=G_{\mu \nu} d x^{\mu} d x^{\nu}=a^{2}(\eta)\left[d \eta^{2}-d \vec{x}^{2}\right]
$$

where $a(\eta)$ is the scale factor and $\eta$ the conformal-time coordinate (notice that Eq. (5.1) has been written, for simplicity, in the conformally flat case). The second hypothesis is that the sources of the evolution of the background 
geometry are perfect fluid sources. As a consequence the entropy of the sources is constant. The third and final hypothesis is that the dynamics of the sources and of the geometry is dictated by the general relativistic FRW equations ${ }^{16}$ :

$$
\begin{aligned}
& M_{\mathrm{P}}^{2} \mathcal{H}^{2}=\frac{a^{2}}{3} \rho, \\
& M_{\mathrm{P}}^{2}\left(\mathcal{H}^{2}-\mathcal{H}^{\prime}\right)=\frac{a^{2}}{2}(\rho+p), \\
& \rho^{\prime}+3 \mathcal{H}(\rho+p)=0,
\end{aligned}
$$

where $\mathcal{H}=(\ln a)^{\prime}$ and the prime ${ }^{17}$ denotes the derivation with respect to $\eta$. Recall also, for notational convenience, that $a H=\mathcal{H}$ where $H=\dot{a} / a$ is the conventional Hubble parameter. The various tests of the standard cosmological model are well known [101, 102]. Probably one of the most stringent one comes from the possible distortions, in the Rayleigh-Jeans region, of the CMB spectrum. The absence of these distortions clearly rules out steadystate cosmological models. In the standard cosmological model one usually defines the proper distance of the event horizon at the time $t_{1}$

$$
d_{\mathrm{e}}=a\left(t_{1}\right) \int_{t_{1}}^{t_{\max }} \frac{d t}{a(t)}
$$

this distance represents the maximal extension of the region over which causal connection is possible. Furthermore, the proper distance of the particle horizon can also be defined:

$$
d_{\mathrm{p}}\left(t_{2}\right)=a\left(t_{2}\right) \int_{t_{\min }}^{t_{2}} \frac{d t}{a(t)}
$$

If the scale factor is parametrized as $a(t) \sim t^{\alpha}$, for $0<\alpha<1$ the Universe experiences a decelerated expansion, i.e. $\dot{a}>0$ and $\ddot{a}<0$ while the curvature scale decreases, i.e. $\dot{H}<0$. This is the peculiar behaviour when the fluid sources are dominated either by dust $(p=0)$ or by radiation $(p=\rho / 3)$. In the case of the standard model, the particle horizon increases linearly in cosmic time (therefore faster than the scale factor). This implies that the $\mathrm{CMB}$ radiation, today observed with a temperature of $2.7 \mathrm{~K}$ over the whole

\footnotetext{
${ }^{16}$ Units $M_{\mathrm{P}}=(8 \pi G)^{-1 / 2}=1.72 \times 10^{18} \mathrm{GeV}$ will be used.

${ }^{17}$ The overdot will usually denote derivation with respect to the cosmic time coordinate $t$ related to conformal time as $d t=a(\eta) d \eta$.
} 
present horizon has been emitted from space-time regions which were not in causal contact. This problem is known as the horizon problem of the standard cosmological model. The other problem of the standard model is related to the fact that today the intrinsic (spatial) curvature $k / a^{2}$ is smaller than the extrinsic curvature, i.e. $H^{2}$. Recalling that $k /\left(a^{2} H^{2}\right)=k / \dot{a}^{2}$ it is clear that if $\ddot{a}<0,1 / \dot{a}^{2}$ increases so that the intrinsic curvature could be, today, arbitrarily large. The third problem of the standard cosmological model is related to the generation of the large entropy of the present Universe. The solution of the kinematical problems of the standard model is usually discussed in the framework of a phase of accelerated expansion [103], i.e. $\ddot{a}>0$ and $\dot{a}>0$. In the case of inflationary dynamics the extension of the causally connected regions grows as the scale factor and hence faster than in the decelerated phase. This solves the horizon problem. Furthermore, during inflation the contribution of the spatial curvature becomes very small. The way inflation solves the curvature problem is by producing a very tiny spatial curvature at the onset of the radiation epoch taking place right after inflation. The spatial curvature can well grow during the decelerated phase of expansion but is will be always subleading provided inflation lasted for sufficiently long time. In fact, the minimal requirement in order to solve these problems is that inflation lasts, at least, 60-efolds. The final quantity which has to be introduced is the Hubble radius $H^{-1}(t)$. This quantity is local in time, however, a sloppy nomenclature often exchanges the Hubble radius with the horizon. Since this terminology is rather common, it will also be used here. In the following applications it will be relevant to recall some of the useful thermodynamical relations. In particular, in radiation dominated Universe, the relation between the Hubble parameter and the temperature is given by

$$
\begin{aligned}
& H=1.08 \times\left(\frac{g_{*}}{10.75}\right)^{1 / 2} \frac{T^{2}}{M_{\mathrm{P}}}, \\
& H^{-1}=9 \times 10^{4}\left(\frac{g_{*}}{10.75}\right)^{-1 / 2}\left(\frac{\mathrm{GeV}}{T}\right)^{2} \mathrm{~cm},
\end{aligned}
$$

where $g_{*}$ is the effective number of relativistic degrees of freedom at the corresponding temperature. Eq. (5.2), implying $H^{2} M_{\mathrm{P}}^{2}=\rho / 3$, has been used in Eq. (5.7) together with the known relations valid in a radiation dominated background

$$
\rho(T)=\frac{\pi^{2}}{30} g_{*} T^{4}, \quad n(T)=\frac{\zeta(3)}{\pi^{2}} g_{*} T^{3}
$$


where $\zeta(3) \simeq 1.2$. In Eq. (5.8) the thermodynamical relation for the number density $n(T)$ has been also introduced for future convenience.

\subsubsection{Inflationary dynamics and its extensions}

The inflationary dynamics can be realized in different ways. Conventional inflationary models are based either on one single inflaton field 104, 105, 106, 107] or on various fields [108 (see 109] for a review). Furthermore, in the context of single-field inflationary models one oughts to distinguish between small-field models [106] (like in the so-called new-inflationary models) and large-field models [107] (like in the case of chaotic models).

In spite of their various quantitative differences, conventional inflationary models are based on the idea that during the phase of accelerated expansion the curvature scale is approximately constant (or slightly decreasing). After inflation, the radiation dominated phase starts. It is sometimes useful for numerical estimates to assume that radiation suddenly dominates at the end of inflation. In this case the scale factor can be written as

$$
\begin{aligned}
& a_{i}(\eta)=\left(-\frac{\eta}{\eta_{1}}\right)^{-\alpha}, \quad \eta<-\eta_{1}, \\
& a_{r}(\eta)=\frac{\alpha \eta+(\alpha+1) \eta_{1}}{\eta_{1}}, \quad \eta \geq-\eta_{1},
\end{aligned}
$$

where $\alpha$ is some effective exponent parameterizing the dynamics of the primordial phase of the Universe. Notice that if $\alpha=1$ we have that the primordial phase coincides with a de Sitter inflationary epoch. The case $\alpha=1$ is not completely realistic since it corresponds to the case where the energymomentum tensor is simply given by a (constant) cosmological term. In this case the scalar fluctuations of the geometry are not amplified and the large-scale angular anisotropy in the CMB would not be reproduced. The idea is then to discuss more realistic energy-momentum tensors leading to a dynamical behaviour close to the one of pure de Sitter space, hence the name quasi-de Sitter space-times. Quasi-de Sitter dynamics can be realized in different ways. One possibility is to demand that the inflaton slowly rolls down from its potential obeying the approximate equations

$$
\begin{aligned}
& M_{\mathrm{P}}^{2} H^{2} \simeq \frac{V(\varphi)}{3}, \\
& 3 H \dot{\varphi} \simeq-\frac{\partial V}{\partial \varphi},
\end{aligned}
$$


valid provided $\epsilon_{1}=-\dot{H} / H^{2} \ll 1$ and $\epsilon_{2}=\ddot{\varphi} /(H \dot{\varphi}) \ll 1$. There also exist exact inflationary solutions like the power-law solutions obtainable in the case of exponential potentials:

$$
\begin{aligned}
& V=V_{0} e^{-\sqrt{\frac{2}{p}} \frac{\varphi}{M_{\mathrm{P}}}}, \quad \dot{\varphi}=\frac{\sqrt{2 p} M_{\mathrm{P}}}{t}, \\
& a(t) \simeq t^{p} .
\end{aligned}
$$

Since, from the exact equations, $2 M_{\mathrm{P}}^{2} \dot{H}=-\dot{\varphi}^{2}$ the two slow-roll parameters can also be written as

$$
\epsilon_{1}=\frac{M_{\mathrm{P}}^{2}}{2}\left(\frac{\partial \ln V}{\partial \varphi}\right)^{2}, \quad \epsilon_{2}=-\frac{M_{\mathrm{P}}^{2}}{2}\left(\frac{\partial \ln V}{\partial \varphi}\right)^{2}+\frac{M_{\mathrm{P}}^{2}}{V} \frac{\partial^{2} V}{\partial \varphi^{2}} .
$$

In the case of the exponential potential (5.11) the slow-roll parameters are all equal, $\epsilon_{1}=\epsilon_{2}=1 / p$. Typical potentials leading to the usual inflationary dynamics are power-law potentials of the type $V(\varphi) \simeq \varphi^{n}$, exponential potentials, trigonometric potentials and nearly any potential satisfying, in some region of the parameter space, the slow-roll conditions.

Inflation can also be realized in the case when the curvature scale is increasing, i.e. $\dot{H}>0$ and $\ddot{a}>0$. This is the case of superinflationary dynamics. For instance the propagation of fundamental strings in curved backgrounds [110, 111] may lead to superinflationary solutions [113, 114]. A particularly simple case of superinflationary solutions arises in the case when internal dimensions are present.

Consider a homogeneous and anisotropic manifold whose line element can be written as

$$
\begin{gathered}
d s^{2}=G_{\mu \nu} d x^{\mu} d x^{\nu}=a^{2}(\eta)\left[d \eta^{2}-\gamma_{i j} d x^{i} d x^{j}\right]-b^{2}(\eta) \gamma_{a b} d y^{a} d y^{b} \\
\mu, \nu=0, \ldots, D-1=d+n, \quad i, j=1, \ldots, d, \quad a, b=d+1, \ldots, d+n
\end{gathered}
$$

[ $\eta$ is the conformal time coordinate related, as usual to the cosmic time $t=$ $\int a(\eta) d \eta ; \gamma_{i j}(x), \gamma_{a b}(y)$ are the metric tensors of two maximally symmetric Euclidean manifolds parameterized, respectively, by the "internal" and the "external" coordinates $\left\{x^{i}\right\}$ and $\left\{y^{a}\right\}$ ]. The metric of Eq. (5.13) describes the situation in which the $d$ external dimensions (evolving with scale factor $a(\eta)$ ) and the $n$ internal ones (evolving with scale factor $b(\eta)$ ) are dynamically decoupled from each other [115]. 
A model of background evolution can be generically written as

$$
\begin{array}{lr}
a(\eta)=a_{1}\left(-\frac{\eta}{\eta_{1}}\right)^{\sigma}, \quad b(\eta)=b_{1}\left(-\frac{\eta}{\eta_{1}}\right)^{\lambda}, & \eta<-\eta_{1}, \\
a(\eta)=a_{1}\left(\frac{\eta+2 \eta_{1}}{\eta_{1}}\right), \quad b(\eta)=b_{1}, & -\eta_{1} \leq \eta \leq \eta_{2}, \\
a(\eta)=a_{1} \frac{\left(\eta+\eta_{2}+4 \eta_{1}\right)^{2}}{4 \eta_{1}\left(\eta_{2}+2 \eta_{1}\right)}, \quad b(\eta)=b_{1}, & \eta>\eta_{2} .
\end{array}
$$

In the parameterization of Eq. (5.14) the internal dimensions grow (in conformal time) for $\lambda<0$ and they shrink for $\lambda>0{ }^{18}$.

Superinflationary solutions are also common in the context of the lowenergy string effective action [119, 120, 121]. In critical superstring theory the electromagnetic field $F_{\mu \nu}$ is coupled not only to the metric $\left(g_{\mu \nu}\right)$, but also to the dilaton background $(\varphi)$. In the low energy limit such interaction is represented by the string effective action [119, 120, 121, which reads, after reduction from ten to four expanding dimensions,

$$
\begin{aligned}
& S=-\int d^{4} x \sqrt{-g} e^{-\varphi}\left[R+\partial_{\mu} \varphi \partial^{\mu} \varphi-\frac{1}{2} e^{2 \varphi} \partial_{\mu} \sigma \partial^{\mu} \sigma\right. \\
& \left.+\frac{1}{4} F_{\mu \nu} F^{\mu \nu}-\frac{1}{8} e^{\varphi} \sigma F_{\mu \nu} \tilde{F}^{\mu \nu}\right]
\end{aligned}
$$

were $\varphi=\phi-\ln V_{6} \equiv \ln \left(g^{2}\right)$ controls the tree-level four-dimensional gauge coupling ( $\phi$ being the ten-dimensional dilaton field, and $V_{6}$ the volume of the six-dimensional compact internal space). The field $\sigma$ is the Kalb-Ramond axion whose pseudoscalar coupling to the gauge fields may also be interesting.

In the inflationary models based on the above effective action [122, 123] the dilaton background is not at all constant, but undergoes an accelerated evolution from the string perturbative vacuum $(\varphi=-\infty)$ towards the strong coupling regime, where it is expected to remain frozen at its present value. The peculiar feature of this string cosmological scenario (sometimes called pre-big bang [123]) is that not only the curvature evolves but also the gauge coupling. Suppose, for the moment that the gauge fields are set to zero. The

\footnotetext{
${ }^{18}$ To assume that the internal dimensions are constant during the radiation and matter dominated epoch is not strictly necessary. If the internal dimensions have a time variation during the radiation phase we must anyway impose the BBN bounds on their variation [16, 117, 118. The tiny variation allowed by BBN implies that $b(\eta)$ must be effectively constant for practical purposes.
} 
phase of growing curvature and dilaton coupling $(\dot{H}>0, \dot{\varphi}>0)$, driven by the kinetic energy of the dilaton field, is correctly described in terms of the lowest order string effective action only up to the conformal time $\eta=\eta_{s}$ at which the curvature reaches the string scale $H_{s}=\lambda_{s}^{-1}\left(\lambda_{s} \equiv \sqrt{\alpha^{\prime}}\right.$ is the fundamental length of string theory). A first important parameter of this cosmological model is thus the value $\varphi_{s}$ attained by the dilaton at $\eta=\eta_{s}$. Provided such value is sufficiently negative (i.e. provided the coupling $g=e^{\varphi / 2}$ is sufficiently small to be still in the perturbative region at $\eta=\eta_{s}$ ), it is also arbitrary, since there is no perturbative potential to break invariance under shifts of $\varphi$. For $\eta>\eta_{s}$ high-derivatives terms (higher orders in $\alpha^{\prime}$ ) become important in the string effective action, and the background enters a genuinely "stringy" phase of unknown duration. An assumption of string cosmology is that the stringy phase eventually ends at some conformal time $\eta_{1}$ in the strong coupling regime. At this time the dilaton, feeling a non-trivial potential, freezes to its present constant value $\varphi=\varphi_{1}$ and the standard radiation-dominated era starts. The total duration $\eta_{1} / \eta_{s}$, or the total redshift $z_{s}$ encountered during the stringy epoch (i.e. between $\eta_{s}$ and $\eta_{1}$ ), will be the second crucial parameter besides $\varphi_{s}$ entering our discussion. For the purpose of this paper, two parameters are enough to specify completely our model of background, if we accept that during the string phase the curvature stays controlled by the string scale, that is $H \simeq g M_{\mathrm{P}} \simeq \lambda_{s}^{-1}\left(M_{p}\right.$ is the Planck mass) for $\eta_{s}<\eta<\eta_{1}$.

During the string era $\dot{\varphi}$ and $H$ are approximately constant, while, during the dilaton-driven epoch

$$
a=(-t)^{\alpha}, \quad \alpha=-\frac{1}{\sqrt{3}} \sqrt{1-\Sigma},
$$

Here $\Sigma \equiv \sum_{i} \beta_{i}^{2}$ represents the possible effect of internal dimensions whose radii $b_{i}$ shrink like $(-t)^{\beta_{i}}$ for $t \rightarrow 0_{-}$(for the sake of definiteness we show in the figure the case $\Sigma=0$ ). The shape of the coupling curve corresponds to the fact that the dilaton is constant during the radiation era, that $\dot{\varphi}$ is approximately constant during the string era, and that it evolves like

$$
g(\eta)=e^{\varphi / 2}=a^{\lambda}, \quad \lambda=\frac{1}{2}\left(3+\frac{\sqrt{3}}{\sqrt{1-\Sigma}}\right),
$$

during the dilaton-driven era.

An interesting possibility, in the pre-big bang context is that the exit to the phase of decelerated expansion and decreasing curvature takes place 
without any string tension correction. Recently a model of this kind has been proposed 124. The idea consists in adding a non-local dilaton potential which is invariant under scale factor duality. The evolution equations of the metric and of the dilaton will then become, in 4 space-time dimensions,

$$
\begin{aligned}
& \dot{\bar{\varphi}}^{2}-3 H^{2}-V=0, \\
& \dot{H}-H \dot{\varphi}=0 \\
& 2 \ddot{\bar{\varphi}}-\dot{\bar{\varphi}}^{2}-3 H^{2}+V-\frac{\partial V}{\partial \bar{\varphi}}=0
\end{aligned}
$$

where $\bar{\varphi}=\varphi-3 \log a$ is the shifted dilaton. A particular solution to these equations will be given by

$$
\begin{aligned}
& V(\bar{\varphi})=-V_{0} e^{4 \bar{\varphi}}, \\
& a(t)=a_{0}\left[\tau+\sqrt{\tau^{2}+1}\right]^{1 / \sqrt{3}}, \\
& \bar{\varphi}=-\frac{1}{2} \log \left(1+\tau^{2}\right)+\varphi_{0},
\end{aligned}
$$

where

$$
\tau=\frac{t}{t_{0}}, \quad t_{0}=\frac{e^{-2 \varphi_{0}}}{\sqrt{V_{0}}} .
$$

This solution interpolates smoothly between two self-dual solutions. For $t \rightarrow-\infty$ the background superinflates while for $t \rightarrow+\infty$ the decelerated FRW limit is recovered.

\subsection{Gauge fields in FRW Universes}

In a FRW metric of the type (5.1) the evolution equations of the gauge fields are invariant under Weyl rescaling of the four-dimensional metric. This property is often named conformal invariance. Consider, for simplicity, the evolution equation of sourceless Maxwell fields:

$$
\nabla_{\mu} F^{\mu \nu} \equiv \frac{1}{\sqrt{-G}} \partial_{\mu}\left[\sqrt{-G} F^{\mu \nu}\right]=0
$$

Since the metric (5.1) is conformally flat, the time dependence arising in Eq. (5.23) from $\sqrt{-G}$, is always compensated by the controvariant indices of the fields strength. Maxwell fields in conformally flat metrics obey the 
same equations obtained in the case of Minkowski space-time provided the conformal time coordinate is used and provided the fields are appropriately rescaled. This property holds not only for Maxwell fields but also for chiral fermions in FRW backgrounds.

Using Weyl invariance, the generalization of MHD equations to the case of conformally flat FRW space-times can then be easily obtained:

$$
\begin{aligned}
& \frac{\partial \overrightarrow{\mathcal{B}}}{\partial \eta}+\vec{\nabla} \times \overrightarrow{\mathcal{E}}=0 \\
& \vec{\nabla} \cdot \overrightarrow{\mathcal{E}}=0, \quad \vec{\nabla} \cdot \overrightarrow{\mathcal{B}}=0 \\
& \vec{\nabla} \times \overrightarrow{\mathcal{B}}=4 \pi \overrightarrow{\mathcal{J}}+\frac{\partial \overrightarrow{\mathcal{E}}}{\partial \eta} \\
& \overrightarrow{\mathcal{J}}=\sigma(\overrightarrow{\mathcal{E}}+\vec{v} \times \overrightarrow{\mathcal{B}})
\end{aligned}
$$

where

$$
\overrightarrow{\mathcal{B}}=a^{2} \vec{B}, \quad \overrightarrow{\mathcal{E}}=a^{2} \vec{E}, \quad \overrightarrow{\mathcal{J}}=a^{3} \vec{J}, \quad \sigma=\sigma_{c} a,
$$

are the curved space fields expressed as a function of the corresponding flat space quantities.

In the case of a radiation dominated background, the Navier-Stokes equation takes the form

$$
[(\bar{p}+\bar{\rho}) \vec{v}]^{\prime}+\vec{v} \cdot \vec{\nabla}[(\bar{p}+\bar{\rho}) \vec{v}]=-\vec{\nabla}\left[\bar{p}+\frac{\left.|\overrightarrow{\mathcal{B}}|^{2}\right]}{8 \pi}\right]+\frac{[\overrightarrow{\mathcal{B}} \cdot \vec{\nabla}] \overrightarrow{\mathcal{B}}}{4 \pi}+(\bar{p}+\bar{\rho}) \nu \nabla^{2} \vec{v}
$$

where the incompressible closure, i.e. $\vec{\nabla} \cdot \vec{v}=0$, has been already adopted and where the vector identity $\vec{\nabla} \times \overrightarrow{\mathcal{B}} \times \overrightarrow{\mathcal{B}}=-\frac{1}{2} \vec{\nabla}|\overrightarrow{\mathcal{B}}|^{2}+[\overrightarrow{\mathcal{B}} \cdot \vec{\nabla}] \overrightarrow{\mathcal{B}}$ has been used. In Eq. (5.29) $\bar{p}=a^{4} p$ and $\bar{\rho}=a^{4} \rho$. In terms of the rescaled pressure and energy densities the continuity equation becomes

$$
\frac{\partial \bar{\rho}}{\partial \eta}+(3 \gamma-1) \mathcal{H} \bar{\rho}+(\gamma+1) \vec{\nabla} \cdot[\bar{\rho} \vec{v}]=0,
$$

where a barotropic equation of state has been assumed for the background fluid, i.e. $\bar{p}=\gamma \bar{\rho}$. The energy density is homogeneous and hence the last term in Eq. (5.30) can be consistently dropped within the incompressible closure. However, one may ought to linearize Eq. (5.30) and this is why the last term may be kept for future considerations. If $\gamma=1 / 3$, then Eq. (5.30) implies $\bar{\rho}^{\prime}=0$. For generic barotropic index Eq. (5.29) becomes

$$
\frac{\partial \vec{v}}{\partial \eta}+(1-3 \gamma) \mathcal{H} \vec{v}=\frac{[\overrightarrow{\mathcal{B}} \cdot \vec{\nabla}] \overrightarrow{\mathcal{B}}}{4 \pi(\gamma+1) \bar{\rho}}+\nu \nabla^{2} \vec{v}
$$


where the magnetic pressure term has been dropped since it is negligible with respect to $\bar{p}$.

Recalling that for $L \gg L_{\sigma}$ the magnetic diffusivity equation can be written, using vector identities, as

$$
\frac{\partial \overrightarrow{\mathcal{B}}}{\partial \eta}+[\vec{v} \cdot \vec{\nabla}] \overrightarrow{\mathcal{B}}=[\overrightarrow{\mathcal{B}} \cdot \vec{\nabla}] \vec{v}+\frac{1}{4 \pi \sigma} \nabla^{2} \overrightarrow{\mathcal{B}}
$$

Eqs. (5.24) -(5.29) form a closed system sharing analogies with their flat space counterpart. Thanks to conformal invariance, various solutions can be lifted from flat to curved space like, for instance [87], the following fully nonlinear solution of Eqs. (5.32) and (5.29)

$$
\vec{v}= \pm \frac{\overrightarrow{\mathcal{B}}}{\sqrt{\bar{\rho}+\bar{p}}} \sim \pm \frac{\overrightarrow{\mathcal{B}}}{\sqrt{\bar{\rho}}}
$$

valid if thermal and magnetic diffusivities are neglected and if $\bar{p} \gg|\overrightarrow{\mathcal{B}}|^{2}$.

Eqs. (5.24) - (5.29) present also some differences with respect to the flat space case: since the Universe is expanding, the relative balance between diffusion scales may change as time goes by. All the propagation of MHD excitations can be then generalized to the curved-space case. In various papers [125, 126, 127, 127. this program has been achieved (see also [129, 130] for earlier attempts). In [88] the evolution of magnetic fields with non-vanishing magnetic helicity has been studied using the resistive MHD approximation in curved space. In 131 the evolution of non-linear Alfvén waves with the purpose of deriving possible observable effects 132 .

In order to analyze the high-frequency spectrum of plasma excitations, the Vlasov-Landau approach can be generalized to curved spaces 133. There are two options in order to achieve this goal. The first option is to assume that the magnetic field breaks the isotropy of space-time. This approach leads to the study of plasma effects in a magnetic field in full analogy with what is done in terrestrial tokamaks. The second choice is to discuss magnetic fields which are stochastic and which do not break the isotropy. Later in the present review the effects of magnetic fields breaking the isotropy of space-time will be considered but, for the moment, the attention will be concentrated on the second approach.

A typical problem which should be discussed within the kinetic approach is the relaxation of charge and current density fluctuations in a relativistic 
plasma [134]. The problem is then to compute what magnetic field is induced from charge and current density fluctuations.

Consider an equilibrium homogeneous and isotropic conducting plasma, characterized by a distribution function

$$
f_{0}(p)=\frac{n_{q}}{8 \pi T^{3}} e^{-\frac{p}{T}},
$$

common for both positively and negatively charged ultrarelativistic particles (for example, electrons and positrons). The normalization is chosen in such a way that $\int d^{3} p f_{0}(p)=n_{q}$. Within the kinetic approach, the initial charge and current density fluctuations are related to the perturbations of the initial value of the distribution function around the equilibrium distribution (5.34), i.e

$$
\begin{aligned}
& f_{+}(\vec{x}, \vec{p}, \eta)=f_{0}(p)+\delta f_{+}(\vec{x}, \vec{p}, \eta), \\
& f_{-}(\vec{x}, \vec{p}, \eta)=f_{0}(p)+\delta f_{-}(\vec{x}, \vec{p}, \eta),
\end{aligned}
$$

where + refers to positrons and - to electrons, and $\vec{p}$ is the conformal momentum. The Vlasov equation defining the curved-space evolution of the perturbed distributions can be written as

$$
\begin{aligned}
& \frac{\partial f_{+}}{\partial \eta}+\vec{v} \cdot \frac{\partial f_{+}}{\partial \vec{x}}+e(\overrightarrow{\mathcal{E}}+\vec{v} \times \overrightarrow{\mathcal{B}}) \cdot \frac{\partial f_{+}}{\partial \vec{p}}=\left(\frac{\partial f_{+}}{\partial \eta}\right)_{\text {coll }}, \\
& \frac{\partial f_{-}}{\partial \eta}+\vec{v} \cdot \frac{\partial f_{-}}{\partial \vec{x}}-e(\overrightarrow{\mathcal{E}}+\vec{v} \times \overrightarrow{\mathcal{B}}) \cdot \frac{\partial f_{-}}{\partial \vec{p}}=\left(\frac{\partial f_{-}}{\partial \eta}\right)_{\text {coll }},
\end{aligned}
$$

where the two terms appearing at the right hand side of each equation are the collision terms. This system of equation represents the curved space extension of the Vlasov-Landau approach to plasma fluctuations [82, 83. All particle number densities here are related to the comoving volume.

Notice that, in general $\vec{v}=\vec{p} / \sqrt{\vec{p}^{2}+m_{e}^{2} a^{2}}$. In the ultra-relativistic limit $\vec{v}=\vec{p} /|\vec{p}|$ and the Vlasov equations are conformally invariant. This implies that, provided we use conformal coordinates and rescaled gauge fields, the system of equations which we would have in flat space [84] looks exactly the same as the one we are discussing in a curved FRW (spatially flat) background 133 .

The evolution equations of the gauge fields coupled to the two Vlasov equations can be written as

$$
\vec{\nabla} \cdot \overrightarrow{\mathcal{E}}=4 \pi e \int d^{3} p\left[f_{+}(\vec{x}, \vec{p}, \eta)-f_{-}(\vec{x}, \vec{p}, \eta)\right],
$$




$$
\begin{aligned}
& \vec{\nabla} \times \overrightarrow{\mathcal{E}}+\overrightarrow{B^{\prime}}=0 \\
& \vec{\nabla} \cdot \overrightarrow{\mathcal{B}}=0 \\
& \vec{\nabla} \times \overrightarrow{\mathcal{B}}-\overrightarrow{\mathcal{E}}^{\prime}=4 \pi e \int d^{3} p \vec{v}\left[f_{+}(\vec{x}, \vec{p}, \eta)-f_{-}(\vec{x}, \vec{p}, \eta)\right] .
\end{aligned}
$$

In order to illustrate the physical relevance of this description, Eqs. (5.38) will be used later in this Section to study the fate of charge and current density fluctuations.

\subsection{Physical scales in the evolution of magnetic fields}

When MHD is discussed in curved spce-times, the evolution of the geometry reflects in the time evolution of the physical scales of the problem.

\subsubsection{Before matter-radiation equality}

From Eq. (5.32), the comoving momentum corresponding to the magnetic diffusivity scale is given by the relation

$$
\frac{k^{2}}{4 \pi \sigma} \sim \frac{1}{\eta} \sim \mathcal{H}=a H
$$

In the case of a relativistic plasma at typical temperatures $T \gg 1 \mathrm{MeV}$, the flat-space conductivity (see Eq. (5.28)) is given by

$$
\sigma_{c} \simeq \frac{\alpha_{\mathrm{em}}}{T \bar{\sigma}}
$$

where $\bar{\sigma} \sim \alpha_{\mathrm{em}}^{2} / T^{2}$ is the cross section ${ }^{19}$. Then, it can be shown [135] that

$$
\sigma_{c}=\sigma_{0} \frac{T}{\alpha_{\mathrm{em}}}
$$

where $\sigma_{0}$ is a slowly increasing function of the temperature. In 135 it has been convincingly argued that $\sigma_{0} \sim 0.06$ at $T \sim 100 \mathrm{MeV}$ while it becomes 0.6 at $T \sim 100 \mathrm{GeV}$ (for a recent analysis of conductivity in high temperature QED see [136]). Recalling Eq. (5.28) the physical diffusivity scale $\omega_{\sigma}=k_{\sigma} / a(\eta)$ will be, in units of the Hubble parameter,

$$
\frac{\omega_{\sigma}}{H} \simeq \sqrt{\frac{4 \pi \sigma_{0}}{\alpha_{\mathrm{em}}}}\left(\frac{T}{H}\right)^{1 / 2}=72.2 \times \sigma_{0}^{1 / 2} g_{*}^{-1 / 4}\left(\frac{T}{M_{\mathrm{P}}}\right)^{-1 / 2} .
$$

\footnotetext{
${ }^{19}$ The cross section is denoted with $\bar{\sigma}$ while the conductivity is $\sigma$.
} 
Discounting for the effect due to the possible variations of the effective relativistic degrees of freedom, the (physical) magnetic diffusivity length scale is much smaller than the Hubble radius at the corresponding epoch. For instance, if $T \sim 10 \mathrm{MeV}$, then $L_{\sigma} \sim \omega_{\sigma}^{-1} \sim 10^{-10} H^{-1}$. This means that there are ten decades length-scales where the magnetic field spectrum will not experience diffusion due to the finite value of the conductivity.

The estimate of the magnetic diffusivity scale will now be repeated in a different range of temperatures, namely for $T<0.2 m_{\mathrm{e}}$ when weak interactions have fallen out of thermal equilibrium. In this case the relevant degrees of freedom are the three neutrino species and the photon, i.e. $g_{*}=3.36$. In this case the conductivity will be given by

$$
\sigma \simeq \frac{\alpha_{\mathrm{em}}}{m_{\mathrm{e}} v_{\mathrm{th}} \bar{\sigma}}
$$

which leads to

$$
\sigma_{\mathrm{c}}=\frac{1}{\alpha_{\mathrm{em}}}\left(\frac{T}{m_{\mathrm{e}}}\right)^{1 / 2} T
$$

if $v_{\mathrm{th}} \sim\left(T / m_{\mathrm{e}}\right)^{1 / 2}$ and $\bar{\sigma} \sim \alpha_{\mathrm{em}}^{2} / T^{2}$. Eq. (5.44) leads to the known $T^{3 / 2}$ dependence of the conductivity from the temperature, as expected from a non-relativistic plasma. The physical magnetic diffusivity momentum is, in this case

$$
\frac{\omega_{\sigma}}{H} \simeq \sqrt{\frac{4 \pi}{\alpha}}\left(\frac{T}{m_{\mathrm{e}}}\right)^{1 / 4} \sqrt{\frac{T}{H}} \sim \frac{11}{\alpha_{\mathrm{em}}} g_{*}^{-1 / 4}\left(\frac{T}{m_{\mathrm{e}}}\right)^{1 / 4}\left(\frac{M_{\mathrm{P}}}{T}\right)^{1 / 2} .
$$

Eqs. (5.42) and (5.45) refer to two different ranges of temperatures. The magnetic diffusivity scale in units of the Hubble parameter obtained in Eq. (5.45) is smaller than the one given by in Eq. (5.42). Hence, by lowering the temperature across the weak equilibration temperature makes the conductivity length scale larger in units of the Hubble radius : inertial range ${ }^{20}$ of length scales (i.e. the region where conductivity effects are negligible) is larger above the weak equilibration temperature. Summarizing

$$
\begin{aligned}
L_{\sigma}(T) & =\frac{\sigma_{0}^{-1 / 2} g_{*}^{1 / 4}}{72.2}\left(\frac{T}{M_{\mathrm{P}}}\right)^{1 / 2} L_{H}(T), \quad T>\mathrm{MeV} \\
L_{\sigma}(T) & =\frac{\sigma_{0}^{-1 / 2} g_{*}^{1 / 4}}{72.2}\left(\frac{T}{M_{\mathrm{P}}}\right)^{1 / 2}\left(\frac{T}{m_{\mathrm{e}}}\right)^{-1 / 4} L_{H}(T), \quad T<\mathrm{MeV},(5.46)
\end{aligned}
$$

\footnotetext{
${ }^{20}$ In more general terms the inertial range is the interval of scales (either in momentum or in real space) where the dynamics is independent on the scales of dissipation so that, in this range, the diffusivities can be taken to zero.
} 
where $L_{H}(T)=H^{-1}(T)$.

In the evolution of the plasma it is sometimes relevant to estimate the thermal diffusivity scale setting a bound on the coherence of the velocity field and not of the magnetic field. There are however phenomena, like the propagation of MHD waves, where the excitations of a background magnetic field are coupled to the excitations of the velocity field. Generally speaking the typical correlation scale of the velocity field is always smaller, in various physical situations, than the typical correlation scale of the magnetic fields. The range of physical momenta affected by thermal diffusivity is bounded from above by the thermal diffusivity scale which can be obtained from the following relations

$$
k^{2} \nu \simeq \frac{k^{2}}{5} \ell_{\gamma}(\eta) \simeq \frac{1}{\eta}=\mathcal{H}=a H,
$$

were $\ell_{\gamma}$ is the photon mean free path, i.e.

$$
\ell_{\gamma} \simeq \frac{1}{n_{\mathrm{e}} \bar{\sigma}}
$$

The mean free path measures the efficiency of a given process to transfer momentum. From Eqs. (5.29) and (5.47) the typical thermal diffusivity momentum is:

$$
\frac{\omega_{\mathrm{diff}}}{H} \simeq \sqrt{\frac{5}{H(T) \ell_{\gamma}(T)}} .
$$

where $\omega_{\text {diff }}=k_{\text {diff }} / a$ is the physical momentum as opposed to the comoving momentum $k_{\text {diff. }}$ The scale defined in Eq. (5.49) is sometimes called Silk scale. Notice that the mean free path given in Eq. (5.48) increases as $a^{3}(\eta)$. Since the physical scale of an inhomogeneous region increases as $a(\eta)$, the photon mean free path can become larger than the physical size of an inhomogeneous region. This is the so-called free streaming regime.

If $T<0.2 m_{\mathrm{e}}$, the photon mean free path is given by Eq. (5.48) where $\bar{\sigma}_{\mathrm{T}}=1.7 \times 10^{3} \mathrm{GeV}^{-2} \simeq \alpha_{\mathrm{em}} m_{\mathrm{e}}^{-2}$ is the Thompson cross section and

$$
n_{\mathrm{e}} \simeq 6.6 \times 10^{-9}\left(\Omega_{\mathrm{b}} h_{0}^{2}\right) x_{\mathrm{e}} T^{3}
$$

is the electron number density depending both on the critical fractions of baryons, $\Omega_{\mathrm{b}}$ and on the ionization fraction $x_{\mathrm{e}}$. The ionization fraction is the ratio between the number density of protons and the total number density 
of baryons. The value of $x_{\mathrm{e}}$ drops from 1 to $10^{-5}$ in a short time around recombination. Eq. (5.50) is simply derived by noticing that, after electron positron annihilation, $n_{\mathrm{e}} \simeq \bar{\eta} n_{\gamma}$ where $\bar{\eta}=2.74 \times 10^{-8} \Omega_{\mathrm{b}} h_{0}^{2}$ is the baryonto-photon ratio, the free parameter of big-bang nucleosynthesis calculations [138, 139, 140, 141]. Thus

$$
\ell_{\gamma}(T) \simeq 8.8 \times 10^{12}\left(\frac{x_{\mathrm{e}}}{0.5}\right)^{-1}\left(\frac{\Omega_{\mathrm{b}} h_{0}^{2}}{0.02}\right)^{-1}\left(\frac{\mathrm{MeV}}{T}\right)^{2} T^{-1} .
$$

For temperatures larger than the $\mathrm{MeV}$ the photon mean free path can be estimated by recalling that, above the $\mathrm{MeV}$, the Thompson cross section is replaced by the Klein-Nishina cross section (i.e. $\bar{\sigma} \sim T^{-1} \mathrm{GeV}^{-1}$ ). Thus, from Eq. (5.48), the photon mean free path is

$$
\ell_{\gamma}(T) \simeq 0.1\left(\frac{\mathrm{GeV}}{T}\right)^{2} \mathrm{GeV}^{-1}
$$

As before, it is useful to refer $\omega_{\text {diff }}$ to the Hubble rate and to compute the typical diffusion distances for $T>1 \mathrm{MeV}$

$$
\frac{L_{\text {diff }}^{(\gamma)}(T)}{L_{H}(T)} \simeq 1.1 \times 10^{-10}\left(\frac{g_{*}}{10.75}\right)^{1 / 4}
$$

and for $T<1 \mathrm{MeV}$

$$
\frac{L_{\text {diff }}^{(\gamma)}(T)}{L_{H}(T)} \simeq 1.03 \times 10^{-5}\left(\frac{\Omega_{\mathrm{b}} h_{0}^{2}}{0.02}\right)^{-1 / 2}\left(\frac{x_{\mathrm{e}}}{0.5}\right)^{-1 / 2}\left(\frac{g_{*}}{10.75}\right)^{1 / 4} \sqrt{\frac{\mathrm{MeV}}{T}} .
$$

At temperatures $\mathcal{O}(\mathrm{MeV})$, corresponding roughly to $e^{+} e^{-}$annihilation, Eqs. (5.53) and (5.54) differ by a factor $10^{5}$. This drop of five orders of magnitude of the thermal diffusivity scale reflects the drop (by ten orders of magnitude) of the photon mean free path as it can be appreciated by comparing Eqs. (5.51) and (5.52) at $T \simeq \mathcal{O}(\mathrm{MeV})$.

For temperatures smaller than the electroweak temperature (i.e. $T \sim 100$ $\mathrm{GeV}$ ) down to the temperature of neutrino decoupling, neutrinos are the species with the largest mean free path and, therefore, the most efficient momentum transporters. Neglecting possible variations of the number of relativistic species the neutrino mean free path will be simply given by $\ell_{\nu}=$ $\mathrm{G}_{\mathrm{F}}^{-2} T^{-2}\left(n_{\text {lep }}+n_{\text {qua }}\right)^{-1}$ where $n_{\text {lep }}$ and $n_{\text {qua }}$ are the leptons and quark number densities. Using this estimate the diffusion scale will be

$$
L_{\text {diff }}^{(\nu)} \simeq 10^{-5}\left(\frac{\mathrm{MeV}}{T}\right)^{3 / 2} L_{H}
$$


which should be compared with Eq. (5.53). Clearly $L_{\text {diff }}^{(\nu)}>L_{\text {diff }}^{(\gamma)}$.

It seems then difficult to get observable effects from inhomogeneities induced by MHD excitations. In [137 the damping rates of Alfvén and magnetosonic waves have been computed and it has been shown that MHD modes suffer significant damping (see, however, [131, 132]). To avoid confusion, the results reported in Ref. 137 apply to the damping of MHD waves and not to the damping of magnetic fields themselves as also correctly pointed out in 142 .

\subsubsection{After matter-radiation equality}

In standard big bang cosmology there are three rather close (but rather different) epochs,

- the matter-radiation equality taking place at $T_{\text {eq }} \simeq 5.5\left(\Omega_{0} h_{0}^{2}\right) \mathrm{eV}$, i.e. $t_{\mathrm{eq}} \simeq 4 \times 10^{10}\left(\Omega_{0} h_{0}^{2}\right)^{-2} \mathrm{sec} ;$

- the decoupling of radiation from matter occurring at $T_{\mathrm{dec}} \simeq 0.26 \mathrm{eV}$ i.e. $t_{\mathrm{dec}} \simeq 5.6 \times 10^{12}\left(\Omega_{0} h_{0}^{2}\right)^{-1 / 2} \mathrm{sec}$

- the recombination occurring, for a typical value of the critical baryon fraction, at $T_{\text {rec }} \simeq 0.3 \mathrm{eV}$, i.e. $t_{\mathrm{rec}} \simeq 4.3 \times 10^{12}\left(\Omega_{0} h_{0}^{2}\right)^{-1 / 2} \mathrm{sec}$.

After plasma recombines, the baryons are no longer prevented from moving by viscosity and radiation pressure. Thus, the only term distinguishing the evolution of the baryons from the evolution of a cold dark matter fluid is the presence of the Lorentz force term in the Navier-Stokes equation. In this situation, it is interesting to investigate the interplay between the forced MHD regime and the generation of gravitational inhomogeneities. It was actually argued long ago by Wasserman [143] and recently revisited by Coles [144] that large-scale magnetic fields may have an important impact on structure formation. Assuming that, for illustrative purposes, the matter fluid consists only of baryons and cold dark matter, for $t>t_{\text {rec }}$ the evolution of the system is described by the following set of non relativistic equations

$$
\begin{aligned}
& \frac{\partial \vec{v}_{\mathrm{b}}}{\partial \eta}+\mathcal{H} \vec{v}_{\mathrm{b}}=-\vec{\nabla} \Phi+\frac{\vec{\nabla} \times \overrightarrow{\mathcal{B}} \times \overrightarrow{\mathcal{B}}}{4 \pi a^{4}(t) \rho_{\mathrm{b}}}, \\
& \frac{\partial \vec{v}_{\mathrm{c}}}{\partial \eta}+\mathcal{H} \vec{v}_{\mathrm{c}}=-\vec{\nabla} \Phi, \\
& \nabla^{2} \Phi=\frac{a^{2}}{2 M_{\mathrm{P}}^{2}}\left[\rho_{\mathrm{b}} \delta_{\mathrm{b}}+\rho_{\mathrm{c}} \delta_{\mathrm{c}}\right],
\end{aligned}
$$


where $\Phi$ is the Newtonian potential. Eqs. (15.56) come from Eq. (5.31) for $\gamma=0$ in the case of homogeneous pressure density. Eq. (5.57) can be obtained, in the same limit, from Eq. Eq. (5.31) by setting to zero the Lorentz force.

From Eq. (5.30), by linearizing the continuity equations for the two species, the relation between the divergence of the velocity field and the density contrast can be obtained

$$
\delta_{\mathrm{b}}^{\prime}=-\vec{\nabla} \cdot \vec{v}_{\mathrm{b}}, \quad \delta_{\mathrm{c}}^{\prime}=-\vec{\nabla} \cdot \vec{v}_{\mathrm{c}},
$$

where $\delta_{\mathrm{b}}=\delta \rho_{\mathrm{b}} / \rho_{\mathrm{b}}$ and $\delta_{\mathrm{c}}=\delta \rho_{\mathrm{c}} / \rho_{\mathrm{c}}$ Thus, taking the divergence of Eqs. (5.56) and (5.57) and using, in the obtained equations, Eqs. (5.58) and (5.59) the following system can be obtained

$$
\begin{aligned}
\delta_{\mathrm{b}}^{\prime \prime}+\mathcal{H} \delta_{\mathrm{b}}-\frac{a^{2}}{2 M_{\mathrm{P}}^{2}} \rho_{\mathrm{m}} \delta & =\frac{\vec{\nabla} \cdot[(\vec{\nabla} \times \overrightarrow{\mathcal{B}}) \times \overrightarrow{\mathcal{B}}]}{4 \pi \rho_{\mathrm{b}} a^{4}(t)}, \\
\delta_{\mathrm{c}}^{\prime \prime}+\mathcal{H} \delta_{\mathrm{c}}-\frac{a^{2}}{2 M_{\mathrm{P}}^{2}} \rho_{\mathrm{m}} \delta & =0,
\end{aligned}
$$

where $\delta=\Omega_{\mathrm{b}} \delta_{\mathrm{b}}+\Omega_{\mathrm{c}} \delta_{\mathrm{c}}$ and $\rho_{\mathrm{m}}=\rho_{\mathrm{b}}+\rho_{\mathrm{c}}$ is the total matter density. Multiplying Eq. (5.60) by $\Omega_{\mathrm{b}}$ and Eq. (5.61) by $\Omega_{\mathrm{c}}$, the sum of the two resulting equations can be written as

$$
\delta^{\prime \prime}+\mathcal{H} \delta-\frac{a^{2}}{2 M_{\mathrm{P}}^{2}} \rho_{\mathrm{m}} \delta=\Omega_{\mathrm{b}} \frac{\vec{\nabla} \cdot[(\vec{\nabla} \times \overrightarrow{\mathcal{B}}) \times \overrightarrow{\mathcal{B}}]}{4 \pi \rho_{\mathrm{b}} a^{4}(t)},
$$

where it has been used that the background geometry is spatially flat. Going to cosmic time, Eq. (5.62) reads

$$
\ddot{\delta}+\frac{2}{3 t} \dot{\delta}-\frac{2}{3 t^{2}} \delta=\frac{\vec{\nabla} \cdot[(\vec{\nabla} \times \overrightarrow{\mathcal{B}}) \times \overrightarrow{\mathcal{B}}]}{4 \pi \rho_{\mathrm{b}} a^{6}(t)} .
$$

The homogeneous term of Eq. (5.63) can be easily solved. The source term strongly depends upon the magnetic field configuration 88 . However, as far as time evolution is concerned, $\overrightarrow{\mathcal{B}}$ is constant in the inertial range of scales, i.e. $L>L_{\sigma}$. Hence, $\overrightarrow{\mathcal{B}}=\overrightarrow{\mathcal{B}_{0}}$ and $\vec{B}(t)=\overrightarrow{\mathcal{B}}_{0} / a^{2}(t)$. Assuming a stochastic magnetic field the solution of equation (5.63) can be obtained integrating from $t_{\text {rec }}$ and recalling that $\rho_{\mathrm{b}}(t) \sim \rho_{\mathrm{b}}\left(t_{\mathrm{rec}}\right) a^{-3}$. The result is

$$
\delta(t)=\Omega_{\mathrm{b}} \frac{t_{\mathrm{rec}}^{2}\left|\vec{B}_{\mathrm{rec}}\right|^{2}}{4 \pi \rho_{\mathrm{b}}\left(t_{\mathrm{rec}}\right) a^{2}\left(t_{\mathrm{rec}}\right) \lambda_{\mathrm{B}}^{2}}\left[\frac{9}{10}\left(\frac{t}{t_{\mathrm{rec}}}\right)^{2 / 3}+\frac{3}{5}\left(\frac{t_{\mathrm{rec}}}{t}\right)-\frac{3}{2}\right] .
$$


where $\lambda_{\mathrm{B}}$ is the typical length scale of variation of the magnetic field. The total density contrast grows as $t^{2 / 3}$ with an initial condition set by the Lorentz force, i. e.

$$
\delta\left(t_{\mathrm{rec}}\right) \simeq \Omega_{\mathrm{b}} \frac{\left|\vec{B}_{\mathrm{rec}}\right|^{2} t_{\mathrm{rec}}^{2}}{4 \pi \rho_{\mathrm{b}}\left(t_{\mathrm{rec}}\right) a^{2}\left(t_{\mathrm{rec}}\right) \lambda_{\mathrm{B}}^{2}} .
$$

To seed galaxy directly the effective $\delta\left(t_{\text {rec }}\right)$ should be of the order off $10^{-3}$ for a flat Universe, implying that

$$
\left|\vec{B}_{\text {rec }}\right| \simeq 10^{-3}\left(\frac{\lambda_{\mathrm{B}}}{\mathrm{Mpc}}\right)\left(\Omega_{\mathrm{b}} h^{2}\right)^{1 / 2} \quad \mathrm{G} .
$$

Also the converse is true. Namely one can read Eq. (5.66) from left to right. In this case Eq. (5.66) will define the typical scale affected by the presence of the magnetic field. This scale is sometimes called magnetic Jeans scale 101]: it can be constructed from the Jeans length

$$
\lambda_{\mathrm{J}}=c_{\mathrm{s}}\left(\frac{\pi}{G \rho_{\mathrm{b}}}\right)^{1 / 2}
$$

where $c_{\mathrm{s}}$ is replaced by $c_{\mathrm{a}}$, i.e. the Alfvén velocity.

\subsection{Charge and current density fluctuations}

As a simple application of kinetic techniques, it is useful to understand which magnetic field can be obtained from an initial inhomogeneous distribution of charge and current density fluctuations.

Consider then the system of Eqs. (5.38) under the assumption that, initially, electric and magnetic fields vanish:

$$
\delta f_{ \pm}\left(\vec{x}, \vec{p}, \eta_{0}\right) \neq 0, \quad \overrightarrow{\mathcal{E}}\left(\vec{x}, \eta_{0}\right)=\overrightarrow{\mathcal{B}}\left(\vec{x}, \eta_{0}\right)=0 .
$$

Using Eqs. (5.36) - (5.38) the induced magnetic fields can be obtained.. This problem is discussed, in greater detail, in [134].

Subtracting Eqs. (5.36) and (5.37), and defining

$$
f(\vec{x}, \vec{p}, \eta)=\delta f_{+}(\vec{x}, \vec{p}, \eta)-\delta f_{-}(\vec{x}, \vec{p}, \eta)
$$

the Vlasov-Landau system can be written as

$$
-g_{\vec{k}}(\vec{p})+i(\vec{k} \cdot \vec{v}-\omega) f_{\vec{k} \omega}(\vec{p})+2 e \overrightarrow{\mathcal{E}}_{\vec{k} \omega} \cdot \frac{\partial f_{0}}{\partial \vec{p}}=-\tilde{\nu} f_{\vec{k}, \omega}
$$




$$
\begin{aligned}
& i \vec{k} \cdot \overrightarrow{\mathcal{E}}_{\vec{k} \omega}=4 \pi e \int f_{\vec{k} \omega}(\vec{p}) d^{3} p, \\
& i \vec{k} \cdot \overrightarrow{\mathcal{B}}_{\vec{k} \omega}=0, \\
& \overrightarrow{\mathcal{B}}_{\vec{k} \omega}=\frac{1}{\omega} \vec{k} \times \vec{E}_{\vec{k} \omega}, \\
& i \omega\left(1-\frac{k^{2}}{\omega^{2}}\right) \overrightarrow{\mathcal{E}}_{\vec{k} \omega}+\frac{i}{\omega} \vec{k}\left(\vec{k} \cdot \overrightarrow{\mathcal{E}}_{\vec{k} \omega}\right)=4 \pi e \int d^{3} p \vec{v} f_{\vec{k} \omega}(\vec{p}),
\end{aligned}
$$

where $g_{\vec{k}}(\vec{p})$ is the initial profile of the distribution function and where $\tilde{\nu}$ is the typical collision frequency. The subscripts $\vec{k} \omega$ denote the Fourier transform with respect to space and the Laplace transform with respect to time of the corresponding quantity.

After enforcing the Gauss constraint at the initial time, the electric field can be separated in its polarizations parallel and transverse to the direction of propagation of the fluctuation. The transverse current provides a source for the evolution of transverse electric field fluctuations

$$
i \omega\left(1-\frac{k^{2}}{\omega^{2}}\right) \overrightarrow{\mathcal{E}}_{\vec{k} \omega}^{\perp}=4 \pi e \int d^{3} p f_{\vec{k} \omega}(\vec{p}) \vec{v}_{\perp},
$$

whereas the charge fluctuations provide a source for the evolution of longitudinal electric field fluctuations

$$
i \vec{k} \cdot \overrightarrow{\mathcal{E}}_{\vec{k} \omega}^{\|}=4 \pi e \int d^{3} p f_{\vec{k} \omega}(\vec{p}) .
$$

In Eqs. (5.751) and (5.76) the longitudinal and transverse part of the electric field fluctuations have been defined:

$$
\overrightarrow{\mathcal{E}}_{\vec{k} \omega}^{\perp}=\overrightarrow{\mathcal{E}}_{\vec{k} \omega}^{\perp}-\frac{\vec{k}}{|\vec{k}|^{2}}\left(\vec{k} \cdot \overrightarrow{\mathcal{E}}_{\vec{k} \omega}\right), \quad \overrightarrow{\mathcal{E}}_{\vec{k} \omega}^{\|}=\frac{\vec{k}}{|\vec{k}|^{2}}\left(\overrightarrow{\mathcal{E}}_{\vec{k} \omega} \cdot \vec{k}\right) .
$$

The solution of Eq. (5.70) is given by

$$
f_{\vec{k} \omega}(\vec{p})=\frac{1}{i(\vec{k} \cdot \vec{v}-\omega-i \tilde{\nu})}\left[g_{\vec{k}}(\vec{p})-2 e \vec{v} \cdot \overrightarrow{\mathcal{E}}_{\vec{k} \omega} \frac{\partial f_{0}}{\partial p}\right],
$$

where we used that $\partial f_{0} / \partial \vec{p} \equiv \vec{v} \partial f_{0} / \partial p$. The longitudinal and transverse components of the electric fluctuations can be obtained by inserting Eq. (5.78) into Eqs. (5.75)-(5.76)

$$
\begin{aligned}
\left|\overrightarrow{\mathcal{E}}_{\vec{k} \omega}^{\|}\right| & =\frac{e}{i k \epsilon_{\|}} \int d^{3} p \frac{g_{\vec{k}}(\vec{p})}{i(\vec{k} \cdot \vec{v}-\omega-i \tilde{\nu})}, \\
\overrightarrow{\mathcal{E}}_{\vec{k} \omega}^{\perp} & =\frac{e \omega}{\omega^{2} \epsilon_{\perp}-k^{2}} \int d^{3} p \vec{v}^{\perp} \frac{g_{\vec{k}}(\vec{p})}{(\vec{k} \cdot \vec{v}-\omega-i \tilde{\nu})},
\end{aligned}
$$


where $\epsilon_{\|}$and $\epsilon_{\perp}$ are, respectively, the longitudinal and transverse part of the polarization tensor

$$
\begin{aligned}
& \epsilon_{\|}(k, \omega)=1-\frac{2 e^{2}}{k^{2}} \int d^{3} p \frac{\vec{k} \cdot \vec{v}}{(\overrightarrow{\vec{k}} \cdot \vec{v}-\omega-i \tilde{\nu})} \frac{\partial f_{0}}{\partial p} \\
& \epsilon_{\perp}(k, \omega)=1-\frac{e^{2}}{\omega} \int d^{3} p \frac{\vec{v}_{\perp}}{(\vec{k} \cdot \vec{v}-\omega-i \tilde{\nu})} \frac{\partial f_{0}}{\partial p} .
\end{aligned}
$$

Now, the general expression for the generated magnetic field is

$$
\overrightarrow{\mathcal{B}}_{\vec{k} \omega}=\frac{e}{\omega^{2} \epsilon_{\perp}(k, \omega)-k^{2}} \int d^{3} p[\vec{v} \times \vec{k}] \frac{g_{\vec{k}}(\vec{p})}{(\vec{k} \cdot \vec{v}-\omega-i \tilde{\nu})} .
$$

The space-time evolution of the magnetic fluctuations can be determined by performing the inverse Laplace and Fourier transforms:

$$
\overrightarrow{\mathcal{B}}(\vec{x}, \eta)=\int e^{-i \omega \eta} \frac{e d \omega}{\omega^{2} \epsilon_{\perp}(k, \omega)-k^{2}} \int d^{3} k e^{i \vec{k} \cdot \vec{x}}[\vec{v} \times \vec{k}] \int d^{3} p \frac{g_{\vec{k}}(\vec{p})}{(\vec{v} \cdot \vec{k}-\omega-i \tilde{\nu})} .
$$

In order to perform this integral, the explicit relations for the polarization tensors should be given. They depend on the equilibrium distribution function $f_{0}(p)$, which we take to be the expression given in Eq. $(5.34)^{21}$

Then we have for transverse polarization

$$
\epsilon_{\perp}(k, \omega)=1+\frac{e^{2} n_{q}}{2 \omega k T}\left\{\left[1-\frac{(\omega+i \tilde{\nu})^{2}}{k^{2}}\right] \ln \frac{k-\omega-i \tilde{\nu}}{k+\omega+i \tilde{\nu}}-2 \frac{\omega+i \tilde{\nu}}{k}\right\},
$$

and for the longitudinal polarization:

$$
\epsilon_{\|}(k, \omega)=1+\frac{e^{2} n_{q}}{k^{2} T}\left\{2+\frac{\omega+i \tilde{\nu}}{k} \ln \frac{k-\omega-i \tilde{\nu}}{k+\omega+i \tilde{\nu}}\right\} .
$$

Consider now the case of very small momenta $k \ll \omega$ and $\omega \ll \tilde{\nu}$, relevant for long-ranged magnetic fields. Then the computation of the integral (5.84) in the large time limit and with the use of explicit form of the transverse polarization tensor in (5.85) gives ${ }^{22}$ :

$$
\overrightarrow{\mathcal{B}}(\vec{x}, \eta) \simeq \frac{T}{4 \pi \alpha_{\mathrm{em}} n_{q}} \exp \left(-k^{2} \eta / \sigma\right) \vec{\nabla} \times \overrightarrow{\mathcal{J}},
$$

\footnotetext{
${ }^{21}$ Notice that most of our considerations can be easily extended to the case of a BoseEinstein or Fermi-Dirac distribution. What is important, in our context, is the analytical structure of the polarization tensors and this is the same for different distributions [125].

${ }^{22}$ For small $k$, the equation $\omega^{2} \epsilon_{\perp}(k, \omega)-k^{2}=0$ defining the poles of the inverse Laplace transform implies $\omega \sim i k^{2} / \sigma$.
} 
where $\sigma$ is the plasma conductivity in the relaxation time approximation,

$$
\sigma=\frac{4 \pi e^{2} n_{q}}{\tilde{\nu} T}
$$

and initial electric current is given by

$$
\overrightarrow{\mathcal{J}}(\vec{x})=\int d^{3} p \vec{v} g_{\vec{k}}(\vec{p})
$$

The obtained results assumed that the linearization of the Vlasov equation is consistent with the physical assumptions of our problem. This is indeed the case. In order to safely linearize the Vlasov equation we have to make sure that the perturbed distribution function of the charge fluctuations is always smaller than the first order of the perturbative expansion (given by the distribution of Eq. 5.34). In other words we have to make sure that

$$
\left|\delta f_{+}(\vec{x}, \vec{p}, \eta)\right| \ll f_{0}(\vec{p}), \quad\left|\delta f_{-}(\vec{x}, \vec{p}, \eta)\right| \ll f_{0}(\vec{p}) .
$$

These conditions imply that

$$
\frac{e \overrightarrow{\mathcal{E}}_{\vec{k} \omega}}{|\vec{k} \cdot \vec{v}-\omega-i \tilde{\nu}|} \cdot \frac{\partial f_{0}(\vec{p})}{\partial \vec{p}} \ll f_{0}(\vec{p}) .
$$

If the relativistic plasma frequency is defined,

$$
\omega_{p}^{2}=\frac{4 \pi e^{2} n_{q}}{3 T}
$$

the condition expressed by Eq. (5.91) can be restated, for modes $k \leq \omega_{p}$, as $\left|\overrightarrow{\mathcal{E}}_{\vec{k}, \omega}\right|^{2}<n_{q} T$ (where we essentially took the square modulus of Eq. (5.91)). This last inequality expresses the fact that the energy density associated with the gauge field fluctuations should always be smaller than the critical energy density stored in radiation. The linear treatment of the Vlasov equation is certainly accurate provided the typical modes of the the field are smaller than the plasma frequency and provided the energy density in electric and magnetic fields is smaller than $T^{4}$, i.e. the energy density stored in the radiation background. 


\subsection{Inverse cascades}

A relevant topic in the evolution of large scale magnetic fields concerns the concepts of direct and inverse cascades. A direct cascade is a process, occurring in a plasma, where energy is transferred from large to small length scales. An inverse cascade is a process where the energy transfer goes from small scales to large length scales. One can also generalize the the concept of energy cascade to the cascade of any conserved quantity in the plasma, like, for instance, the helicity. Thus, in general terms, the transfer process of a conserved quantity is a cascade.

The concept of cascade (either direct or inverse) is related with the concept of turbulence, i.e. the class of phenomena taking place in fluids and plasmas at high Reynolds numbers, as anticipated in Section 4. Non-magnetized fluids become turbulent at high kinetic Reynolds numbers. Similarly, magnetized fluids should become turbulent for sufficiently large magnetic Reynolds numbers. The experimental evidence of this occurrence is still poor. One of the reasons is that it is very difficult to reach, with terrestrial plasmas, the physical situation where the magnetic and the kinetic Reynolds numbers are both large but, in such a way that their ratio is also large i.e.

$$
\mathrm{R}_{\mathrm{m}} \gg 1, \quad \mathrm{R} \gg 1, \quad \mathrm{Pr}=\frac{\mathrm{R}_{\mathrm{m}}}{\mathrm{R}} \gg 1 .
$$

The physical regime expressed through Eqs. (5.93) rather common in the early Universe. Thus, MHD turbulence is probably one of the key aspects of magnetized plasma dynamics at very high temperatures and densities. Consider, for instance, the plasma at the electroweak epoch when the temperature was of the order of $100 \mathrm{GeV}$. One can compute the Reynolds numbers and the Prandtl number from their definitions given in Eqs. (4.40)-(4.42). In particular,

$$
\mathrm{R}_{\mathrm{m}} \sim 10^{17}, \quad \mathrm{R}=10^{11}, \quad \mathrm{Pr} \simeq 10^{6},
$$

which can be obtained from Eqs. (4.40)-4.42) using as fiducial parameters $v \simeq 0.1, \sigma T / \alpha, \nu \simeq(\alpha T)^{-1}$ and $L \simeq 0.01 H_{\mathrm{ew}}^{-1} \simeq 0.03 \mathrm{~cm}$ for $T \simeq 100 \mathrm{GeV}$.

At high Reynolds and Prandtl numbers the evolution of the large-scale magnetic fields is not obvious since the inertial regime is large. There are then two scales separated by a huge gap: the scale of the horizon $(3 \mathrm{~cm}$ at the electroweak epoch) and the diffusivity scale (about $10^{-8} \mathrm{~cm}$ ). One would like to know, given an initial spectrum of the magnetic fields, what is the evolution of the spectrum, i.e. in what modes the energy has been 
transferred. The problem is that it is difficult to simulate systems with huge hierarchies of scales.

Suppose, for instance, that large scale magnetic fields are generated at some time $t_{0}$ and with a given spectral dependence. The energy spectrum of magnetic fields may receive, for instance, the dominant contribution for large comoving momentum $k$ (close to the cut-off provided by the magnetic diffusivity scale). The "initial" spectrum at the time $t_{0}$ is sometimes called injection spectrum. The problem will then be to know the how the spectrum is modified at a later time $t$. If inverse cascade occurs, the magnetic energy density present in the ultra-violet modes may be transferred to the infra-red modes. In spite of the fact that inverse cascades are a powerful dynamical principle, it is debatable under which conditions they may arise.

The possible occurrence of inverse cascades in a magnetized Universe has been put forward, in a series of papers, by Brandenburg, Enqvist and Olesen [145, 146, 147, 148, (see also [149] for an excellent review covering also in a concise way different mechanisms for the generation of large-scale magnetic fields).

MHD simulations in $2+1$ dimensions [145, 146] seem to support the idea of an inverse cascade in a radiation dominated Universe. Two-dimensional MHD simulations are very interesting but also peculiar. In $2+1$ dimensions, the magnetic helicity does not exist while the magnetic flux is well defined and conserved in the ideal MHD limit. It is therefore impossible to implement 2+1-dimensional MHD simulations where the magnetic flux and the magnetic helicity are constrained to be constant in the ideal limit. This technical problem is related to a deeper physical difference between magnetized plasmas with and without helicity. Consider then, for the moment, the situation where the helicity is strictly zero. It is indeed possible to argue that, in this case, $2+1$-dimensional MHD simulations can capture some of the relevant features of $3+1$-dimensional MHD evolution.

In 147, 148] the scaling properties of MHD equations have been investigated in the force-free regime (i.e. in the approximation of negligible Lorentz force). In the absence of helical components at the onset of the MHD evolution a rather simple argument has been proposed by Olesen [147] in order to decide under which conditions inverse cascade may arise. Consider the magnetic diffusivity and Navier-Stokes equations in the limit where $\overrightarrow{\mathcal{J}} \times \overrightarrow{\mathcal{B}} \simeq 0$. Then, the evolution equations are invariant under the following similarity 
transformations:

$$
\begin{aligned}
& \vec{x} \rightarrow L \vec{x}, \quad \eta \rightarrow L^{1-\epsilon} \eta, \quad \vec{v} \rightarrow L^{\epsilon} \vec{v} \\
& \overrightarrow{\mathcal{B}} \rightarrow L^{\epsilon} \overrightarrow{\mathcal{B}}, \quad \nu \rightarrow L^{1+\epsilon} \nu, \quad \sigma \rightarrow L^{-1-\epsilon} \sigma .
\end{aligned}
$$

For simplicity it is also useful to concentrate the attention on the inertial range of momenta where the dynamics is independent on the scale of dissipation.

In order to understand correctly the argument under discussion the volume average of the magnetic energy density has to be introduced (see [150] for a lucid discussion on the various possible averages of large-scale magnetic fields and see [151] for a complementary point of view). A generic rotationally and parity invariant two-point function for the magnetic inhomogeneities can be written as

$$
\mathcal{G}(\vec{r}, \eta)=\langle\overrightarrow{\mathcal{B}}(\vec{x}, \eta) \overrightarrow{\mathcal{B}}(\vec{y}, \eta)\rangle, \quad \vec{r}=\vec{x}-\vec{y} .
$$

Defining now

$$
\mathcal{G}(\vec{r}, \eta)=\int d^{3} k \mathcal{G}(k, \eta) e^{-i \vec{k} \cdot \vec{r}}
$$

From Eqs. (5.96) - (15.97) the following relation can be obtained

$$
V \int d k \mathcal{Q}_{k}(\eta)=\int d^{3} x d^{3} y\langle\overrightarrow{\mathcal{B}}(\vec{x}, \eta) \overrightarrow{\mathcal{B}}(\vec{y}, \eta)\rangle
$$

where $\mathcal{Q}_{k}(\eta)$ is related to $\mathcal{G}(k, \eta)$ and $V$ is the averaging volume.

The idea is now to study the scaling properties of $\mathcal{Q}_{k}(\eta)$ under the similarity transformations given in Eq. (5.95). Following [147], it is possible to show that

$$
\begin{aligned}
a^{4}(\eta) \mathcal{Q}_{k}(\eta) & =k^{\alpha} F(x) \\
x & =k^{(3+\alpha) / 2} \eta .
\end{aligned}
$$

with $\alpha=-1-2 \epsilon$. In Eq. (5.99) $\mathcal{Q}_{k}(\eta)$ is related to the magnetic energy density and $F(x)$ is a function of the single argument $x=\left(k^{(3+\alpha) / 2} \eta\right)$. If, initially, the spectrum of the magnetic energy density is $k^{\alpha}$, then at later time the evolution will be dictated by $F(x)$. Since $a^{4}(\eta) \mathcal{Q}_{k}(\eta)$ is approximately constant, because of flux conservation, then the comoving momentum will evolve, approximately, as

$$
k \simeq \eta^{-2 /(3+\alpha)} \simeq t^{-1 /(3+\alpha)},
$$


while the physical momentum will scale like

$$
\omega \simeq \eta^{-(5+\alpha) /(\alpha+3)} \sim t^{-(5+\alpha) /(2 \alpha+6)} .
$$

The conclusion of this argument is the following:

- if $\alpha<-3$ the cascade will be direct (or forward);

- if $\alpha>-3$ the comoving momentum moves toward the infra-red and the cascade will be inverse;

- if $\alpha=-3$, then, $\epsilon=1$ and, from Eqs. (5.95)-(5.99), the momentum and time dependence will be uncorrelated.

The Olesen scaling argument has interesting implications:

- if the initial spectrum of the magnetic energy density is concentrated at high momenta then, an inverse cascade is likely;

- from Eqs. (5.100) and (5.101) the typical correlation scale of the magnetic field may, in principle, evolve faster than in the case where the inverse cascade is not present.

In a FRW Universe the correlation scale evolves as $\ell(\eta)=\ell\left(\eta_{0}\right) a(\eta)$ from a given initial time $\eta_{0}$ where magnetic energy density is injected with spectral dependence $k^{\alpha}$. If $\alpha>-3$, Eqs. (5.100)-(5.101) imply that

$$
\ell(\eta)=\ell\left(\eta_{0}\right) a(\eta)\left(\frac{\eta}{\eta_{0}}\right)^{\frac{2}{\alpha+3}} \simeq \ell\left(\eta_{0}\right)\left(\frac{\eta}{\eta_{0}}\right)^{\frac{\alpha+5}{\alpha+3}}
$$

where the second equality follows in a radiation dominated phase of expansion. In flat space, the analog of Eqs. (5.100)-(5.101) would imply that $\ell(t) \sim \ell\left(t_{0}\right)\left(t / t_{0}\right)^{2 /(\alpha+3)}$. Finally, in the parametrization of Olesen [147] the case of a Gaussian random field corresponds to $\alpha=2$, i.e. $\epsilon=-3 / 2$. In this case the correlation scale will evolve as

$$
\ell(\eta)=\ell\left(\eta_{0}\right) a(\eta)\left(\frac{\eta}{\eta_{0}}\right)^{2 / 5} .
$$

In the context of hydrodynamics the scaling law expressed through Eq.(5.103) is also well known [153]. It is appropriate, at this point, to recall that Hogan 
[152 was the first one to discuss the evolution of the correlation scale produced by a Gaussian random injection spectrum (i.e. $\alpha=2$ ). Hogan was also the first one to think of the first order phase transitions as a possible source of large scale magnetic fields [152].

An argument similar to the one discussed in the case of MHD can be discussed in the case of ordinary hydrodynamics. In this case, the analog of $\mathcal{Q}_{k}(\eta)$ will be related to the kinetic energy density whose spectrum, in the case of fully developed turbulence goes as $k^{-5 / 3}$, i.e. the usual Kolmogorov spectrum.

The evolution of the correlation scale derived in Eq. (5.103) can be obtained through another class of arguments usually employed in order to discuss inverse cascades in MHD: the mechanism of selective decay [87] which can be triggered in the context of magnetic and hydrodynamical turbulence. The basic idea is that modes with large wave-numbers decay faster than modes whose wavenumber is comparatively small. Son [142] applied these considerations to the evolution of large-scale magnetic fields at high temperatures and his results (when the magnetic field configuration has, initially, vanishing helicity) are in agreement with the ones obtained in [147, 148]. In [154] the topics discussed in [147, 142] have been revisited and in [155] the possible occurrence of inverse cascades has been criticized using renormalization group techniques.

The main assumption of the arguments presented so far is that the evolution of the plasma occurs in an inertial regime. As discussed above in this Section, at high temperatures the inertial regime is rather wide both from the magnetic end thermal points of view. However, in the case where the magnetic and thermal diffusivity scales are finite the arguments relying on the inertial regime cannot be, strictly speaking, applied. In this situation numerical simulations should be used in order to understand if the diffusive regime either precedes or follows the inverse cascade.

If the magnetic and thermal diffusivity scales are finite, two conceptually different possibilities can be envisaged. One possibility is that the inverse cascade terminates with a diffusive regime. The other possibility is that the onset of diffusion prevents the occurrence of the inverse cascade. This dilemma can be solved by numerical simulations along the lines of the ones already mentioned [145, 146]. The numerical simulations presented in [145, 146] are in $(2+1)$ dimensions. The results support the occurrence of inverse cascade in the presence of large kinetic viscosity. The moment at which the cascade stops depends on the specific value of the viscosity coefficient. 


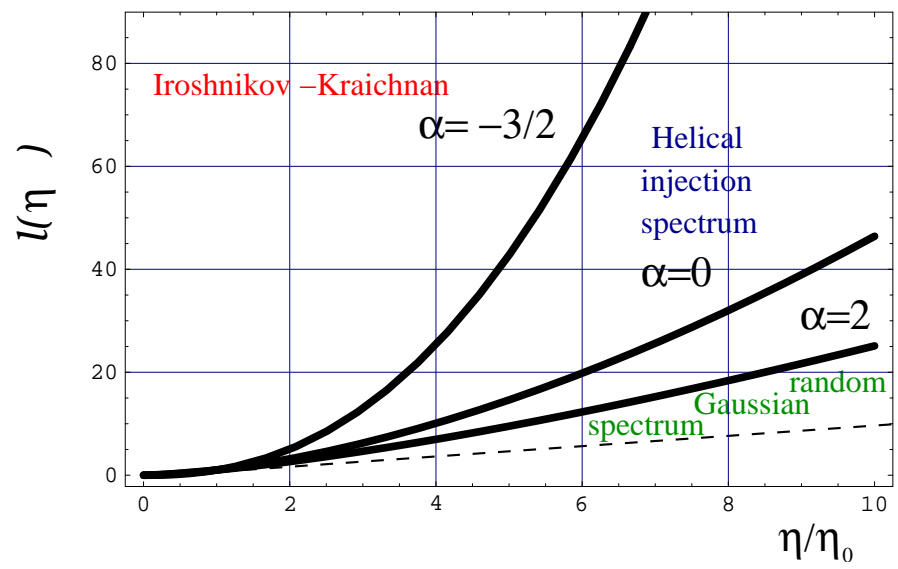

Figure 5: The time evolution of the correlation scale is illustrated in the case of a FRW Universe dominated by radiation and in units $\ell_{0}=1$. The case $\alpha=2$ corresponds to the behaviour obtained in (5.103). The case $\alpha=2$, corresponding to Eq. (5.104), refers to the situation of inverse cascade with an initial helical spectrum. For comparison the case $\alpha=-3 / 2$ is also reported. This last evolution takes place if the injection spectrum is of Iroshnikov-Kraichnan type.

In 156, 157] $(3+1)$-dimensional MHD simulations have shown evidence of inverse cascade. Another possible limitation is that in the early Universe the magnetic and kinetic Reynolds numbers are both large and also their ratio, the Prandtl number, is very large. This feature seems to be difficult to include in simulations.

The considerations presented so far do not take into account the possibility that the initial magnetic field configuration has non vanishing helicity. If magnetic helicity is present the typical features of MHD turbulence can qualitatively change 87] and it was argued that the occurrence of inverse cascades may be even more likely [158, 159] (see also [160]). The conservation of the magnetic flux is now supplemented, in the magnetic sector of the evolution, by the conservation of the helicity obtained in Eqs. (4.25) and (4.27). The conservation of the helicity is extremely important in order to derive the MHD analog of Kolmogorov spectrum. In fact, the heuristic derivation of the Kolmogorov spectrum is based on the conservation of the kinetic energy density. If the conservation of the magnetic helicity is postulated, the same qualitative argument leading to the Kolmogorov spectrum will lead 
in the case of fully developed MHD turbulence to the so-called IroshnikovKraichnan spectrum [87, 161, 162] whose specific form, is, in terms of the quantity introduced in Eq. (5.99) $\mathcal{Q}_{k}\left(\eta_{0}\right) \sim k^{-3 / 2}$.

Suppose, for simplicity, that the injection spectrum has a non-vanishing helicity and suppose that the helicity has the same sign everywhere. In this case $\overrightarrow{\mathcal{A}} \cdot \overrightarrow{\mathcal{B}} \sim \ell|\overrightarrow{\mathcal{B}}|^{2}$. Since the helicity is conserved the magnetic field will scale according to Eqs. (5.95) but with $\epsilon=-1 / 2$ corresponding to $\alpha=0$. Hence, the correlation scale will evolve, in this case, as

$$
\ell(\eta)=\ell\left(\eta_{0}\right) a(\eta)\left(\frac{\eta}{\eta_{0}}\right)^{\frac{2}{3}} \simeq \ell\left(\eta_{0}\right)\left(\frac{\eta}{\eta_{0}}\right)^{\frac{5}{3}},
$$

i.e. faster than in the case of Eq. (5.103). It is difficult to draw general conclusions without specifying the nature and the features of the mechanism producing the injection configuration of the magnetic field. The results critically summarized in this Section will be important when discussing specific ideas on the origin of large-scale magnetic fields. In Fig. [ 5 the cases of different injection spectra are compared. It is clear that an injection spectrum given by a Gaussian random field leads to a rather inefficient inverse cascade. Since the magnetic helicity vanishes at the onset of the evolution, the rate of energy transfer from small to large scales is rather slow. On the contrary if the injection spectrum is helical, the rate increases significantly. For comparison the situation where the injection spectrum corresponds to the Iroshnikov-Kraichnan case, i.e. the case of fully developed MHD turbulence, is also reported. The dashed line of Fig. 5 illustrates the evolution of the correlation scale dictated by the the Universe expansion.

\subsection{Hypermagnetic fields}

At small temperatures and small densities of the different fermionic charges the $S U_{L}(2) \otimes U_{Y}(1)$ is broken down to the $U_{\mathrm{em}}(1)$ and the long range fields which can survive in the plasma are the ordinary magnetic fields. For sufficiently high temperatures (and for sufficiently high values of the various fermionic charges) the $S U_{L}(2) \otimes U_{Y}(1)$ is restored and non-screened vector modes correspond to hypermagnetic fields. In fact, Abelian electric fields decay within a typical time scale $1 / \sigma$ where $\sigma$ is the conductivity. The longranged non-Abelian magnetic fields (corresponding, for instance, to the color $S U(3)$ or to the weak $S U(2)$ ) cannot exist because at high temperatures 
the non-Abelian interactions induce a "magnetic" mass gap $\sim g^{2} T$ where $g$ is the gauge coupling constant. Also the non-Abelian electric fields decay because of the finite value of the conductivity as it occurs for Abelian electric fields. Therefore, the only long scale field that can exist in the plasma for enough time must be associated with some Abelian U(1) group. This statement, valid to all orders in perturbation theory, has been confirmed non-perturbatively for the electroweak theory by recent lattice studies in [165. Under normal conditions (i.e. small temperatures and small densities of the different fermionic charges) the $\mathrm{SU}(2) \times \mathrm{U}(1)_{Y}$ symmetry is "broken" down to $\mathrm{U}(1)_{E M}$, the massless field corresponding to $\mathrm{U}(1)_{E M}$ is the ordinary photon and the only long-lived field in the plasma is the ordinary magnetic one. At sufficiently high temperatures, $T>T_{c}$, the $\mathrm{SU}(2) \times \mathrm{U}(1)_{Y}$ symmetry is "restored", and non-screened vector modes $Y_{\mu}$ correspond to the $\mathrm{U}(1)_{Y}$ hypercharge group. Hence, if primordial fields existed at $T>T_{c}$, they did correspond to hypercharge rather than to $\mathrm{U}(1)_{E M}$.

At the electroweak epoch the typical size of the Hubble radius is of the order of $3 \mathrm{~cm}$. The typical diffusion scale is of the order of $10^{-9} \mathrm{~cm}$. Therefore, over roughly eight orders of magnitude hypermagnetic fields can be present in the plasma without being dissipated [163].

\subsubsection{Anomalous MHD equations}

The evolution of hypermagnetic fields can be obtained from the anomalous magnetohydrodynamical (AMHD) equations. The AMHD equations generalize the treatment of plasma effects involving hypermagnetic fields to the case of finite fermionic density 164.

There are essential differences between the interactions of magnetic fields and the ones of hypermagnetic fields with matter. The ordinary electromagnetic field has a vector-like coupling to the fermions, while the coupling of the hypercharge fields is chiral. Thus, if hyperelectric $\left(\vec{E}_{Y}\right)$ and hypermagnetic $\left(\vec{H}_{Y}\right)$ fields are present simultaneously, they cause a variation of the fermionic number according to the anomaly equation, $\partial_{\mu} j_{\mu} \sim \frac{g^{\prime 2}}{4 \pi^{2}} \vec{H}_{Y} \cdot \vec{E}_{Y}$ (here $g^{\prime}$ the hypercharge gauge coupling constant). Now, the presence of non-homogeneous hypermagnetic fields in the EW plasma with (hyper)conductivity $\sigma_{c}$ always implies the existence of a related electric field, $\vec{E}_{Y} \sim \frac{1}{\sigma_{c}} \vec{\nabla} \times \vec{H}_{Y}$. Since for a general stochastic magnetic background $\left\langle\left(\vec{H}_{Y} \cdot \vec{\nabla} \times \vec{H}_{Y}\right)^{2}\right\rangle \neq 0$, the nonuniform hypermagnetic field may absorb or release fermions and produce, 
ultimately, baryon and lepton density perturbations because of the anomaly equation.

The behaviour of cold fermionic matter with non-zero anomalous Abelian charges was considered in [166 where it was pointed out that the anomalous fermionic matter is unstable against the creation of Abelian gauge field with non-zero Chern-Simons number, which eats up fermions because of the anomaly. The right electron number density may be converted to the hypercharge field because of a similar effect. Also the opposite effect is possible: hypercharge fields may be converted into fermions in a hot environment.

The electroweak plasma in complete thermal equilibrium at a temperature $T$ can be characterized by $n_{f}$ chemical potentials $\mu_{i}, i=1, \ldots, n_{f}$ corresponding to the exactly conserved global charges

$$
N_{i}=L_{i}-\frac{B}{n_{f}}
$$

( $L_{i}$ is the lepton number of the $i$-th generation, $B$ is the baryon number, and $n_{f}$ is the number of fermionic generations). One should also introduce a chemical potential $\mu_{Y}$ corresponding to weak hypercharge, but its value is fixed from the requirement of the hypercharge neutrality of the plasma, $\langle Y\rangle=0$.

It is interesting to study this plasma slightly out of thermal equilibrium, for instance in the situation where a non-uniform distribution of the hypermagnetic field is present. Because of the anomaly, this field is coupled to the fermionic number densities. In principle, different chemical potentials can be assigned to all the fermionic degrees of freedom of the electroweak theory (45 if $n_{f}=3$ ) and the coupled system of Boltzmann-type equations for these chemical potentials and the hypercharge fields may be written. Since we are interested in the slow processes in the plasma, this is not necessary. If the coupling, corresponding to some slow process, is switched off, then the electroweak theory acquires an extra conserved charge and a further chemical potential should be added to the system given in Eq. (5.105).

An interesting observation (which turns out to be quite important in our context) has been made in [167, 168, 169, where it was noticed that perturbative reactions with right-handed electron chirality flip are out of thermal equilibrium at temperatures higher than some temperature $T_{R}{ }^{23}$ Thus, the

\footnotetext{
${ }^{23}$ This temperature depends on the particle physics model, see also the discussion reported in Section 5. In the MSM $T_{R} \simeq 80 \mathrm{TeV}$ [167, 168, 169].
} 
number of right electrons is perturbatively conserved at temperatures $T>T_{R}$ and the chemical potential $\mu_{R}$ can be introduced for it. On the other hand, this charge is not conserved because of the Abelian anomaly,

$$
\partial_{\mu} j_{R}^{\mu}=-\frac{g^{\prime 2} y_{R}^{2}}{64 \pi^{2}} \mathcal{Y}_{\mu \nu} \tilde{\mathcal{Y}}^{\mu \nu}
$$

and it is therefore coupled to the hypermagnetic field. Here $\mathcal{Y}$ and $\tilde{\mathcal{Y}}$ are, respectively, the $U_{Y}(1)$ hypercharge field strengths and their duals, $g^{\prime}$ is the associated gauge coupling and $y_{R}=-2$ is the hypercharge of the right electron.

Now we are ready to derive the anomalous MHD equations in flat space [163, 164. The effective Lagrangian density describing the dynamics of the gauge fields at finite fermionic density is [170]:

$$
\mathcal{L}_{Y, e_{R}}=-\frac{1}{4} \sqrt{-g} Y_{\alpha \beta} Y^{\alpha \beta}-\sqrt{-g} J_{\alpha} Y^{\alpha}+\mu \epsilon_{i j k} Y^{i j} Y^{k}, \quad \mu=\frac{g^{\prime 2}}{4 \pi^{2}} \mu_{R}
$$

( $g$ is the determinant of the metric defined in (5.1); $Y_{\alpha \beta}=\nabla_{[\alpha} Y_{\beta]} ; \nabla_{\alpha}$ is the covariant derivative with respect to the metric (5.1) [notice that in the metric (5.1) $\left.\nabla_{[\alpha} Y_{\beta]}=\partial_{[\alpha} Y_{\beta]}\right] ; g^{\prime}$ is the Abelian coupling constant). The first and the last terms in Eq. (5.107) are nothing but the curved space generalization of the flat-space effective Lagrangian for the hypercharge fields at finite fermion density [163, 164, $J_{\alpha}$ is the ohmic current. The equations of motion for the hyperelectric and hypermagnetic fields are then

$$
\begin{aligned}
& \frac{\partial \overrightarrow{\mathcal{H}}_{Y}}{\partial \eta}=-\vec{\nabla} \times \overrightarrow{\mathcal{E}}_{Y}, \quad \frac{\partial \overrightarrow{\mathcal{E}}_{Y}}{\partial \eta}+\overrightarrow{\mathcal{J}}_{Y}=\frac{g^{\prime 2}}{\pi^{2}} \mu_{R} a \overrightarrow{\mathcal{H}}_{Y}+\vec{\nabla} \times \overrightarrow{\mathcal{H}}_{Y}, \\
& \vec{\nabla} \cdot \overrightarrow{\mathcal{H}}_{Y}=0, \quad \vec{\nabla} \cdot \overrightarrow{\mathcal{E}}_{Y}=0, \\
& \vec{\nabla} \cdot \overrightarrow{\mathcal{J}}_{Y}=0, \quad \overrightarrow{\mathcal{J}}_{Y}=\sigma\left(\overrightarrow{\mathcal{E}}_{Y}+\vec{v} \times \overrightarrow{\mathcal{H}}_{Y}\right), \quad \sigma=\sigma_{c} a(\eta),
\end{aligned}
$$

with the same notations introduced in the case of the conventional MHD equations.

To Eqs. (5.108), the evolution equation of the right electron chemical potential, accounting for the anomalous and perturbative non-conservation of the right electron number density $\left(n_{R}\right)$, must be added:

$$
\frac{\partial n_{R}}{\partial t}=-\frac{g^{\prime 2}}{4 \pi^{2}} \overrightarrow{\mathcal{E}}_{Y} \cdot \overrightarrow{\mathcal{H}}_{Y}-\Gamma\left(n_{R}-n_{R}^{e q}\right),
$$


where $\Gamma$ is the perturbative chirality-changing rate, $\Gamma=T \frac{T_{R}}{M_{0}}, n_{R}^{e q}$ is the equilibrium value of the right electron number density, and the term proportional to $\vec{E}_{Y} \cdot \vec{H}_{Y}$ is the right electron anomaly contribution.

Finally, the relationship between the right electron number density and the chemical potential must be specified. This relation depends upon the particle content of the theory. In the case of the Minimal Standard Models (MSM) the evolution equation of the chemical potential becomes 164

$$
\frac{1}{a} \frac{\partial\left(\mu_{R} a\right)}{\partial \eta}=-\frac{g^{\prime 2}}{4 \pi^{2}} \frac{783}{88} \frac{1}{a^{3} T^{3}} \overrightarrow{\mathcal{E}}_{Y} \cdot \overrightarrow{\mathcal{H}}_{Y}-\Gamma\left(\mu_{R} a\right)
$$

At finite hyperconductivity (in what we would call, in a MHD context, resistive approximation) we have that from Eq. (5.108) the induced hyperelectric field is not exactly orthogonal to the hypermagnetic one and, moreover, an extra "fermionic" current comes in the game thanks to the fact that we are working at finite chemical potential. Therefore in our context the resistive Ohm law can be written as

$$
\begin{aligned}
& \overrightarrow{\mathcal{E}}_{Y}=\frac{\overrightarrow{\mathcal{J}}_{Y}}{\sigma}-\vec{v} \times \overrightarrow{\mathcal{H}}_{Y} \simeq \\
& \frac{1}{\sigma}\left(\frac{4 \alpha^{\prime}}{\pi} \mu_{R} a \overrightarrow{\mathcal{H}}_{Y}+\vec{\nabla} \times \overrightarrow{\mathcal{H}}_{Y}\right)-\vec{v} \times \overrightarrow{\mathcal{H}}_{Y}, \quad \alpha^{\prime}=\frac{g^{\prime 2}}{4 \pi} .
\end{aligned}
$$

In the bracket appearing in Eq. (5.111) we can identify two different contributions. One is associated with the curl of the magnetic field. We will call this the MHD contribution, since it appears in the same way in ordinary plasmas. The other contribution contains the chemical potential and it is directly proportional to the magnetic field and to the chemical potential. This is a typical finite density effect. In fact the extra Ohmic current simply describes the possibility that the energy sitting in real fermionic degrees of freedom can be transferred to the hypermagnetic field. It may be of some interest to notice the analogy between the first term of Eq. (5.111) and the typical form of the ohmic current discussed in Eq. (4.17) appearing in the context of the dynamo mechanism. In the latter case the presence of a current (proportional to the vorticity through the $\alpha$ dynamo term) was indicating that large length scales magnetic fields could grow by eating up fluid vortices. By inserting $\vec{E}_{Y}$ obtained from the generalized Ohm law (5.111) in the evolution equations (5.108) of the hypercharge fields, we obtain the 
generalized form of the magnetic diffusivity equation:

$$
\frac{\partial \overrightarrow{\mathcal{H}}_{Y}}{\partial \eta}=-\frac{4 a \alpha^{\prime}}{\pi \sigma} \vec{\nabla} \times\left(\mu_{R} \overrightarrow{\mathcal{H}}_{Y}\right)+\vec{\nabla} \times(\vec{v} \times \overrightarrow{\mathcal{H}})+\frac{1}{\sigma} \nabla^{2} \overrightarrow{\mathcal{H}}_{Y}
$$

In order to be consistent with our resistive approach we neglected terms containing time derivatives of the electric field, which are sub-leading provided the conductivity is finite. In our considerations we will also make a further simplification, namely we will assume that the EW plasma is (globally) parity-invariant and that, therefore, no global vorticity is present. Therefore, since the length scale of variation of the bulk velocity field is much shorter than the correlation distance of the hypermagnetic field, the infrared modes of the hypercharge will be practically unaffected by the velocity of the plasma, which will be neglected when the large-scale part of the hypercharge is concerned. This corresponds to the usual MHD treatment of a mirror symmetric plasma (see, e.g. Eq. (4.32)-(4.34), when $\alpha=0$ ).

Eqs. (5.112) and (5.110) form a set of AMHD equations for the hypercharge magnetic field and right electron chemical potential in the expanding Universe.

\subsubsection{Hypermagnetic knots}

The Abelian nature of the hypercharge field does not forbid that the hypermagnetic flux lines should have a trivial topological structure. This situation is similar to what already encountered in the case of conventional electromagnetic fields with the important difference that the evolution equations of hypermagnetic fields are different from the ones of ordinary magnetic fields. After a swift summary of the properties of hypermagnetic knots (based on [171, 172]), some interesting applications of these hypercharge profiles will be reviewed.

In the gauge $Y_{0}=0, \vec{\nabla} \cdot \vec{Y}=0$, an example of topologically non-trivial configuration of the hypercharge field is the Chern-Simons wave [173, 174, 175

$$
\begin{aligned}
& Y_{x}(z, t)=Y(t) \sin k_{0} z \\
& Y_{y}(z, t)=Y(t) \cos k_{0} z, \\
& Y_{z}(z, t)=0 .
\end{aligned}
$$


This particular configuration is not homogeneous but it describes a hypermagnetic knot with homogeneous helicity and Chern-Simons number density

$$
\begin{aligned}
& \vec{H}_{Y} \cdot \vec{\nabla} \times \vec{H}_{Y}=k_{0} H^{2}(t), \\
& n_{C S}=-\frac{g^{\prime 2}}{32 \pi^{2}} \vec{H}_{Y} \cdot \vec{Y}=\frac{g^{\prime 2}}{32 \pi^{2} k_{0}} H^{2}(t),
\end{aligned}
$$

where $\vec{H}_{Y}=\vec{\nabla} \times \vec{Y}, H(t)=k_{0} Y(t) ; g^{\prime}$ is the $U(1)_{Y}$ coupling.

It is possible to construct hypermagnetic knot configurations with finite energy and helicity which are localized in space and within typical distance scale $L_{s}$. Let us consider in fact the following configuration in spherical coordinates 172

$$
\begin{aligned}
Y_{r}(\mathcal{R}, \theta) & =-\frac{2 B_{0}}{\pi L_{s}} \frac{\cos \theta}{\left[\mathcal{R}^{2}+1\right]^{2}}, \\
Y_{\theta}(\mathcal{R}, \theta) & =\frac{2 B_{0}}{\pi L_{s}} \frac{\sin \theta}{\left[\mathcal{R}^{2}+1\right]^{2}}, \\
Y_{\phi}(\mathcal{R}, \theta) & =-\frac{2 B_{0}}{\pi L_{s}} \frac{n \mathcal{R} \sin \theta}{\left[\mathcal{R}^{2}+1\right]^{2}}
\end{aligned}
$$

where $\mathcal{R}=r / L_{s}$ is the rescaled radius and $B_{0}$ is some dimensionless amplitude and $n$ is just an integer number whose physical interpretation will become clear in a moment. The hypermagnetic field can be easily computed from the previous expression and it is

$$
\begin{aligned}
& H_{r}(\mathcal{R}, \theta)=-\frac{4 B_{0}}{\pi L_{s}^{2}} \frac{n \cos \theta}{\left[\mathcal{R}^{2}+1\right]^{2}}, \\
& H_{\theta}(\mathcal{R}, \theta)=-\frac{4 B_{0}}{\pi L_{s}^{2}} \frac{\mathcal{R}^{2}-1}{\left[\mathcal{R}^{2}+1\right]^{3}} n \sin \theta \\
& H_{\phi}(\mathcal{R}, \theta)=-\frac{8 B_{0}}{\pi L_{s}^{2}} \frac{\mathcal{R} \sin \theta}{\left[\mathcal{R}^{2}+1\right]^{3}} .
\end{aligned}
$$

The poloidal and toroidal components of $\overrightarrow{\mathcal{H}}$ can be usefully expressed as $\vec{H}_{p}=H_{r} \vec{e}_{r}+H_{\theta} \vec{e}_{\theta}$ and $\overrightarrow{\mathcal{H}}_{t}=\mathcal{H}_{\phi} \vec{e}_{\phi}$. The Chern-Simons number is finite and it is given by

$$
N_{C S}=\frac{g^{\prime 2}}{32 \pi^{2}} \int_{V} \overrightarrow{\mathcal{Y}} \cdot \vec{H}_{Y} d^{3} x=\frac{g^{\prime 2}}{32 \pi^{2}} \int_{0}^{\infty} \frac{8 n B_{0}^{2}}{\pi^{2}} \frac{\mathcal{R}^{2} d \mathcal{R}}{\left[\mathcal{R}^{2}+1\right]^{4}}=\frac{g^{\prime 2} n B_{0}^{2}}{32 \pi^{2}}
$$


The total helicity of the configuration can also be computed

$$
\int_{V} \overrightarrow{\mathcal{H}}_{Y} \cdot \vec{\nabla} \times \overrightarrow{\mathcal{H}}_{Y} d^{3} x=\frac{256 B_{0}^{2} n}{\pi L^{2}} \int_{0}^{\infty} \frac{\mathcal{R}^{2} d \mathcal{R}}{\left(1+\mathcal{R}^{2}\right)^{5}}=\frac{5 B_{0}^{2} n}{L_{s}^{2}} .
$$

We can compute also the total energy of the field

$$
E=\frac{1}{2} \int_{V} d^{3} x\left|\overrightarrow{\mathcal{H}}_{Y}\right|^{2}=\frac{B_{0}^{2}}{2 L_{s}}\left(n^{2}+1\right)
$$

and we discover that it is proportional to $n^{2}$. This means that one way of increasing the total energy of the field is to increase the number of knots and twists in the flux lines.

This type of configurations can be also obtained by projecting a nonAbelian SU(2) (vacuum) gauge field on a fixed electromagnetic direction [176] 24. The resulting profile of the knot depends upon an arbitrary function of the radial distance.

These configurations have been also studied in [178, 179]. In particular, in [179, the relaxation of HK has been investigated with a technique different from the one employed in [171, 172] but with similar results. The problem of scattering of fermions in the background of hypermagnetic fields has been also studied in [180, 181].

Hypermagnetic knots with large correlation scale can be also generated dynamically provided an unknotted hypermagnetic background is already present.

Let us assume that dynamical pseudoscalar particles are evolving in the background geometry given by Eq. (5.1). The pseudoscalars are not a source of the background (i.e. they do not affect the time evolution of the scale factor) but, nonetheless, they evolve according to their specific dynamics and can excite other degrees of freedom.

The action describing the interaction of a dynamical pseudoscalar with hypercharge fields can be written as

$$
S=\int d^{4} x \sqrt{-g}\left[\frac{1}{2} g^{\alpha \beta} \partial_{\alpha} \psi \partial_{\beta} \psi-V(\psi)-\frac{1}{4} Y_{\alpha \beta} Y^{\alpha \beta}+c \frac{\psi}{4 M} Y_{\alpha \beta} \tilde{Y}^{\alpha \beta}\right] .
$$

\footnotetext{
${ }^{24}$ In order to interpret these solutions it is very interesting to make use of the Clebsh decomposition. The implications of this decomposition (beyond the hydrodynamical context, where it was originally discovered) have been recently discussed (see [177] and references therein).
} 
This action is quite generic. In the case $V(\psi)=\left(m^{2} / 2\right) \psi^{2}$ Eq. (5.120) is nothing but the curved space generalization of the model usually employed in direct searches of axionic particles [182, 183, 184. The constant in front of the anomaly is a model-dependent factor. For example, in the case of axionic particles, for large temperatures $T \geq m_{W}$, the Abelian gauge fields present in Eq. (5.120) will be hypercharge fields and $c=c_{\psi Y} \alpha^{\prime} /(2 \pi)$ where $\alpha^{\prime}=g^{\prime 2} / 4 \pi$ and $c_{\psi Y}$ is a numerical factor of order 1 which can be computed (in a specific axion scenario) by knowing the Peccei-Quinn charges of all the fermions present in the model [185, 186. For small temperatures $T \leq m_{W}$ we have that the Abelian fields present in the action (5.120) will coincide with ordinary electromagnetic fields and $c=c_{\psi \gamma} \alpha_{\mathrm{em}} / 2 \pi$ where $\alpha_{\mathrm{em}}$ is the fine structure constant and $c_{\psi \gamma}$ is again a numerical factor.

The coupled system of equations describing the evolution of the pseudoscalars and of the Abelian gauge fields can be easily derived by varying the action with respect to $\psi$ and $Y_{\mu}$,

$$
\begin{aligned}
& \frac{1}{\sqrt{-g}} \partial_{\mu}\left[\sqrt{-g} g^{\mu \nu} \partial_{\nu} \psi\right]+\frac{\partial V}{\partial \psi}=\frac{c}{4 M} Y_{\alpha \beta} \tilde{Y}^{\alpha \beta}, \\
& \nabla_{\mu} Y^{\mu \nu}=\frac{c}{M} \nabla_{\mu} \psi \tilde{Y}^{\mu \nu}, \quad \nabla_{\mu} \tilde{Y}^{\mu \nu}=0,
\end{aligned}
$$

where,

$$
\nabla_{\mu} Y^{\mu \nu}=\frac{1}{\sqrt{-g}} \partial_{\mu}\left[\sqrt{-g} Y^{\mu \nu}\right], \quad \nabla_{\mu} \tilde{Y}^{\mu \nu}=\frac{1}{\sqrt{-g}} \partial_{\mu}\left[\sqrt{-g} \tilde{Y}^{\mu \nu}\right],
$$

are the usual covariant derivatives defined from the background FRW metric Eqs. (5.121) can be written in terms of the physical gauge fields

$$
\begin{aligned}
& \psi^{\prime \prime}+2 \mathcal{H} \psi^{\prime}-\nabla^{2} \psi+a^{2} \frac{\partial V}{\partial \psi}=-\frac{1}{a^{2}} \frac{c}{M} \overrightarrow{\mathcal{E}}_{Y} \cdot \overrightarrow{\mathcal{H}}_{Y}, \\
& \vec{\nabla} \cdot \overrightarrow{\mathcal{H}}_{Y}=0, \quad \vec{\nabla} \times \overrightarrow{\mathcal{E}}_{Y}+\overrightarrow{\mathcal{H}}_{Y}^{\prime}=0, \quad \vec{\nabla} \cdot \overrightarrow{\mathcal{E}}_{Y}=\frac{c}{M} \vec{\nabla} \psi \cdot \overrightarrow{\mathcal{H}}_{Y}, \\
& \vec{\nabla} \times \overrightarrow{\mathcal{H}}_{Y}=\overrightarrow{\mathcal{E}}_{Y}^{\prime}-\frac{c}{M}\left[\psi^{\prime} \overrightarrow{\mathcal{H}}_{Y}+\vec{\nabla} \psi \times \overrightarrow{\mathcal{E}}_{Y}\right] .
\end{aligned}
$$

We want now to study the amplification of gauge field fluctuations induced by the time evolution of $\psi$. Then, the evolution equation for the hypermagnetic fluctuations $\overrightarrow{\mathcal{H}}_{Y}$ can be obtained by linearizing Eqs. (5.123). We will assume that any background gauge field is absent. In the linearisation procedure we will also assume that the pseudoscalar field can be treated 
as completely homogeneous (i.e. $|\vec{\nabla} \psi| \ll \psi^{\prime}$ ). This seems to be natural if, prior to the radiation dominated epoch, an inflationary phase diluted the gradients of the pseudoscalar.

In this approximation, the result of the linearization can be simply written in terms of the vector potentials in the gauge $Y^{0}=0$ and $\vec{\nabla} \cdot \vec{Y}=0$ :

$$
\begin{aligned}
& \overrightarrow{\mathcal{Y}}^{\prime \prime}-\nabla^{2} \overrightarrow{\mathcal{Y}}+\frac{c}{M} \psi^{\prime} \vec{\nabla} \times \overrightarrow{\mathcal{Y}}=0, \\
& \ddot{\psi}+3 H \dot{\psi}+\frac{\partial V}{\partial \psi}=0
\end{aligned}
$$

By combining the evolution equations for the gauge fields we can find a decoupled evolution equation for $\overrightarrow{\mathcal{H}}_{Y}$,

$$
\overrightarrow{\mathcal{H}}_{Y}^{\prime \prime}-\nabla^{2} \overrightarrow{\mathcal{H}}_{Y}+\frac{c}{M} \psi^{\prime} \vec{\nabla} \times \overrightarrow{\mathcal{H}}_{Y}=0
$$

From this equation is already apparent that the pseudo-scalar vertex induces an interaction in the two physical polarizations of the hypermagnetic field. Giving initial conditions which are such that $\overrightarrow{\mathcal{H}}_{Y} \neq 0$ with $\overrightarrow{\mathcal{H}}_{Y} \cdot \vec{\nabla} \times \overrightarrow{\mathcal{H}}_{Y}=0$ a profile with $\overrightarrow{\mathcal{H}}_{Y} \cdot \vec{\nabla} \times \overrightarrow{\mathcal{H}}_{Y} \neq 0$ can be generated provided $\psi^{\prime} \neq 0$.

\section{Two approaches to the Origin}

Since the flux is conserved the ratio between the magnetic energy density, $\rho_{\mathrm{B}}(L, \eta)$ and the energy density sitting in radiation, $\rho_{\gamma}(\eta)$ is almost constant and therefore, in terms of this quantity (which is only scale dependent but not time dependent), the most simplistic dynamo requirement can be rephrased (see Section 3) as

$$
r_{\mathrm{B}}(L)=\frac{\rho_{\mathrm{B}}(L, \eta)}{\rho_{\gamma}(\eta)} \geq 10^{-34}, \quad L \sim 1 \mathrm{Mpc},
$$

to be compared with the value $r_{\mathrm{B}} \sim 10^{-8}$ which would lead to the galactic magnetic field only thanks to the collapse and without the need of dynamo action (this would be the case when the magnetic field is fully primordial). Notice that Eq. (6.1) assumes that the magnetic flux is exactly frozen into the plasma element. 
As previously pointed out during the discussion of the dynamo mechanism, the requirement expressed by Eq. (6.1) is unrealistic: it would correspond to thirty e-folds of amplification during galactic rotation and perfect flux freezing during the collapse of the protogalaxy. In a more realistic situation situation, taking into account the effectively achievable amplification of the dynamo action the requirement would rather be, prior to collapse,

$$
r_{\mathrm{B}}(L)=\frac{\rho_{\mathrm{B}}(L, \eta)}{\rho_{\gamma}(\eta)} \geq 10^{-23}, \quad L \sim 1 \mathrm{Mpc} .
$$

In spite of the richness of the theoretical models, the mechanisms for magnetic field generation can be divided, broadly speaking, into two categories: astrophysical and cosmological. The cosmological mechanisms can be divided, in turn, into causal mechanisms (where the magnetic seeds are produced at a given time inside the horizon) and inflationary mechanisms where correlations in the magnetic field are produced outside the horizon.

\subsection{Inhomogeneous MHD equations}

The first attempts in this direction have been made by Biermann [187] and Harrison [189, 190, 191]. The Biermann battery mechanism is easy to understand from the form of the generalized Ohm law discussed in Eq. (4.17). MHD equations are linear and homogeneous in the magnetic field intensities. The idea is then to look for a natural source term which seemed to be provided by the thermoelectric current already introduced in the context of the generalized Ohm law . Consider, indeed, Eq. (4.17) in the approximation where the Hall term is neglected (the argument can be also generalized to the case of non vanishing Hall term). Then, the Ohmic electric field will not be the one simply obtained in resistive MHD but it will get a contribution from the thermoelectric term:

$$
\vec{E}=-\vec{v} \times \vec{B}+\frac{\vec{\nabla} \times \vec{B}}{4 \pi \sigma}-\frac{1}{e n_{\mathrm{e}}} \vec{\nabla} P_{\mathrm{e}} .
$$

Using the other MHD equations in the incompressible approximation the new form of the magnetic diffusivity equation can be derived:

$$
\frac{\partial \vec{B}}{\partial t}-\vec{\nabla} \times(\vec{v} \times \vec{B})-\frac{1}{4 \pi \sigma} \nabla^{2} \vec{B}=-\frac{1}{e n_{\mathrm{e}}^{2}} \vec{\nabla} n_{\mathrm{e}} \times \vec{\nabla} P_{\mathrm{e}}
$$


The magnetic diffusivity equation has now a source terms which does not vanish provided the gradients of the charge density of the electrons is not parallel to the pressure gradients. As a consequence of the presence of the source term, the magnetic field will grow linearly in time until the thermoelectric source term is comparable with the dynamo term. The estimate of the magnetic field intensity prior to the onset of galactic rotation will then be

$$
|\vec{B}| \simeq \frac{t}{e n_{\mathrm{e}}^{2}}\left|\vec{\nabla} n_{\mathrm{e}} \times \vec{\nabla} P_{\mathrm{e}}\right|,
$$

leading to magnetic fields $\mathcal{O}\left(10^{-20}\right) \mathrm{G}$ over typical scales of the order of 10 kpc (see also 188 for a recent analysis in a similar framework).

In a complementary perspective, Harrison 189, 190, 191] discussed the possibility that the pressure gradients are strictly vanishing, i.e. $\vec{\nabla} p_{e}=$ 0 . This is a reasonable assumption, for instance, in a radiation-dominated stage of expansion where pressure gradients are expected to be negligible by the global homogeneity of the background. In this situation, an evolution equation for the vorticity can be derived

$$
\frac{\partial}{\partial \eta}\left(a^{2} \vec{\omega}+\frac{e}{m_{\mathrm{p}}} \overrightarrow{\mathcal{B}}\right)=\frac{e}{4 \pi \sigma m_{\mathrm{p}}} \nabla^{2} \overrightarrow{\mathcal{B}},
$$

where $\omega=\vec{\nabla} \times \vec{v}$ and $m_{\mathrm{p}}$ is the ion mass. If we now postulate that some vorticity was present prior to decoupling, then Eq. (6.6) can be solved and the magnetic field can be related to the initial vorticity as

$$
\vec{B} \sim-\frac{m_{\mathrm{p}}}{e} \vec{\omega}_{\mathrm{i}}\left(\frac{a_{i}}{a}\right)^{2} .
$$

It is clear that if the estimate of the vorticity is made prior to equality (as originally suggested by Harrison [189]) of after decoupling as also suggested, a bit later 192] can change even by two orders of magnitude. Prior to equality $\mid \vec{\omega}(t) \simeq 0.1 / t$ and, therefore, $\left|\vec{B}_{\text {eq }}\right| \sim 10^{-21} \mathrm{G}$. If a similar estimate is made after decoupling the typical value of the generated magnetic field is of the order of $10^{-18} \mathrm{G}$. So, in this context, the problem of the origin of magnetic fields is circumvented by postulating an appropriate form of vorticity whose origin must be explained.

The idea was employed, later on, in the context of the physics of topological defects. Vachaspati and Vilenkin [193 have suggested that cosmic strings with small scale structure may be a source of the wanted vorticity. 
The argument is, in short, that since matter flow in baryonic wakes is turbulent, velocity gradients will be induced in the flow by the small-scale wiggles which produced the required vorticity. Furthermore, dynamical friction between cosmic strings and matter may provide a further source of vorticity 194. There have been also studies trying to generate large-scale magnetic fields in the context of superconducting cosmic strings (see, for instance, [195] and references therein).

Recently the possible generation of large-scale magnetic fields prior to hydrogen recombination has been discussed in 196, 197. The vorticity required in order to produce the magnetic fields is generated, according to [196], by the photon diffusion at second order in the temperature fluctuations. In a similar perspective Hogan [198] got much less optimistic estimates which, according to [196, 197], should be attributed to different approximation schemes employed in the analysis.

\subsection{Inflationary mechanisms}

Different astrophysical objects, of different physical sizes, have comparable magnetic fields. This coincidence is hard to explain in the context of causal mechanisms of generation. The analogy with structure formation, already presented in the Introduction, is here useful. In the late seventies, prior to the formulation of the inflationary paradigm, the initial conditions for the density contrast were taken as primordial input. Later on, in the context of inflationary models, the primordial spectrum of curvature and density fluctuations could be calculated.

During inflation, in fact, fields of various spin are present and they can be excited by the dynamical evolution of the geometry. In the context of inflationary models of particular relevance are the scalar and tensor fluctuations of the geometry, corresponding respectively, to fluctuations of the scalar curvature and to gravitational waves. Gravitational waves and curvature perturbations obey evolution equations which are not invariant under Weyl rescaling of the four-dimensional metric. Then the quantum mechanical fluctuations in the corresponding fields will simply be amplified by the evolution of the background geometry.

It is interesting to speculate that large scale magnetic fields could be produced thanks to a similar mechanism. The major obstruction to this type of models is that gauge fields are not amplified thanks to the evolution of the background geometry the reason being that the evolution equations of 
gauge fields are invariant under Weyl rescaling of the metric.

When conformal invariance is broken, by some means, one is often led to estimate the amplitude of gauge field fluctuations arising as a result of the breaking. Clearly the detailed amount of amplified gauge fields will be specific of the particular model. In the following various ways of breaking conformal invariance will be listed without getting through the details of the calculations. Before doing so it is anyway instructive to introduce some general considerations stressing the analogy between the production of magnetic inhomogeneities and the production of gravitational inhomogeneities whose late time evolution leads the anisotropies in the CMB.

The tensor modes of the geometry are described by a rank-two (transverse and traceless) tensor in three spatial dimensions ${ }^{25}$, i.e.

$$
d s^{2}=a^{2}(\eta)\left[d \eta^{2}-\left(\delta_{i j}+h_{i j}\right) d x^{i} d x^{j}\right], \quad h_{i}^{i}=\partial_{i} h_{j}^{i}=0,
$$

obeying the equation, in Fourier space,

$$
\mu_{k}^{\prime \prime}+\left[k^{2}-\frac{a^{\prime \prime}}{a}\right] \mu_{k}=0,
$$

where $\mu_{k}=a h_{k}$ and $h_{k}$ is the Fourier mode of each polarization. In this equation the "pump field" is simply given by the scale factor. When $k^{2} \gg$ $\left|a^{\prime \prime} / a\right|$ the mode is said to be adiabatically damped: in fact, in this regime, $\left|h_{k}\right| \simeq a^{-1}$, i.e. decreasing in an expanding Universe. In the opposite regime, i.e. $k^{2} \ll\left|a^{\prime \prime} / a\right|$ the mode is super-adiabatically amplified. In fact, during a de Sitter or quasi-de Sitter $\mu_{k} \sim a(\eta)$ and $h_{k}$ is constant. Hence, for the whole time the given mode spends under the "potential barrier" of Eq. (6.9), it is amplified.

A similar equation holds for the canonical normal mode for the scalar fluctuations of the geometry, i.e.

$$
v_{k}^{\prime \prime}+\left[k^{2}-\frac{z^{\prime \prime}}{z}\right] v_{k}=0 .
$$

The variable $v_{k}$ is defined as

$$
v_{k}=a \delta \varphi_{k}+z \psi_{k}, \quad \quad \mathcal{R}_{k}=-\frac{v_{k}}{z},
$$

\footnotetext{
${ }^{25}$ The theory of cosmological perturbations is assumed in the following considerations. Comprehensive accounts of the relevant topics can be found in [199, 200] (see also 201]).
} 
where $\delta \varphi_{k}$ is the fluctuation of the inflaton, $\mathcal{R}_{k}$ is the curvature perturbation and $\psi$ is the scalar fluctuation of the geometry in the conformally Newtonian gauge [199, 200. If the inflaton has an exponential potential $z(\eta) \simeq a(\eta)$.

In the case of gauge fields, each of the two polarization of the appropriately rescaled vector potential obeys, the following equation

$$
\mathcal{A}_{k}^{\prime \prime}+\left[k^{2}-g\left(g^{-1}\right)^{\prime \prime}\right] \mathcal{A}_{k}=0
$$

In specific models, $g(\eta)$ may be associated with the gauge coupling. However $g^{-1}(\eta)$ can also be viewed as a generic pump field arising as a result of the breaking of conformal invariance. The time dependence of the potential term is rather common to different models: it goes to zero for $|\eta| \rightarrow \infty$ as $\left(\nu^{2}-\right.$ $1 / 4) \eta^{-2}$. The numerical coefficient appearing in the potential determines the strength and spectrum of the amplified gauge fields whose subsequent evolution has to be however computed at finite conductivity. In Fig. 66 the

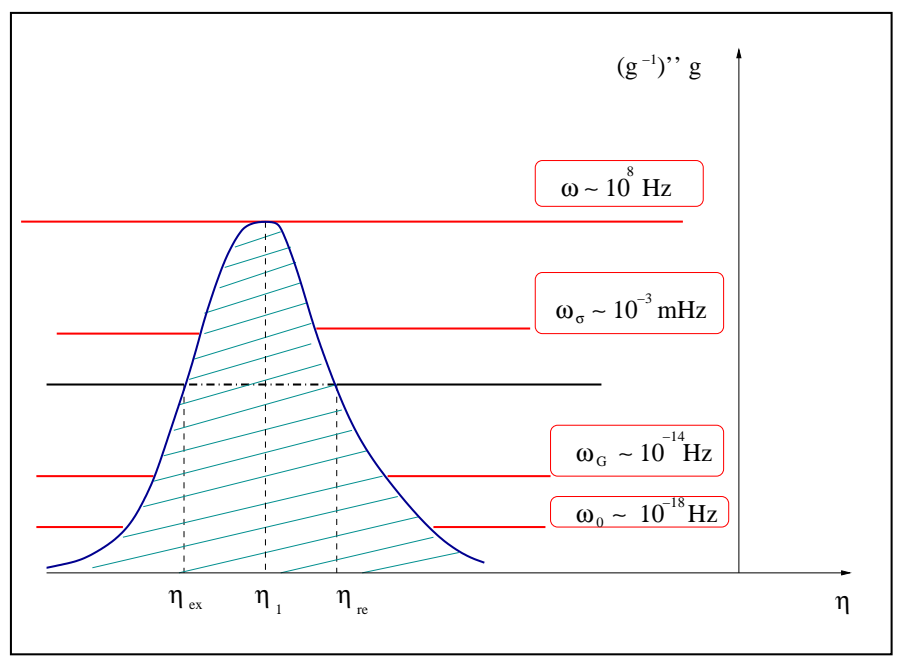

Figure 6: The effective potential appearing in Eq. (6.12) is illustrated in general terms. In this example the pump field leading to amplification of electromagnetic quantum fluctuations is assumed to be constant prior to the onset of BBN. On the vertical axis few relevant physical frequencies (to be compared with the height of the potential) have been reported.

typical form of the potential term appearing in Eq. (6.12) is illustrated. Different comoving frequencies go under the barrier at different times and 
the amount of amplification is roughly proportional to the time spent under the barrier. Clearly, given the generic form of the barrier, smaller frequencies are more amplified than the frequencies comparable with the height of the barrier at $\eta_{1}$. In Fig. 6 the explicit numerical value of the height of the barrier corresponds to a (present) frequency of $10^{8} \mathrm{~Hz}$ which can be realized if the pump field goes to a constant right after the end of a conventional inflationary phase followed by a radiation-dominated stage of expansion.

In the case of gravitational waves, and, with some caveats, in the case of scalar metric fluctuations, the "potential barriers" appearing, respectively, in Eqs. (6.9) and (6.10) may be related with the inverse Hubble radius. Hence, in the case of metric fluctuations, a mode which is under the barrier is also, with a swift terminology, outside the horizon (see Fig. 7). This is the reason why, following the conventional nomenclature, in Fig. 6] the moment when a given scale gets under the barrier has been denoted by $\eta_{\mathrm{ex}}$ (i.e. horizon exit). According to the same convention, the moment when a given scale gets out the potential barrier is labeled by $\eta_{\text {re }}$ (i.e. horizon re-entry). In Fig. 6 few

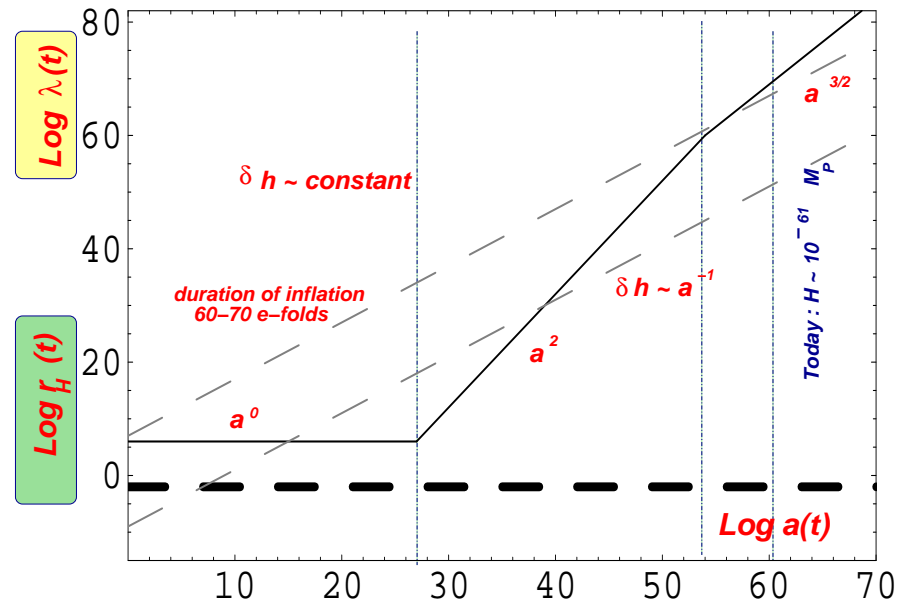

Figure 7: The evolution of a given physical wavelength is illustrated in the case of an inflationary model with minimal duration. The region above the full curve (denoting the Hubble radius $r_{H}$ ) $=H^{-1}$ ) corresponds, for the tensor and scalar modes of the geometry, to the region where a given comoving wavenumber $k$ is below the "potential barrier" appearing in Eqs. (6.9) and (6.10).

relevant frequencies have been compared with the height of the barrier. Con- 
sider, for instance, $\omega_{\mathrm{G}}$ i.e. the typical scale of gravitational collapse which is of the order of $1 \mathrm{Mpc}$, i.e. $10^{-14} \mathrm{~Hz}$. In Fig. 6 the physical frequency has been directly reported. Another interesting frequency is $\omega_{\sigma} \sim \mathrm{mHz}$ corresponding to the present value of the magnetic diffusivity momentum. The amplification caused by the parametric amplification of the vacuum fluctuations can be computed by solving Eq. (6.12) in the different regimes.

$$
\begin{array}{ll}
\mathcal{A}_{k} & =\frac{e^{-i k \eta}}{\sqrt{k}}, \quad \eta<\eta_{\text {ex }}, \\
\mathcal{A}_{k}=g^{-1}(\eta)\left[C_{k}+D_{k} \int^{\eta} d \eta^{\prime} g^{2}\left(\eta^{\prime}\right)\right], & \eta_{e x}<\eta<\eta_{r e} \\
\mathcal{A}_{k}=\frac{1}{\sqrt{k}}\left[c_{+}(k) e^{-i k \eta}+c_{-}(k) e^{i k \eta}\right], & \eta>\eta_{r e},
\end{array}
$$

where $\left(C_{k}, D_{k}\right.$, and $c_{ \pm}(k)$ are integrations constants).

The mixing coefficients $c_{ \pm}(k)$, determining the parametric amplification of a mode $k^{2}<\left|V\left(\eta_{1}\right)\right|$, computed by matching these various branches of the solution reported in Eq. (6.13). One finds:

$$
\begin{aligned}
& 2 i k e^{-i k\left(\eta_{e x} \mp \eta_{r e}\right)} c_{ \pm}(k)=\mp \frac{g_{e x}}{g_{r e}}\left(-\frac{g_{r e}{ }^{\prime}}{g_{r e}} \mp i k\right) \pm \frac{g_{r e}}{g_{e x}}\left(-\frac{g_{e x}{ }^{\prime}}{g_{e x}}+i k\right) \\
\pm & \frac{1}{g_{e x} g_{r e}}\left(-\frac{g_{e x}{ }^{\prime}}{g_{e x}}+i k\right)\left(-\frac{g_{r e}{ }^{\prime}}{g_{r e}} \mp i k\right) \int_{\eta_{e x}}^{\eta_{r e}} g^{2} d \eta .
\end{aligned}
$$

Similar calculations can be performed in order to obtain the spectrum of scalar and tensor fluctuations of the geometry [204, 205, 206].

Suppose now to make a simple estimate. Assume, for instance, that $g$ is evolving prior to $\eta_{1}$ according to the dynamics dictated by a given model. Suppose also that after $\eta_{1}$ the Universe is suddenly dominated by radiation, and $g^{\prime} \sim 0$ for $\eta>\eta_{1}$. In this situation all the modes reenter during radiation and the amplification will be roughly given, to leading order by

$$
\left|c_{-}\right| \simeq \frac{g_{r e}}{g_{e x}} .
$$

If the function $g(\eta)$ is identified with the evolving gauge coupling this result suggest that in order to have large amplification, $g(\eta)$ has to grow from smaller to larger values. This is what happens, for instance, in the case of pre-big bang models where $g \sim e^{\varphi / 2}$ and $\varphi$ is the for-dimensional dilaton field 123. 
Since we ought to estimate the amplification of an initial quantum mechanical fluctuations, a fully quantum mechanical treatment is certainly appropriate also in view of the discussion of the correlation properties of the obtained fluctuations. This analysis has been performed in 207] where the squeezing properties of the obtained photons have also been discussed.

The perturbed effective Lagrangian density

$\mathcal{L}(\vec{x}, \eta)=\frac{1}{2} \sum_{\alpha}\left[\mathcal{A}_{\alpha}^{\prime 2}+2 \frac{g^{\prime}}{g} \mathcal{A}_{\alpha}^{\prime} \mathcal{A}_{\alpha}+\left(\frac{g^{\prime}}{g}\right)^{2} \mathcal{A}_{\alpha}^{2}-\left(\partial_{i} \mathcal{A}_{\alpha}\right)^{2}\right], \quad L(\eta)=\int d^{3} x \mathcal{L}(\vec{x}, \eta)$,

describes the evolution of the two $(\alpha=\otimes, \oplus)$ transverse degrees of freedom defined by the Coulomb gauge condition $A_{0}=0$ and $\vec{\nabla} \cdot \vec{A}=0$ (the prime denotes differentiation with respect to conformal time). The fields $A_{\alpha}=g \mathcal{A}_{\alpha}$ have kinetic terms with canonical normalization and the time evolution given in Eq. (6.12) stems from the Euler-Lagrange equations derived from Eq. (6.16). By functionally deriving the the action the canonically conjugated momenta can be obtained leading to the Hamiltonian density and to the associated Hamiltonian

$$
\begin{aligned}
& \mathcal{H}(\vec{x}, \eta)=\frac{1}{2} \sum_{\alpha}\left[\pi_{\alpha}^{2}+\left(\partial_{i} \mathcal{A}_{\alpha}\right)^{2}-2 \frac{g^{\prime}}{g} \mathcal{A}_{\alpha} \pi_{\alpha}\right], \\
& H(\eta)=\int d^{3} x \mathcal{H}(\vec{x}) .
\end{aligned}
$$

The operators corresponding to the classical polarizations appearing in the Hamiltonian density

$$
\begin{aligned}
& \hat{\mathcal{A}}_{\alpha}(\vec{x}, \eta)=\int d^{3} k \frac{1}{(2 \pi)^{3 / 2}} \hat{\mathcal{A}}_{\alpha}(k, \eta) e^{i \vec{k} \cdot \vec{x}}, \\
& \hat{\mathcal{A}}_{\alpha}(k, \eta)=\frac{1}{\sqrt{2 k}}\left[\hat{a}_{k, \alpha}(\eta)+\hat{a}_{-k, \alpha}^{\dagger}(\eta)\right], \\
& \hat{\pi}_{\alpha}(\vec{x}, \eta)=\int d^{3} k \frac{1}{(2 \pi)^{3 / 2}} \hat{\pi}_{\alpha}(k, \eta) e^{i \vec{k} \cdot \vec{x}}, \\
& \hat{\pi}_{\alpha}(k, \eta)=i \sqrt{\frac{k}{2}}\left[\hat{a}_{k, \alpha}(\eta)-\hat{a}_{-k, \alpha}^{\dagger}(\eta)\right],
\end{aligned}
$$

obey canonical commutation relations and the associated creation and annihilation operators satisfy $\left[\hat{a}_{k, \alpha}, \hat{a}_{p, \beta}^{\dagger}\right]=\delta_{\alpha \beta} \delta^{(3)}(\vec{k}-\vec{p})$.

The (two-modes) Hamiltonian contains a free part and the effect of the variation of the coupling constant is encoded in the (Hermitian) interaction 
term which is quadratic in the creation and annihilation operators whose evolution equations, read, in the Heisenberg picture

$$
\begin{aligned}
& \frac{d \hat{a}_{k, \alpha}}{d \eta}=-i k \hat{a}_{k, \alpha}-\frac{g^{\prime}}{g} \hat{a}_{-k, \alpha}^{\dagger}, \\
& \frac{d \hat{a}_{k, \alpha}^{\dagger}}{d \eta}=i k \hat{a}_{k, \alpha}^{\dagger}-\frac{g^{\prime}}{g} \hat{a}_{-k, \alpha} .
\end{aligned}
$$

The general solution of the previous system of equations can be written in terms of a Bogoliubov-Valatin transformation,

$$
\begin{aligned}
& \hat{a}_{k, \alpha}(\eta)=\mu_{k, \alpha}(\eta) \hat{b}_{k, \alpha}+\nu_{k, \alpha}(\eta) \hat{b}_{-k, \alpha}^{\dagger} \\
& \hat{a}_{k, \alpha}^{\dagger}(\eta)=\mu_{k, \alpha}^{*}(\eta) \hat{b}_{k, \alpha}^{\dagger}+\nu_{k, \alpha}^{*}(\eta) \hat{b}_{-k, \alpha}
\end{aligned}
$$

where $\hat{a}_{k, \alpha}(0)=\hat{b}_{k, \alpha}$ and $\hat{a}_{-k, \alpha}(0)=\hat{b}_{-k, \alpha}$. Notice that the Bogoliubov coefficients are the quantum analog of the mixing coefficients discussed in the semiclassical approach to the problem.

Unitarity requires that the two complex functions $\mu_{k}(\eta)$ and $\nu_{k}(\eta)$ are subjected to the condition $\left|\mu_{k}(\eta)\right|^{2}-\left|\nu_{k}(\eta)\right|^{2}=1$ which also implies that $\mu_{k}(\eta)$ and $\nu_{k}(\eta)$ can be parameterized in terms of one real amplitude and two real phases

$$
\mu_{k}=e^{i \theta_{k}} \cosh r_{k}, \quad \nu_{k}=e^{i\left(2 \phi_{k}-\theta_{k}\right)} \sinh r_{k},
$$

( $r$ is sometimes called squeezing parameter and $\phi_{k}$ is the squeezing phase; from now on we will drop the subscript labeling each polarization if not strictly necessary). The total number of produced photons

$$
\left\langle 0_{-k} 0_{k}\left|\hat{a}_{k}^{\dagger}(\eta) \hat{a}_{k}(\eta)+\hat{a}_{-k}^{\dagger} \hat{a}_{-k}\right| 0_{k} 0_{-k}\right\rangle=2 \bar{n}_{k} .
$$

is expressed in terms of $\bar{n}_{k}=\sinh ^{2} r_{k}$, i.e. the mean number of produced photon pairs in the mode $k$. Inserting Eqs. (6.20) (6.22) into Eqs. (6.19) we can derive a closed system involving only the $\bar{n}_{k}$ and the related phases:

$$
\begin{aligned}
\frac{d \bar{n}_{k}}{d \eta} & =-2 f\left(\bar{n}_{k}\right) \frac{g^{\prime}}{g} \cos 2 \phi_{k}, \\
\frac{d \theta_{k}}{d \eta} & =-k+\frac{g^{\prime}}{g} \frac{\bar{n}_{k}}{f\left(\bar{n}_{k}\right)} \sin 2 \phi_{k}, \\
\frac{d \phi_{k}}{d \eta} & =-k+\frac{g^{\prime}}{g} \frac{d f\left(\bar{n}_{k}\right)}{d \bar{n}_{k}} \sin 2 \phi_{k},
\end{aligned}
$$


where $f\left(\bar{n}_{k}\right)=\sqrt{\bar{n}_{k}\left(\bar{n}_{k}+1\right)}$.

In quantum optics [208, 209] the coherence properties of light fields have been a subject of intensive investigations for nearly half a century. In the present context the multiparticle states described so fare are nothing but squeezed states of the electromagnetic field [208, 209]. In fact, up to now the Heisenberg description has been adopted. In the Schrödinger picture the quantum mechanical states obtained as a result of the time evolution are exactly squeezed vacuum states [208, 209].

Magnetic fields over galactic scales have typical frequency of the order $10^{-14}-10^{-15} \mathrm{~Hz}$ which clearly fall well outside the optical range. Thus, the analogy with quantum optics is only technical. The same quantum optical analogy has been successfully exploited in particle [210] and heavy-ions physics [211] of pion correlations (in order to measure the size of the strongly interacting region) and in the phenomenological analysis of hadronic multiplicity distributions.

The interference between the amplitudes of the magnetic fields (Young interferometry [212], in a quantum optical language) estimates the first order coherence of the magnetic background at different spatial locations making use of the two-point correlation function whose trace over the physical polarizations and for coincidental spatial locations is related to the magnetic energy density. Eqs. (6.23) -6.25) can be solved once the pump field is specified but general expressions can be also obtained [207].

\subsubsection{Conventional inflationary models}

In conventional inflationary models it is very difficult to produce large scale magnetic fields with phenomenologically relevant strength. This potential difficulty has been scrutinized in various investigations [202, 213, 214, 215].

Turner and Widrow 213] listed a series of field theory models in de Sitter space with the purpose of finding a natural way of breaking conformal invariance. The first suggestion was that conformal invariance may be broken, at an effective level, through the coupling of photons to the geometry [216]. Typically, the breaking of conformal invariance occurs through products of gauge-field strengths and curvature tensors, i.e.

$$
\frac{1}{m^{2}} F_{\mu \nu} F_{\alpha \beta} R^{\mu \nu \alpha \beta}, \quad \frac{1}{m^{2}} R_{\mu \nu} F^{\mu \beta} F^{\nu \alpha} g_{\alpha \beta}, \quad \frac{1}{m^{2}} F_{\alpha \beta} F^{\alpha \beta} R
$$

where $m$ is the appropriate mass scale; $R_{\mu \nu \alpha \beta}$ and $R_{\mu \nu}$ are the Riemann and Ricci tensors and $R$ is the Ricci scalar. If the evolution of gauge fields is 
studied during a phase of de Sitter (or quasi-de Sittter) expansion, then the amplification of the vacuum fluctuations induced by the couplings listed in Eq. 6.26) is minute. The price in order to get large amplification should be, according to 213, an explicit breaking of gauge-invariance by direct coupling of the vector potential to the Ricci tensor or to the Ricci scalar, i.e.

$$
R A_{\mu} A^{\mu}, \quad R_{\mu \nu} A^{\mu} A^{\nu}
$$

In 213] two other different models were proposed (but not scrutinized in detail) namely scalar electrodynamics and the axionic coupling to the Abelian field strength.

Dolgov [215] considered the possible breaking of conformal invariance due to the trace anomaly. The idea is that the conformal invariance of gauge fields is broken by the triangle diagram where two photons in the external lines couple to the graviton through a loop of fermions. The local contribution to the effective action leads to the vertex $(\sqrt{-g})^{1+\epsilon} F_{\alpha \beta} F^{\alpha \beta}$ where $\epsilon$ is a numerical coefficient depending upon the number of scalars and fermions present in the theory. The evolution equation for the gauge fields, can be written, in Fourier space, as

$$
\mathcal{A}_{k}^{\prime \prime}+\frac{\epsilon}{8} \mathcal{H} \mathcal{A}_{k}^{\prime}+k^{2} \mathcal{A}_{k}=0
$$

and it can be shown that only if $\epsilon>0$ the gauge fields are amplified. Furthermore, only is $\epsilon \sim 8$ substantial amplification of gauge fields is possible.

In a series of papers [217, 218, 219] the possible effect of the axionic coupling to the amplification of gauge fields has been investigated. The idea is here that conformal invariance is broken through the explicit coupling of a pseudo-scalar field to the gauge field (see Section 5), i.e.

$$
\sqrt{-g} c_{\psi \gamma} \alpha_{\mathrm{em}} \frac{\psi}{8 \pi M} F_{\alpha \beta} \tilde{F}^{\alpha \beta}
$$

where $\tilde{F}^{\alpha \beta}$ is the dual field strength and where $c_{\psi \gamma}$ is a numerical factor of order one. Consider now the case of a standard pseudoscalar potential, for instance $m^{2} \psi^{2}$, evolving in a de Sitter (or quasi-de Sitter space-time). It can be shown, rather generically, that the vertex given in Eq. (6.29) leads to negligible amplification at large length-scales. The coupled system of evolution equations to be solved in order to get the amplified field is similar 
to Eqs. (5.123) already introduced in the duscussion of hypermagnetic fields

$$
\begin{aligned}
& \overrightarrow{\mathcal{B}^{\prime \prime}}-\nabla^{2} \overrightarrow{\mathcal{B}}-\frac{\alpha_{\mathrm{em}}}{2 \pi M} \psi^{\prime} \vec{\nabla} \times \overrightarrow{\mathcal{B}}=0, \\
& \psi^{\prime \prime}+2 \mathcal{H} \psi^{\prime}+m^{2} a^{2} \psi=0 .
\end{aligned}
$$

From Eq. (6.30), there is a maximally amplified physical frequency

$$
\omega_{\max } \simeq \frac{\alpha_{\mathrm{em}}}{2 \pi M} \dot{\psi}_{\max } \simeq \frac{\alpha_{\mathrm{em}}}{2 \pi} m
$$

where the second equality follows from $\psi \sim a^{-3 / 2} M \cos m t$ (i.e. $\dot{\psi}_{\max } \sim m M$ ). The amplification for $\omega \sim \omega_{\max }$ is of the order of $\exp \left[m \alpha_{\mathrm{em}} /(2 \pi H)\right]$ where $H$ is the Hubble parameter during the de Sitter phase of expansion. From the above expressions one can argue that the modes which are substantially amplifed are the ones for which $\omega_{\max } \gg H$. The modes interesting for the large-scale magnetic fields are the ones which are in the opposite range, i.e. $\omega_{\max } \ll H$. Clearly, by lowering the curvature scale of the problem the produced seeds may be larger and the conclusions much less pessimistic [219].

Another interesting idea pointed out by Ratra 214] is that the electromagnetic field may be directly coupled to the inflaton field. In this case the coupling is specified through a parameter $\alpha$, i.e. $e^{\alpha \varphi} F_{\alpha \beta} F^{\alpha \beta}$ where $\varphi$ is the inflaton field in Planck units. In order to get sizable large-scale magnetic fields the effective gauge coupling must be larger than one during inflation (recall that $\varphi$ is large, in Planck units, at the onset of inflation).

In 220] it has been suggested that the evolution of the Abelian gauge coupling during inflation induce the growth of the two-point function of magnetic inhomogeneities. This model is different from the one previously discussed 214. Here the dynamics of the gauge coupling is not related to the dynamics of the inflaton which is not coupled to the Abelian field strength. In particular, $r_{B}(\mathrm{Mpc})$ can be as large as $10^{-12}$. In [220] the MHD equations have been generalized to the case of evolving gauge coupling. Recently a scenario similar to [220] has been discussed in [221].

In the perspective of generating large scale magnetic fields Gasperini [222] suggested to consider the possible mixing between the photon and the graviphoton field appearing in supergravity theories (see also, in a related context 223]). The graviphoton is the massive vector component of the gravitational supermultiplet and its interaction with the photon is specified by an interaction term of the type $\lambda F_{\mu \nu} G^{\mu \nu}$ where $G_{\mu \nu}$ is the filed strength 
of the massive vector. Large-scale magnetic fields with $r_{B}(\mathrm{Mpc}) \geq 10^{-34}$ can be obtained if $\lambda \sim \mathcal{O}(1)$ and for a mass of the vector $m \sim 10^{2} \mathrm{TeV}$.

Bertolami and Mota 224 argue that if Lorentz invariance is spontaneously broken, then photons acquire naturally a coupling to the geometry which is not gauge-invariant and which is similar to the coupling considered in 213 .

Finally Davis and Dimopoulos [225] considered the possibility of phase transitions taking place during inflation. They found that sizable large-scale magnetic fields can be generated provided the phase transition occurs in the last 5 e-foldings of the inflationary stage of expansion.

\subsubsection{Abelian Higgs model}

While the coupling of electromagnetic field to the metric and to the charged fields is conformally invariant, the coupling of the charged scalar field to gravity is not. Thus, vacuum fluctuations of the charged scalar field can be amplified during inflation at super-horizon scales, leading potentially to non-trivial correlations of the electric currents and charges over cosmological distances. The fluctuations of electric currents, in turn, may induce magnetic fields through Maxwell equations at the corresponding scales. The role of the charged scalar field may be played by the Higgs boson which couples to the hypercharge field above the electroweak phase transition; the generated hypercharged field is converted into ordinary magnetic field at the temperatures of the order of electroweak scale.

No detailed computations were carried out in [213] in order to substantiate this idea. The suggestion of [213] was further developed quite recently in 226. for the standard electroweak theory with an optimistic conclusion that large scale magnetic fields can be indeed generated. In [227] a supersymmetric model was studied. In 134, previous treatments have been further scrutinized by computing, with higher accuracy, the amplification of the charged scalar field and the damping induced by the conductivity. It turns out that the resulting magnetic fields are insufficient in order to provide reasonable seeds for the dynamo amplification.

Introducing appropriately rescaled fields the action of the Abelian-Higgs model in a conformally flat FRW space-time can be written as

$$
S=\int d^{3} x d \eta\left[\eta^{\mu \nu} D_{\mu} \tilde{\phi}^{*} D_{\nu} \tilde{\phi}+\left(\frac{a^{\prime \prime}}{a}-m^{2} a^{2}\right) \tilde{\phi}^{*} \tilde{\phi}-\frac{1}{4} F_{\alpha \beta} F^{\alpha \beta}\right] .
$$


Now, since the evolution equation of the charged scalar is not conformally invariant, current density and charge density fluctuations will be induced. Then, by employing a Vlasov-Landau description similar to the one introduced in Section 5, the resulting magnetic field will be of the order of $B_{\mathrm{dec}} \sim 10^{-40} T_{\mathrm{dec}}^{2}$ which is, by far, too small. Later it has been proposed that much larger magnetic fields may be obtained in the context of the AbelianHiggs model [228] (see however [229] for a detailed criticism of this proposal).

\subsubsection{Internal dimensions}

If internal dimensions are dynamical, then Weyl invariance may be naturally broken 230]. Consider a pure electromagnetic fluctuation decoupled from the sources, representing an electromagnetic wave propagating in the $d$-dimensional external space such that $A_{\mu} \equiv A_{\mu}(\vec{x}, \eta), A_{a}=0$. In the metric given in Eq. (5.1) the evolution equation of the gauge field fluctuations can be written as

$$
\frac{1}{\sqrt{-G}} \partial_{\mu}\left(\sqrt{-G} G^{\alpha \mu} G^{\beta \nu} F_{\alpha \beta}\right)=0,
$$

where $F_{\alpha \beta}=\nabla_{[\alpha} A_{\beta]}$ is the gauge field strength and $G$ is the determinant of the $D$ dimensional metric. Notice that if $n=0$ the space-time is isotropic and, therefore, the Maxwell's equations can be reduced (by trivial rescaling) to the flat space equations. If $n \neq 0$ we have that the evolution equation of the electromagnetic fluctuations propagating in the external $d$-dimensional manifold will receive a contribution from the internal dimensions which cannot be rescaled away [233] ${ }^{26}$. In the radiation gauge $\left(A_{0}=0\right.$ and $\left.\nabla_{i} A^{i}=0\right)$ 27 the evolution the vector potentials can be written as

$$
A_{i}^{\prime \prime}+n \mathcal{F} A_{i}^{\prime}-\vec{\nabla}^{2} A_{i}=0, \quad \mathcal{F}=\frac{b^{\prime}}{b} .
$$

The vector potentials $A_{i}$ are already rescaled with respect to the (conformally flat) $d+1$ dimensional metric. In terms of the canonical normal modes of oscillations $\mathcal{A}_{i}=b^{n / 2} A_{i}$ the previous equation can be written in a simpler

\footnotetext{
${ }^{26}$ Notice that the electromagnetic field couples only to the internal dimensions through the determinant of the $D$-dimensional metric. In string theories, quite generically, the one-form fields are also coupled to the dilaton field. This case has been already analyzed in the context of string inspired cosmological scenarios and will be discussed later.

${ }^{27}$ For a discussion of gauges in curved spaces see 232 .
} 
form, namely

$$
\mathcal{A}_{i}^{\prime \prime}-V(\eta) \mathcal{A}_{i}-\vec{\nabla}^{2} \mathcal{A}_{i}=0, \quad V(\eta)=\frac{n^{2}}{4} \mathcal{F}^{2}+\frac{n}{2} \mathcal{F}^{\prime} .
$$

In order to estimate the amplification of the gauge fields induced by the evolution of the internal geometry we shall consider the background metric of Eq. (5.13) in the case of maximally symmetric subspaces $\gamma_{i j}=\delta_{i j}, \gamma_{a b}=\delta_{a b}$.

Suppose now that the background geometry evolves along three different epochs. During the first phase (taking place for $\left.]-\infty,-\eta_{1}\right]$ ) the evolution is truly multidimensional. At $\eta=-\eta_{1}$ the multidimensional dynamics is continuously matched to a radiation dominated phase turning, after decoupling, into a matter dominated regime of expansion. During the radiation and matter dominated stages the internal dimensions are fixed to their (present) constant size in order not to conflict with possible bounds arising both at the $\mathrm{BBN}$ time and during the matter-dominated epoch. The evolution of the external dimensions does not affect the amplification of the gauge fields as it can be argued from Eq. (6.36) :in the limit $n \rightarrow 0$ (i.e. conformally invariant background) Eq. (6.36) reduces to the flat space equation. A background with the features described above has been introduced in Eq. (5.14).

Defining, respectively, $b_{B B N}$ and $b_{0}$ as the size of the internal dimensions at the $\mathrm{BBN}$ time and at the present epoch, the maximal variation allowed to the internal scale factor from the BBN time can be expressed as $b_{B B N} / b_{0} \sim$ $1+\epsilon$ where $|\epsilon|<10^{-2}$ [116, 117, 118. The bounds on the variation of the internal dimensions during the matter dominated epoch are even stronger. Denoting with an over-dot the derivation with respect to the cosmic time coordinate, we have that $|\dot{b} / b|<10^{-9} H_{0}$ where $H_{0}$ is the present value of the Hubble parameter [116]. The fact that the time evolution of internal dimensions is so tightly constrained for temperatures lower of $1 \mathrm{MeV}$ does not forbid that they could have been dynamical prior to that epoch 231.

In the parameterization of Eq. (5.14) the internal dimensions grow (in conformal time) for $\lambda<0$ and they shrink ${ }^{28}$ for $\lambda>0$.

\footnotetext{
${ }^{28}$ To assume that the internal dimensions are constant during the radiation and matter dominated epoch is not strictly necessary. If the internal dimensions have a time variation during the radiation phase we must anyway impose the BBN bounds on their variation 116, 117, 118. The tiny variation allowed by BBN implies that $b(\eta)$ must be effectively constant for practical purposes.
} 
By inserting this background into Eq. (6.36) we obtain that for $\eta<-\eta_{1}$

$$
V(\eta)=\frac{n \lambda}{4 \eta^{2}}(n \lambda-2),
$$

whereas $V(\eta) \rightarrow 0$ for $\eta>-\eta_{1}$. Since $V(\eta)$ goes to zero for $\eta \rightarrow \pm \infty$ we can define, in both limits, a Fourier expansion of $\mathcal{A}_{i}$ in terms of two distinct orthonormal sets of modes. The amplification of the quantum mechanical fluctuations of the gauge fields can then be computed using the standard techniques (see, for instance, 234, 235, 236]). In 230 it has been shown that in simple models of dimensional decoupling magnetic fields can be generated with strength compatible with the bound of Eq. (6.2). In particular there is the interesting possibility that large-scale magnetic fields are produced in the case when internal dimensions also expand while the external ones also expand but at a different rate.

It should be mentioned that the interplay between gauge fields and large (or infinite) extra-dimensions is still under investigation. There is, at the moment, no definite model leading to the generation of large-scale magnetic field in a framework of infinite internal dimensions. The main reason is that it is difficult to build reasonable cosmological models with large extradimensions. More specifically, one would like to have a model where gauge fields arise naturally in the bulk. In six- dimensions interesting models can be constructed 239] where localized gauge zero modes may be naturally present (in analogy with what happens in the case of six-dimensional warped models [240, 241).

\subsubsection{String cosmological models}

Large scale magnetic fields may also be produced in the context of string cosmological models [202, 289] (see also [238] for the analysis of the amplification of magnetic fields during a phase of coherent dilaton oscillations ${ }^{29}$ ). In many respects the case of string cosmological models is related to the one where the gauge coupling is dynamical. However, in the string cosmological context, the dynamics of the gauge coupling and of the geometry are connected by the solutions of the low-energy $\beta$-functions. The basic evolution of the background and of the gauge coupling has been discussed in Section

\footnotetext{
${ }^{29}$ The possible production of (short scale) magnetic fields by parametric resonance explored in [238], has been subsequently analyzed in the standard inflationary contex [203]
} 
5. In order to achieve a large amplification of the quantum fluctuations of the gauge fields, the gauge coupling should be sufficiently small when the typical scale of the gravitational collapse hits the "potential barrier". In particular, $g_{\mathrm{ex}}\left(\omega_{\mathrm{G}}\right)<10^{-33}$ is the dynamo requirement has to be satisfied. It is difficult to produce large scale magnetic fields with reasonable amplitudes if the pre-big bang phase matches immediately to the post-big bang evolution [237]. However, phenomenologically consistent pre-big bang models lead to a sufficiently long stringy phase when the dilaton and the curvature scale are roughly constant. If this phase is included the conditions expressed by Eqs. (6.1) and (6.2) are easily satisfied. Furthermore, there are regions in the parameter space of the model where $r_{\mathrm{B}}\left(\omega_{\mathrm{G}}\right) \sim 10^{-8}$ which implies that the galactic magnetic field may be fully primordial [202, 289, i.e. no dynamo action will be required.

\subsection{Inside the Hubble radius}

The experimental evidence concerning large-scale magnetic fields suggests that magnetic fields should have similar strength over different length-scales. In this sense inflationary mechanisms seem to provide a rather natural explanation for the largeness of the correlation scale. At the same time, the typical amplitude of the obtained seeds is, in various models, rather minute.

Primordial magnetic fields can also be generated through physical mechanisms operating inside the Hubble radius at a given physical time. Particularly interesting moments in the life of the Universe are the epoch of the electroweak phase transition (EWPT) or the QCD phase transition where magnetic fields may be generated according to different physical ideas. In the following the different proposals emerged so far will be reviewed.

\subsubsection{Phase transitions}

At the time of the EWPT the typical size of the Hubble radius is of the order of $3 \mathrm{~cm}$ and the temperature is roughly $100 \mathrm{GeV}$. Before getting into the details of the possible electroweak origin of large-scale magnetic fields it is useful to present a kinematical argument based on the evolution of the correlation scale of the magnetic fields [142].

Suppose that, thanks to some mechanism, sufficiently large magnetic fields compatible with the critical density bound are generated inside the

Hubble radius at the electroweak epoch. Assuming that the typical coherence 
length of the generated magnetic fields is maximal, the present correlation scale will certainly be much larger but, unfortunately, it does not seem to be as large as the Mpc scale at the epoch of the gravitational collapse. As already mentioned, the growth of the correlation scale may be enhanced, by various processes such as inverse cascade and helical inverse cascade. For instance, if the injection spectrum generated at the electroweak epoch is Gaussian and random a simple estimate shows that the present correlation scale is of the order of $100 \mathrm{AU}$ which is already larger than what one would get only from the trivial expansion of length-scales (i.e. 1 AU) 142 . If, in a complementary perspective, the injection spectrum is strongly helical, then the typical correlation scale can even be of the order of $100 \mathrm{pc}$ but still too small than the typical scale of the gravitational collapse.

Large-scale magnetic fields can be generated at the electroweak epoch in various ways. Consider first the case when the phase transition is strongly first order.

Hogan [152 originally suggested the idea that magnetic fields can be generated during first-order phase transitions. Since during the phase transition there are gradients in the radiation temperature, similar thermoelectric source terms of MHD equations (which were discussed in the context of the Biermann mechanism) may arise. The magnetic fields, initially concentrated on the surface of the bubbles, are expelled when bubbles collide thanks to the finite value of the conductivity.

The idea that charge separation can be generated during first-order phase transitions has been exploited in 242. The suggestion is again that there are baryon number gradients at the phase boundaries leading to thermoelectric terms. In the process of bubble nucleation and collisions turbulence is then produced. In spite of the fact that the produced fields are sizable, the correlation scale, as previously pointed out, is constrained to be smaller than 100 pc.

In a first-order phase transition the phases of the complex order parameter of the nucleated bubbles are not correlated. When the bubbles undergo collisions a phase gradient arises leading to a source terms for the evolution equation of the gauge fields. Kibble and Vilenkin [243] proposed a gaugeinvariant difference between the phases of the Higgs field in the two bubbles. This idea has been investigated in the context of the Abelian-Higgs model 244, 245, 246. The collision of two spherical bubbles in the Abelian-Higgs model leads to a magnetic field which is localized in the region at the intersection of the two bubbles. The estimate of the strength of the field depends 
crucially upon the velocity of the bubble wall. The extension of this idea to the case of the standard model $S U(2) \times U(1)$ has been discussed in 247. A relevant aspect to be mentioned is that the photon field in the broken phase of the electroweak theory should be properly defined. In [248] it has been shown that the definition employed in [247] is equivalent to the one previously discussed in [249].

It has been argued by Vachaspati [250] that magnetic fields can be generated at the electroweak time even if the phase transition is of second order. The observation is that, provided the Higgs field fluctuates, electromagnetic fields may be produced since the gradients of the Higgs field appear in the definition of the photon field in terms of the hypercharge and $S U(2)$ fields. Two of the arguments proposed in 250 have been scrutinized in subsequent discussions. The first argument is related to the averaging which should be performed in order to get to the magnetic field relevant for the MHD seeds. Enqvist and Olesen [251] noticed that if line averaging is relevant the obtained magnetic field is rather strong. However [251] (see also [150]) volume averaging is the one relevant for MHD seeds.

The second point is related to the fact that the discussion of [250] was performed in terms of gauge-dependent quantities. The problem is then to give a gauge-invariant definition of the photon field in terms of the standard model fields. As already mentioned this problem has been addressed in [247] and the proposed gauge-invariant is equivalent [248] to the one proposed in 249.

In 252 a mechanism for the generation of magnetic fields at the electroweak epoch has been proposed in connection with the AMHD equations. The idea is to study the conversion of the right-electron chemical potential into hypercharge fields. In this context the baryon asymmetry is produced at some epoch prior to the electroweak phase. The obtained magnetic fields are rather strong (i.e. $|\vec{B}| \sim 10^{22} \mathrm{G}$ at the EW epoch) but over a small scale , i.e. $10^{-6} H_{\text {ew }}^{-1}$ dangerously close to the diffusivity scale.

The final point to be mentioned is that, probably, the electroweak phase transition is neither first order nor second order but it is of even higher order at least in the context of the minimal standard model [253, 254]. This conclusion has been reached using non-perturbative techniques and the relevant point, in the present context, is that for Higgs boson masses larger than $m_{W}$ the phase transition seems to disappear and it is possible to pass from the symmetric to the broken phase without hitting any first or second order phase transition. 
There have been also ideas concerniing the a possible generation of magnetic fields at the time of the QCD phase transition occurring roughly at $T \sim 140 \mathrm{MeV}$, i.e. at the moment when free quarks combine to form colorless hadrons. The mechanism here is always related to the idea of Biermann with thermoelectric currents developed at the QCD time.Since the strange quark is heavier than the up and down quarks there may be the possibility that the quarks develop a net positive charge which is compensated by the electric charge in the leptonic sector. Again, invoking the dynamics of a first-order phase transition, it is argued that the shocks affect leptons and quarks in a different way so that electric currents are developed as the bubble wall moves in the quark-gluon plasma. In 255] the magnetic field has been estimated to be $|\vec{B}| \sim \mathrm{G}$ at the time of the QCD phase transition and with typical scale of the order of the meter at the same epoch.

In [256, 257] it has been pointed out that, probably, the magnetic fields generated at the time of QCD phase transition may be much stronger than the ones estimated in [255]. The authors of [256, 257] argue that strong magnetic fields may be generated when the broken and symmetric phase of the theory coexist. The magnetic fields generated at the boundaries between quark and hadron phases can be, according to the authors, as large as $10^{6} \mathrm{G}$ over scales of the order of the meter at the time of the QCD phase transition.

Recently, in a series of papers, Boyanovsky, de Vega and Simionato [258, 259, 260] studied the generation of large scale magnetic fields during a phase transition taking place in the radiation dominated epoch. The setting is a theory of $N$ charged scalar fields coupled to an Abelian gauge field that undergoes a phase transition at a critical temperature much larger than the electroweak scale. Using non-equilibrium field theory techniques the authors argue that during the scaling regime (when the back-reaction effects are dominant) large scale magnetogenesis is possible. The claim is that the minimal dynamo requirement of Eq. (6.1) is achievable at the electroweak scale. Furthermore, much larger magnetic fields can be expected if the scaling regime can be extended below the QCD scale.

\subsection{Mixed mechanisms}

From the above discussion it is apparent that the mechanisms producing magnetic fields at some moment in the life of the Universe (and inside the Hubble radius) have to justify the large correlation scale of the magnetic seeds required by subsequent MHD considerations. The possibility of turbulent 
behaviour in the early Universe, even though physically justified, seems to be difficult to quantify even if rather reasonable quantitative estimates appeared so far. On the other hand, inflationary scenarios can lead to magnetic seeds with large correlation scale.

A useful point to be stressed is that there may exist "mixed" mechanisms. Consider the following example which has been proposed in [171] and 172. Suppose that during the inflationary phase a stochastic background of gauge fields is produced thanks to the breaking of conformal invariance. The spectral amplitude of the generated field will be constrained by the critical density bound and by all the other dynamical considerations related to the finite values of the diffusion scales.

The generated magnetic field spectrum will be distributed over a large interval of frequencies. In particular, the energy spectrum of the magnetic field will be non negligible at some intermediate scale, like, for instance, the electroweak scale, i.e. the physical frequency $\omega_{\mathrm{ew}} \sim H_{\mathrm{ew}}$. In other words, if magnetic fields are generated thanks to some inflationary mechanism, the amplified magnetic inhomogeneities will be reentering at different epochs during the radiation dominated phase and the scales which left the horizon later during inflation will reenter earlier.

Given the background of abelian gauge fields, the dynamics of the electroweak epoch may add interesting physical features. For instance the magnetic field of inflationary origin will be, as discussed, topologicaly trivial: its magnetic gyrotropy will vanish. However, during the EW epoch, thanks to a pseudoscalar vertex due either to a pseudo goldstone boson or to the chemical pootential, the topologically trivial hypermagnetic background may be turned into a topologically non-trivial background and large magnetic helicity may be generated.

\section{Effects of primordial magnetic fields}

In the context of various mechanisms, the magnetic fields generated at large at the scale of the protogalactic collapse may be rather small. In this Section the possible effects of magnetic present over much smaller length-scales will be analyzed. Two important scales are the Hubble radius at the electroweak epoch and at the big-bang nucleosynthesis epoch. 


\section{$7.1 \quad$ Electroweak epoch}

Hypermagnetic fields present for temperatures $T \geq 100 \mathrm{GeV}$ have a twofold effect:

- they may affect the phase diagram of the electroweak theory;

- they may offer a mechanism to seed the baryon asymmetry of the Universe $(\mathrm{BAU})$.

\subsubsection{EW phase diagram}

The physical picture of the possible effects of magnetic fields on the electroweak phase diagram is exactly the same as the macroscopic description of superconductors in the presence of an external magnetic field. The normalsuperconducting phase transition, being of second order in the absence of magnetic fields, becomes of first order if a magnetic field is present. The reason for this is the Meissner effect, i.e. the fact that the magnetic field cannot propagate inside a superconducting cavity, and, therefore, creates an extra pressure acting on the normal-superconducting boundary. Our consideration below explores this simple picture.

Consider the plain domain wall that separates broken and symmetric phase at some temperature $T$, in the presence of a uniform hypercharge magnetic field $Y_{j}$. Far from the domain wall, in the symmetric phase, the non-Abelian $\mathrm{SU}(2)$ field strength $\left(W_{j}^{3}\right)$ is equal to zero, because of a nonperturbative mass gap generation. Inside the broken phase, the massive $Z_{j}$ combination of $Y_{j}$ and $W_{j}^{3}$,

$$
Z_{j}=\cos \theta_{W} W_{j}^{3}-\sin \theta_{W} Y_{j}
$$

must be equal to zero, while the massless combination, corresponding to photon $A_{j}^{e m}$, survives. The matching of the fields on the boundary gives $A_{j}^{e m}=\mathcal{Y}_{j} \cos \theta_{W}$. Thus, an extra pressure $\frac{1}{2}\left|\overrightarrow{\mathcal{H}}_{Y}\right|^{2} \sin ^{2} \theta_{W}$ acts on the domain wall from the symmetric side. At the critical temperature it must be compensated by the vacuum pressure of the scalar field. If we neglect loop corrections associated with the presence of magnetic fields, then the condition that determines the critical temperature is:

$$
\frac{1}{2}\left|\vec{H}_{Y}\right|^{2} \sin ^{2} \theta_{W}=V\left(0, T_{c}\right)-V\left(\varphi_{\min }, T_{c}\right)
$$


where $V(\varphi, T)$ is the effective potential in the absence of magnetic field, $\varphi_{\min }$ is the location of the minimum of the potential at temperature $T$.

The above consideration was dealing with the uniform magnetic fields. Clearly, it remains valid when the typical distance scale of inhomogeneities of the magnetic field are larger than the typical bubble size. This is the case for bubbles smaller than the magnetic diffusion scale, and, in particular, at the onset of the bubble nucleation. Thus, the estimate of the critical temperature coming from (17.2) is applicable. For bubbles larger than the diffusivity scale, the presence of a stochastic magnetic field will considerably modify their evolution. In particular, the spherical form of the bubbles is very likely to be spoiled.

These considerations were presented in [163]. Later [261] perturbative estimates were performed in order to corroborate the proposed picture. In [262] a full non-perturbative analysis of the phhase diagram of the electroweak theory in the presence of an hyermagnetic background has been performed. As previously discussed [253, 254] for values of the Higggs boson mass larger than the $\mathrm{W}$ boson mass the electroweak phase diagram seems to exhibit a cross-over regime. The inclusion of a constant hypermagnetic background with typical strength $\left|\vec{H}_{Y}\right| / T^{2} \leq 0.3$ does modify the electroweak phase diagram but does not seem to make the phase transition strongly first order for $m_{H} \geq m_{W}$ as expected from perturbative considerations. Furthermore for $\left|\vec{H}_{Y}\right| / T^{2}>0.3$ (but still compatible with the critical density bound) a new (inhomogeneous) phase has been observed. The analysis performed in 262 included a net hypermagnetic flux. It would be interesting to repeat the same calculation in the presence of a non-vanishing hypermagnetic helicity (or gyrotropy).

\subsubsection{Baryon asymmetry}

Depending upon the topology of the flux lines, hypermagnetic fields can have two distinct effects:

- topologically non-trivial configurations of the hypermagnetic flux lines lead to the formation of hypermagnetic knots whose decay might seed the Baryon Asymmetry of the Universe (BAU);

- even if the electrowak plasma has vanishing hypermagnetic gyrotropy, i.e. $\left\langle\vec{H}_{Y} \cdot \vec{\nabla} \times \vec{H}_{Y}\right\rangle=0$, matter-antimatter fluctuations may be generated. 
Consider first the situation where the electroweak plasma contains, for $T>$ $T_{c}$ a network of hypermagnetic knots of the type of the ones described in Eqs. (5.113) - (5.116). In Section 5 these configurations have been named hypermagnetic knots (HK) and they are Chern-Simons condensates carrying a non-vanishing (averaged) hypermagnetic helicity. As discussed, HK can be dynamically generated from a background of hypermagnetic fields with trivial topology provided a (time-dependent) pseudo-scalar is present in the plasma (see, for instance, Eq. (5.120) ).

In order to seed the BAU a network of HK should be present at high temperatures. In fact for temperatures larger than $T_{c}$ the fermionic number is stored both in HK and in real fermions. For $T<T_{c}$, the HK should release real fermions since the ordinary magnetic fields (present after EW symmetry breaking) do not carry fermionic number. If the EWPT is strongly first order the decay of the HK can offer some seeds for the BAU generation. This last condition can be met in the minimal supersymmetric standard model (MSSM).

The integration of the $U(1)_{Y}$ anomaly equation gives the CS number density carried by the HK which is in turn related to the density of baryonic number $n_{B}$ for the case of $n_{f}$ fermionic generations. In fact, using Eqs. (5.109) - (5.111) and after some algebra [163, 172] it can be shown that

$$
\frac{n_{B}}{s}\left(t_{c}\right)=\frac{\alpha^{\prime}}{2 \pi \sigma_{c}} \frac{n_{f}}{s} \frac{\left\langle\vec{H}_{Y} \cdot \vec{\nabla} \times \vec{H}_{Y}\right\rangle}{\Gamma+\Gamma_{H}} \frac{M_{0} \Gamma}{T_{c}^{2}}, \quad \alpha^{\prime}=\frac{g^{\prime 2}}{4 \pi}
$$

( $g^{\prime}$ is the $U(1)_{Y}$ coupling and $s=(2 / 45) \pi^{2} g_{*} T^{3}$ is the entropy density; $g_{*}$, at $T_{c}$, is 106.75 in the MSM; $\left.M_{0}=M_{P} / 1.66 \sqrt{g_{*}}\right)$. In Eq. (17.3) $\Gamma$ is the perturbative rate of the right electron chirality flip processes (i.e. scattering of right electrons with the Higgs and gauge bosons and with the top quarks because of their large Yukawa coupling) which are the slowest reactions in the plasma and

$$
\Gamma_{H}=\frac{783}{22} \frac{\alpha^{\prime 2}}{\sigma_{c} \pi^{2}} \frac{\left|\vec{H}_{Y}\right|^{2}}{T_{c}^{2}}
$$

is the rate of right electron dilution induced by the presence of a hypermagnetic field. In the MSM we have that $\Gamma<\Gamma_{H}$ whereas in the MSSM $\Gamma$ can naturally be larger than $\Gamma_{H}$. Unfortunately, in the MSM a hypermagnetic field can modify the phase diagram of the phase transition but cannot make the phase transition strongly first order for large masses of the Higgs boson. Therefore, we will concentrate on the case $\Gamma>\Gamma_{H}$ and we will show that in 
the opposite limit the BAU will be anyway small even if some (presently unknown) mechanism would make the EWPT strongly first order in the MSM.

It is interesting to notice that, in this scenario, the value of the BAU is determined by various particle physics parameters but also by the ratio of the hypermagnetic energy density over the energy density sitting in radiation during the electroweak epoch.

Consider now the complementary situation where the electroweak plasma, for $T>T_{c}$, is filled with topologically trivial hypermagnetic fields. In this case, fluctuations in the baryon to entropy ratio will be induced since, in any case $\left\langle\left(\vec{H}_{Y} \cdot \vec{\nabla} \times \vec{H}_{Y}\right)^{2}\right\rangle \neq 0$. These fluctuations are of isocurvature type and can be related to the spectrum of hypermagnetic fields at the EWPT. Defining as

$$
\Delta\left(r, t_{c}\right)=\sqrt{\left\langle\delta\left(\frac{n_{B}}{s}\right)\left(\vec{x}, t_{c}\right) \delta\left(\frac{n_{B}}{s}\right)\left(\vec{x}+\vec{r}, t_{c}\right)\right\rangle}
$$

the fluctuations in the ratio of baryon number density $n_{B}$ to the entropy density $s$ at $t=t_{c}$ 164, the value of $\Delta\left(r, t_{c}\right)$ can be related to the hypermagnetic spectrum which is determined in terms of its amplitude $\xi$ and its slope $\epsilon$. A physically realistic situation corresponds to the case in which the Green's functions of the magnetic hypercharge fields decay at large distance (i. e. $\epsilon>0)$ and this would imply either "blue" $(\epsilon \geq 0)$ or "violet" $(\epsilon \gg 1)$ energy spectra. The fluctuations of the baryon to entropy ratio generated at the electroweak epoch may survive until the BBN epoch [164]. The possibility of survival of these fluctuations is related to their typical scale which must exceed the neutron diffusion scale appropriately blue-shifted at the electroweak epoch. The implications of these fluctuations will be discussed in a moment.

\subsection{Big-bang nucleosynthesis epoch}

Large scale magnetic fields possibly present at the BBN epoch can have an impact on the light nuclei formation. By reversing the argument, the success of BBN can be used in order to bound the magnetic energy density possibly present at the time of formation of light nuclei. Magnetic fields, at the BBN epoch may have four distinct effects:

- they can enhance the rate of the Universe expansion at the corresponding epoch in a way proportional to $\rho_{\mathrm{B}}$; 
- they can affect the reaction rate in a way proportional to $\alpha_{\mathrm{em}} \rho_{\mathrm{B}}$;

- they can affect the phase space of the electrons;

- they can induce, prior to the formation of the light nuclei, matterantimatter fluctuations.

While the first three effects are direct, the fourth effect is mainly caused by hypermagnetic fields present even before BBN.

The idea that magnetic fields may increase the Universe expansion and, consequently, affect directly the abundance of ${ }^{4} \mathrm{He}$ (which is directly sensitive to the expansion rate) has been pointed out long ago by Greenstein 264] and by Matese and O'Connel [265]. From a qualitative point of view the effects over the expansion should be leading in comparison with the effects over the rate of interactions (which are suppressed by $\alpha_{\mathrm{em}}$ ).

Thanks to detailed numerical simulations performed indipendently by different groups (i.e. Cheng et al. [266, 267]; Kernan, Starkman and Vachaspati 268, 269]; Grasso and Rubinstein [270, 271]), the common opinion is that the Universe expansion is the leading effect even if this conclusion has been reached only recently and not without some disagreements on the details of the calculations (see, for instance, [269]).

In order to prevent the Universe from expanding too fast at the BBN epoch $\rho_{B}<0.27 \rho_{\nu}$ where $\rho_{\nu}$ is the energy density contributed by the standard three light neutrinos with masses much smaller than the MeV. The $\mathrm{BBN}$ bound is physically relevant in many situations since it is a bit more constraining than the critical density bound.

The BBN bounds discussed so far are derived assuming a stochastic magnetic field at the BBN epoch. This means that the isotropy of the geometry is not affected. However, it is not unreasonable to think of the possibility that a magnetic field with a preferred direction may break the isotropy of the background. Thus bounds on the isotropy of the expansion at the BBN epoch may be turned into bounds on the shear induced by the presence of a magnetic field (see, for instance, [272, 273] and [274, 275]).

Let us now come to the last point. The matter-antimatter fluctuations created at the electroweak epoch thanks to the presence of hypermagnetic fields may survive down to the epoch of BBN. Since the fluctuations in the baryon to entropy ratio are, in general, not positive definite, they will induce fluctuations in the baryon to photon ratio, at the BBN epoch. The possible effect of matter-antimatter fluctuations on BBN depends on the typical scale 


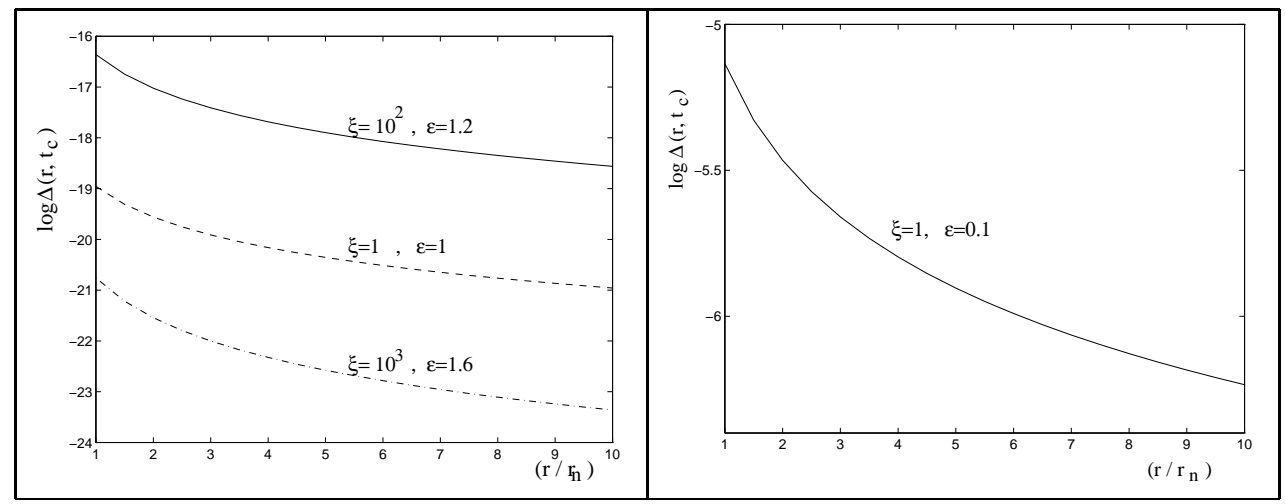

Figure 8: The value of the baryon number fluctuations for different parameters of the hypermagnetic background $\xi$ and $\epsilon$ is reported.

of of the baryon to entropy ratio at the electroweak epoch. Recalling that for $T \sim T_{c} \sim 100 \mathrm{GeV}$ the size of the electrowek horizon is approximately $3 \mathrm{~cm}$, fluctuations whose scale is well inside the EW horizon at $T_{c}$ have dissipated by the BBN time through (anti)neutron diffusion. The neutron diffusion scale at $T_{c}$ is

$$
r_{\mathrm{n}}\left(T_{c}\right)=0.3 \mathrm{~cm}
$$

The neutron diffusion scale at $T=1 \mathrm{keV}$ is $10^{5} \mathrm{~m}$, while, today it is $10^{-5}$ pc, i.e. of the order of the astronomical unit. Matter-antimatter fluctuations smaller than $10^{5} \mathrm{~m}$ annihilate before neutrino decoupling and have no effect on BBN. Two possibilities can then be envisaged. We could require that the matter-antimatter fluctuations (for scales $r \geq r_{\mathrm{n}}$ ) are small. This will then imply a bound, in the $(\xi, \epsilon)$ plane on the strength of the hypermagnetic background. In Fig. 8 some typical baryon number fluctuations have been reported for different choices of the parameters $\xi$ and $\epsilon$. In Fig. 9] such an exclusion plot is reported with the full line. With the dashed line the bound implied by the increase in the expansion rate (i.e. $\rho_{H}<0.2 \rho_{\nu}$ ) is reported. Finally with the dot-dashed line the critical density bound is illustrated for the same hypermagnetic background. The second possibility is to study the effects of large matter-matter domains. These studies led to a slightly different scenario of BBN [276], namely BBN with matter-antimatter regions. This analysis has been performed in a series of papers by Rehm and Jedamzik [277, 282 and by Kurki-Suonio and Sihvola [278, 279] (see also [280, 281]). The idea is to discuss BBN in the presence of spherically symmetric regions 


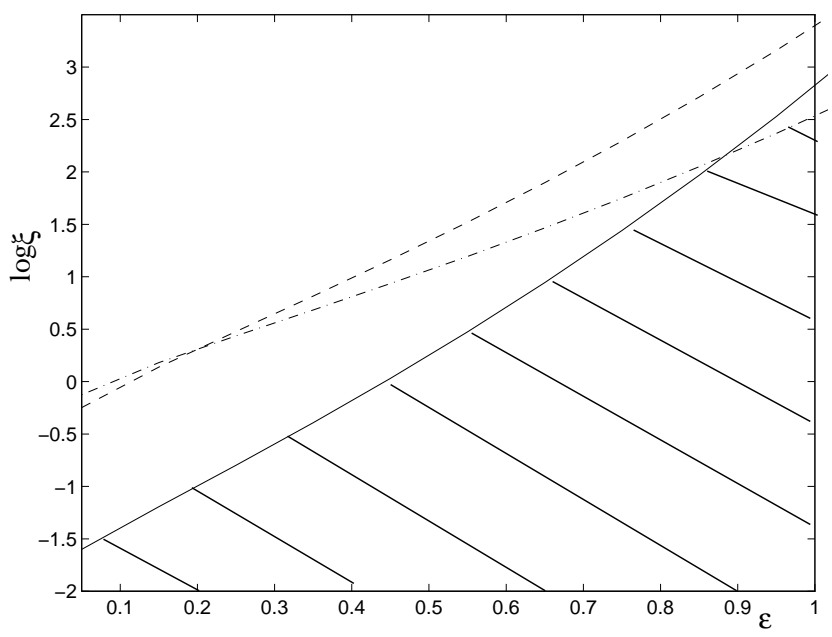

Figure 9: The parameter space of the hypermagnetic background in the case $\Delta\left(r, t_{c}\right)<n_{B} / s$ for $r>r_{\mathrm{n}}$ (full line).

of anti-matter characterized by their radius $r_{\mathrm{A}}$ and by the parameter $R$, i.e. the matter/antimatter ratio. Furthermore, in this scenario the net baryonto-photon ratio, $\bar{\eta}$, is positive definite and non zero. Antimatter domains larger than $10^{5} \mathrm{~m}$ at $1 \mathrm{keV}$ may survive until BBN and their dissipation has been analyzed in detail [279]. Antimatter domains in the range

$$
10^{5} \mathrm{~m} \leq r_{\mathrm{A}} \leq 10^{7} \mathrm{~m}
$$

at $1 \mathrm{keV}$ annihilate before BBN for temperatures between $70 \mathrm{keV}$ and $1 \mathrm{MeV}$. Since the antineutrons annihilate on neutrons, the neutron to proton ratio gets smaller. As a consequence, the ${ }^{4} \mathrm{He}$ abundance gets reduced if compared to the standard BBN scenario. The maximal scale of matter-antimatter fluctuations is determined by the constraints following from possible distortions of the CMB spectrum. The largest scale is of the order of $100 \mathrm{pc}$ (today), corresponding to $10^{12} \mathrm{~m}$ at $1 \mathrm{keV}$. Suppose that matter-antimatter regions are present in the range of Eq. (7.7). Then the abundance of ${ }^{4} \mathrm{He}$ get reduced. The yield of ${ }^{4} \mathrm{He}$ are reported as a function of $R$, the matterantimatter ratio and $r_{\mathrm{A}}$. Now, we do know that by adding extra-relativistic species the ${ }^{4} \mathrm{He}$ can be increased since the Universe expansion gets larger. Then the conclusion is that BBN with matter-antimatter domains allows for a larger number of extra-relativistic species if compared to the standard BBN scenario. This observation may have implications for the upper bounds on 


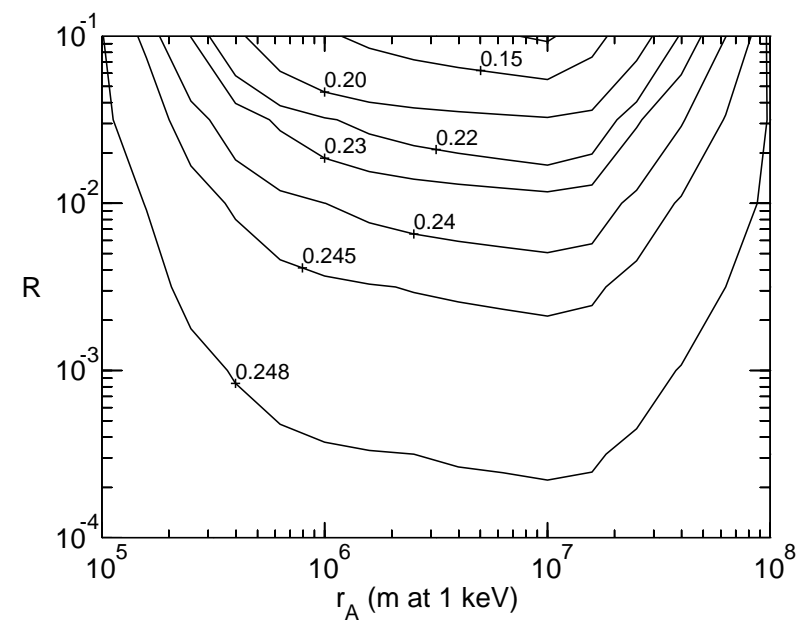

Figure 10: From [283] the ${ }^{4} \mathrm{He}$ yield is illustrated in the $\left(R, r_{\mathrm{A}}\right)$ plane for $\bar{\eta}=6 \times 10^{-10}$. As the matter/antimatter ratio decreases, we recover the standard ${ }^{4} \mathrm{He}$ yield.

the stochastic GW backgrounds of cosmological origin [283] since the extrarelativistic species present at the $\mathrm{BBN}$ epoch can indeed be interpreted as relic gravitons.

\section{CMB physics an magnetic fields}

The vacuum fluctuations of the gauge fields present during the inflationary stage of expansion may be amplified if conformal invariance is broken. It is then plausible that magnetic inhomogeneities are amplified not only at the scale of protogalactic collapse but also at larger length-scales. agnetic fields can be generated over all physical scales compatible Large-scale magnetic fields may then have various interesting implications on the physics of the $\mathrm{CMB}$ and of its anisotropies. The following possible effects have been discussed through the years:

- distortion of the Planckian spectrum;

- shift of the polarization plane (provided the CMB is linearly polarized);

- shift in the position of the first Doppler peak; 
- generic increase of the amount of the (primary) anisotropy.

On top of these effects, magnetic fields can also modify the evolution of the tensor fluctuations of the geometry for typical length scales much smaller than the ones probed by CMB anisotropy experiments.

The possible distortions of the Planckian spectrum of CMB are usually discussed in terms of a chemical potential which is bounded, by experimental data, to me $|\mu|<9 \times 10^{-5}$. Magnetic field dissipation at high red-shift implies the presence of a chemical potential. Hence bounds on the distorsion of the Planckian spectrum of the CMB can be turned into bounds on the magnetic field strength at various scales. In particular [287] the obtained bound are such that $B<3 \times 10^{-8} \mathrm{G}$ for comoving coherence lengths between $0.4 \mathrm{kpc}$ and $500 \mathrm{kpc}$.

Large scale magnetic fields can also afffect the poosition of the Doppler peak. In 288 this analysis has been performed in a non-relativistic approximation where the scalar perturbations of the geometry obey linearized Newtonian equations of motion. It has been found that, in this approximation, the effect of the presence of the magnetic fields is an effective renormalization of the speed of sound of the baryons.

\subsection{Large-scale magnetic fields and CMB anisotropies}

Large scale magnetic fields present prior to the recombination epoch may act as a source term in the evolution equation of the cosmological perturbations. If magnetic fields are created over large length scales (possibly even larger than the Mpc) it is rather plausible that they may be present after matter-radiation equality (but before decoupling) when primordial fluctuations are imprinted on the CMB anisotropies. In fact, the relevant modes determining the large scale temperature anisotropies are the ones that are outside the horizon for $\eta_{\mathrm{eq}}<\eta<\eta_{\mathrm{dec}}$ where $\eta_{\mathrm{eq}}$ and $\eta_{\mathrm{dec}}$ are, respectively, the equality and the decoupling times. After equality there are two complementary approaches which can be used in order to address phenomenological implications of large scale magnetic fields:

- large scale magnetic fields are completely homogeneous outside the horizon after equality;

- large scale magnetic fields are inhomogeneous after equality. 
The idea that homogeneous magnetic fields may affect the CMB anisotropies was originally pointed out by Zeldovich 284, 285. and further scrutinized by Grishchuk, Doroshkevich and Novikov [286]. Since this idea implies that large-scale magnetic fields are weakly gravitating the details of the discussion (and of the possible generalization of this idea) will be postponed to the following Section.

The suggestion that inhomogeneous magnetic fields created during inflation may affect the CMB anisotropies was originally proposed in [289]. In 289 it was noticed that large scale magnetic fields produced during inflation may also lead to large fluctuations for modes which are outside the horizon after equality. In 289] it was noticed that either the produced magnetic fields may be used to seed directly the CMB anisotropies, or, in a complementary persepective, the CMB data can be used to put constraints on the production mechanism.

If large-scale magnetic fields are inhomogeneous, their energy momentum tensor will have, in general terms, scalar, vector and tensor modes

$$
\delta \mathcal{T}_{\mu \nu}=\delta \mathcal{T}_{\mu \nu}^{(S)}+\delta \mathcal{T}_{\mu \nu}^{(V)}+\delta \mathcal{T}_{\mu \nu}^{(T)},
$$

which are decoupled and which act as source terms for the scalar, vector and tensor modes of the geometry

$$
\delta g_{\mu \nu}=\delta g_{\mu \nu}^{(S)}+\delta g_{\mu \nu}^{(V)}+\delta g_{\mu \nu}^{(T)}
$$

whose gauge-invariant description is well explored [199, 200] (see also 201]). The effect of the scalar component of large scale magnetic fields should be responsible, according to the suggestion of [289], of the large scale temperature anisotropies. The direct seeding of large-scale temperature anisotropies seems unlikely. In fact, the spectrum of large scale gauge fields leading to plausible values of the magnetic energy density over the scale of protogalactic collapse is typically smaller than the value required by experimental data. However, even if the primordial spectrum of magnetic fields would be tailored in an appropriate way, the initial conditions for the plasma evolution compatible with the presence of a sizable (but undercritical) magnetic field are of isocurvature type [290].

In order to illustrate this point consider the evolution equations for the gauge-invariant system of scalar perturbations of the geometry expressed in terms of the two Bardeen potential $\Phi$ and $\Psi$ corresponding, in the Newtonian gauge, to the fluctuations of the temporal and spatial component of the 
metric [200, 201]. The linearization of the $(00),(0 i)$ and $(i, j)$ components of the Einstein equations leads, respectively, to:

$$
\begin{aligned}
& \nabla^{2} \Psi-3 \mathcal{H}\left(\mathcal{H} \Phi+\Psi^{\prime}\right)=\frac{a^{2}}{2 M_{\mathrm{P}}^{2}} \rho \delta+\frac{a^{2}}{2 M_{\mathrm{P}}^{2}} \delta \mathcal{T}_{0}^{0}, \\
& \partial_{i}\left[\mathcal{H} \Phi+\Psi^{\prime}\right]=\frac{a^{2}}{2 M_{\mathrm{P}}^{2}}(1+w) \rho \partial_{i} u+\frac{a^{2}}{2 M_{\mathrm{P}}^{2}} \delta \mathcal{T}_{i}^{0}, \\
& \left\{\Psi^{\prime \prime}+\mathcal{H}\left(\Phi^{\prime}+2 \Psi^{\prime}\right)+\left(\mathcal{H}^{2}+2 \mathcal{H}^{\prime}\right) \Phi+\frac{1}{2} \nabla^{2}(\Phi-\Psi)\right\} \delta_{i}^{j} \\
& -\frac{1}{2} \partial_{i} \partial^{j}(\Phi-\Psi)=-\frac{a^{2}}{2 M_{\mathrm{P}}^{2}} \delta \mathcal{T}_{i}^{j}+w \frac{a^{2}}{2 M_{\mathrm{P}}} \rho \delta,
\end{aligned}
$$

where $\delta=\delta \rho / \rho$ and $w=p / \rho$ is the usual barotropic index, and

$$
\begin{aligned}
\delta \mathcal{T}_{0}^{0} & =\frac{1}{8 \pi a^{4}}|\overrightarrow{\mathcal{B}}(\vec{x})|^{2} \\
\delta \mathcal{T}_{i}^{j} & =\frac{1}{4 \pi a^{4}}\left[-\mathcal{B}_{i} \mathcal{B}^{j}+\frac{1}{2}|\overrightarrow{\mathcal{B}}(\vec{x})|^{2} \delta_{i}^{j}\right], \\
\delta \mathcal{T}_{i}^{0} & =\frac{\overrightarrow{\mathcal{B}} \times \vec{\nabla} \times \overrightarrow{\mathcal{B}}}{16 \pi^{2} a^{4} \sigma} .
\end{aligned}
$$

are the fluctuations of the energy-momentum tensor of the magnetic field. Eqs. (9.10) - (8.5) have to be supplemented by the perturbation of the covariant conservation equations for the fluid sources:

$$
\begin{aligned}
& \delta^{\prime}-(1+w) \nabla^{2} u-3 \Psi^{\prime}(1+w)=0, \\
& u^{\prime}+\mathcal{H}(1-3 w) u-\frac{w}{w+1} \delta-\Phi=0 .
\end{aligned}
$$

If the magnetic field is force-free, i.e. $\overrightarrow{\mathcal{B}} \times \vec{\nabla} \times \overrightarrow{\mathcal{B}}$ the above system simplifies. Notice that Eq. (8.8) has been already written in the case where the Lorentz force is absent. However, the forcing term appears, in the resistive MHD approximation, in Eq. (8.6). The term $\overrightarrow{\mathcal{B}} \times \vec{\nabla} \times \overrightarrow{\mathcal{B}}$ is suppressed by the conductivity, however, it can be also set exactly to zero. In this case, vector identities imply

$$
(\overrightarrow{\mathcal{B}} \times \vec{\nabla} \times \overrightarrow{\mathcal{B}})_{i}=\mathcal{B}_{m} \partial_{i} \mathcal{B}^{m}-\mathcal{B}_{m} \partial^{m} \mathcal{B}_{i}=0,
$$

i.e. $\mathcal{B}_{m} \partial_{i} \mathcal{B}^{m}=\mathcal{B}_{m} \partial^{m} \mathcal{B}_{i}$. With this identity in mind the system of Eqs. (8.3) - 8.5) and (8.7) - 8.8) can be written, in Fourier space, as

$$
k^{2} \Psi_{k}+3 \mathcal{H}\left(\mathcal{H} \Phi_{k}+\Psi_{k}^{\prime}\right)=-\frac{a^{2}}{2 M_{\mathrm{P}}^{2}}-\frac{\mathcal{B}_{0}^{2}(k)}{8 \pi a^{2} M_{\mathrm{P}}^{2}},
$$




$$
\begin{aligned}
& \Psi_{k}^{\prime \prime}+\mathcal{H}\left(\Phi_{k}^{\prime}+2 \Psi_{k}^{\prime}\right)+\left(2 \mathcal{H}^{\prime}+\mathcal{H}^{2}\right) \Psi \\
& -\frac{k^{2}}{3}\left(\Phi_{k}-\Psi_{k}\right)=\frac{a^{2}}{2 M_{\mathrm{P}}^{2}} w \rho \delta_{k}-\frac{\mathcal{B}_{0}^{2}(k)}{48 \pi a^{2} M_{\mathrm{P}}^{2}}, \\
& k^{2}\left(\Phi_{k}-\Psi_{k}\right)=\frac{\mathcal{B}_{0}^{2}(k)}{16 \pi a^{2} M_{\mathrm{P}}^{2}} \mathcal{B}_{0}^{2}(k), \\
& \delta_{k}^{\prime}+k^{2}(1+w) u_{k}-3 \Psi_{k}^{\prime}(w+1)=0, \\
& u_{k}^{\prime}+\mathcal{H}(1-3 w) u_{k}-\frac{w}{w+1} \delta_{k}-\Phi_{k}=0,
\end{aligned}
$$

where

$$
\mathcal{B}_{0}^{2}(k)=\frac{1}{(2 \pi)^{3}} \int d^{3} p \overrightarrow{\mathcal{B}}(\vec{p}) \cdot \overrightarrow{\mathcal{B}}(\vec{k}-\vec{p}) .
$$

Combining Eqs. (8.10) (8.11) with Eq. (8.12) the following decoupled equation can be obtained

$$
\Psi_{k}^{\prime \prime}+3 \mathcal{H}(1+w) \Psi_{k}^{\prime}+k^{2} w \Psi_{k}=\frac{\mathcal{B}_{0}^{2}(k)}{8 \pi a^{2} M_{\mathrm{P}}^{2}}\left[\frac{\mathcal{H}^{2}(1-3 w)-2 \mathcal{H}^{\prime}}{2 k^{2}}-w\right]
$$

Consider, for simplicity, the dark-matter radiation fluid after equality and in the presence of a force-free magnetic field. In this case Eq. (8.16) can be immediately integrated:

$$
\begin{aligned}
& \Psi_{k}(\eta)=\Psi_{0}(k)-\frac{\Psi_{1}(k)}{5}\left(\frac{\eta_{\mathrm{eq}}}{\eta}\right)^{5}-\frac{\mathcal{B}_{0}^{2}(k)}{8 \pi k^{2} M_{\mathrm{P}}^{2}}\left(\frac{\eta_{\mathrm{eq}}}{\eta}\right)^{4}, \\
& \Phi_{k}(\eta)=\Psi_{0}(k)-\frac{\Psi_{1}(k)}{5}\left(\frac{\eta_{\mathrm{eq}}}{\eta}\right)^{5}-\frac{\mathcal{B}_{0}^{2}(k)}{16 \pi k^{2} M_{\mathrm{P}}^{2}}\left(\frac{\eta_{\mathrm{eq}}}{\eta}\right)^{4} .
\end{aligned}
$$

The solutions given in Eq. (8.17) determine the source of the density and velocity fluctuations in the plasma, i.e.

$$
\begin{aligned}
& \delta_{\mathrm{r}}^{\prime}-\frac{4}{3} \nabla^{2} u_{\mathrm{r}}-4 \Psi^{\prime}=0, \\
& u_{\mathrm{r}}^{\prime}-\frac{1}{4} \delta_{\mathrm{r}}-\Phi=0 \\
& \delta_{\mathrm{m}}^{\prime}-\nabla^{2} u_{\mathrm{m}}-3 \Psi^{\prime}=0 \\
& u_{\mathrm{m}}^{\prime}+\mathcal{H} u_{\mathrm{m}}-\Phi=0,
\end{aligned}
$$

The solution for the velocity fields and density contrasts can be easily obtained by integrating Eqs. (8.18)-(8.21) with the source terms determined 
by Eq. (8.17). The purpose is not to integrate here this system (see for instance [291] for the standard case without magnetic fields). Rather it is important to notice that there are two qualitatively different situation. The first situation is the one where,

$$
\left|\Psi_{0}(k)\right| \ll \frac{\mathcal{B}_{0}^{2}(k)}{8 \pi k^{2} M_{\mathrm{P}}^{2}}\left(\frac{\eta_{\mathrm{eq}}}{\eta}\right)^{4}
$$

namely the case where the constant mode of the Bardeen potential is negligible if compared to the contribution of the magnetic field. The derivative of the Bardeen potential will be, however, non vanishing. In this case the CMB anisotropies are said to be seeded by isocurvature initial conditions for the fluid of radiation and dark-matter present after equality. In the opposite case, nemely

$$
\left|\Psi_{0}(k)\right| \gg \frac{\mathcal{B}_{0}^{2}(k)}{8 \pi k^{2} M_{\mathrm{P}}^{2}}\left(\frac{\eta_{\mathrm{eq}}}{\eta}\right)^{4},
$$

the constant mode of the Bardeen potential is provided, for instance, by inflation and the magnetic field represent a further parameter to be taken into account in the analysis of CMB anisotropies. Assuming, roughly, that $\left|\Psi_{0}(k)\right| \sim 10^{-6}$, Eq. (8.23) implies that for the typical scale of the horizon at decoupling the critical fraction of magnetic energy density should be smaller than $10^{-3}$. In the case of Eq. (8.23) the leading order relations among the different hydrodynamical fluctuations are well known and can be obtained from Eqs. (8.18) - (8.21) if the magnetic field is approximately negligible, i.e.

$$
k u_{\mathrm{r}} \simeq k u_{\mathrm{m}}, \quad \delta_{\mathrm{r}} \simeq(4 / 3) \delta_{\mathrm{m}} .
$$

This result has a simple physical interpretation, and implies the adiabaticity of the fluid perturbations. The entropy per matter particle is indeed proportional to $S=T^{3} / n_{\mathrm{m}}$, where $n_{\mathrm{m}}$ is the number density of matter particles and $T$ is the radiation temperature. The associated entropy fluctuation, $\delta S$, satisfies

$$
\frac{\delta S}{S}=\frac{3}{4} \delta_{\mathrm{r}}-\delta_{\mathrm{m}}
$$

where we used the fact that $\rho_{\mathrm{r}} \sim T^{4}$ and that $\rho_{\mathrm{m}}=m n_{\mathrm{m}}$, where $m$ is the typical mass of the particles in the matter fluid. Equation (8.24) thus implies $\delta S / S=0$, in agreement with the adiabaticity of the fluctuations.

After having computed the corrections induced by the magnetic field on the (leading) adiabatic initial condition the temperature anisotropies can be 
computed using the Sachs-Wolfe effect 292. In terms of the gauge-invariant variables introduced in the present analysis, the various contributions to the Sachs-Wolfe effect, along the $\vec{n}$ direction, can be written as

$$
\frac{\delta T}{T}\left(\vec{n}, \eta_{0}, x_{0}\right)=\left[\frac{\delta_{\mathrm{r}}}{4}+\vec{n} \cdot \vec{\nabla} v_{\mathrm{b}}+\Phi\right]\left(\eta_{\mathrm{dec}}, \vec{x}\left(\eta_{\mathrm{dec}}\right)\right)-\int_{\eta_{0}}^{\eta_{\mathrm{dec}}}\left(\Phi^{\prime}+\Psi^{\prime}\right)(\eta, \vec{x}(\eta)) d \eta
$$

where $\eta_{0}$ is the present time, and $\vec{x}(\eta)=\vec{x}_{0}-\vec{n}\left(\eta-\eta_{0}\right)$ is the unperturbed photon position at the time $\eta$ for an observer in $\vec{x}_{0}$. The term $\vec{v}_{\mathrm{b}}$ is the peculiar velocity of the baryonic matter component. In Fig. 11] the $C_{\ell}$ are plotted

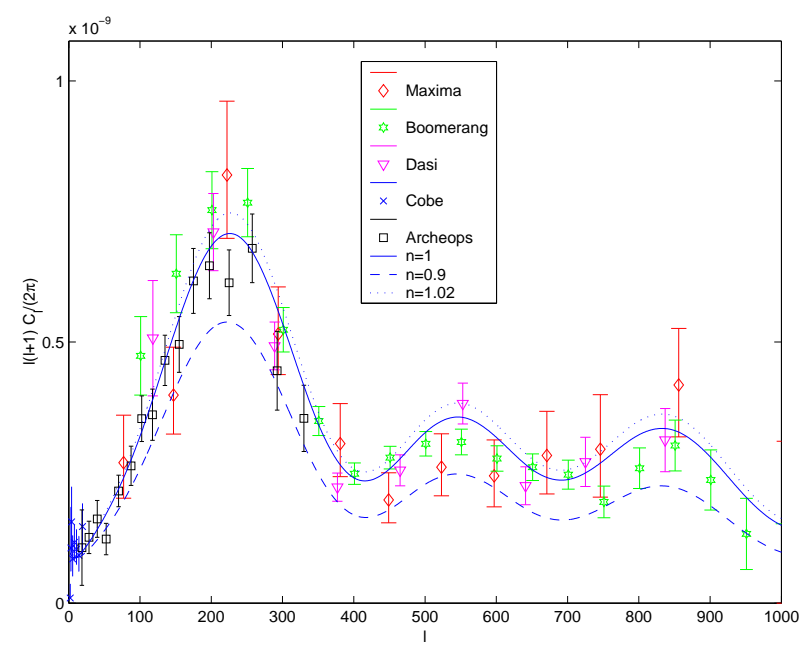

Figure 11: The spectrum of $C_{\ell}$ is illustrated for a fiducial set of parameters $\left(h_{0}=0.65, \Omega_{\mathrm{b}}=0.04733, \Omega_{\Lambda}=0.7, \Omega_{\mathrm{m}}=0.25267\right.$ ) and for flat (full line, $n=1$ ), slightly red (dashed line, $n=0.9$ ) and slightly blue (dotted line, $n=1.02$ ) spectral indices for the constant (adiabatic) mode.

in the case of models with adiabatic initial conditions. The data points reported in Figs. 11] and 12 are those from COBE [293, 294], BOOMERANG [295], DASI [296], MAXIMA [297] and ARCHEOPS 298. Notice that the data reported in 298. fill the "gap" between the last COBE points and the points of [295, 296, 297]. In Fig. 12 the recent WMAP data are reported [299, 300, 301]. 


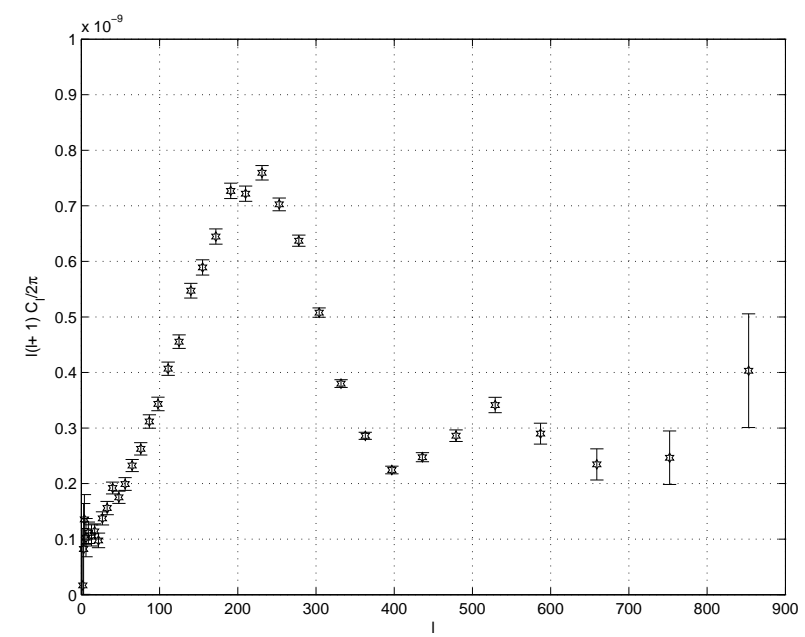

Figure 12: The WMAP data are reported.

Recall, that the temperature fluctuations are customarily discussed in terms of their Legendre transform

$$
\frac{\delta T}{T}\left(\vec{x}_{0}, \vec{n}, \eta_{0}\right)=\sum_{\ell, m} a_{\ell m}\left(\vec{x}_{0}\right) Y_{\ell m}(\vec{n}),
$$

where the coefficients $a_{\ell m}$ define the angular power spectrum $C_{\ell}$ by

$$
\left\langle a_{\ell m} \cdot a_{\ell^{\prime} m^{\prime}}^{*}\right\rangle=\delta_{\ell \ell^{\prime}} \delta_{m m^{\prime}} C_{\ell},
$$

and determine the two-point (scalar) correlation function of the temperature fluctuations, namely

$$
\begin{aligned}
\left\langle\frac{\delta T}{T}(\vec{n}) \frac{\delta T}{T}\left(\vec{n}^{\prime}\right)\right\rangle_{\left(\vec{n} \cdot \vec{n}^{\prime}=\cos \vartheta\right)} & =\sum_{\ell \ell^{\prime} m m^{\prime}}\left\langle a_{\ell m} a_{\ell^{\prime} m^{\prime}}^{*}\right\rangle Y_{\ell m}(\vec{n}) Y_{\ell^{\prime} m^{\prime}}^{*}\left(\overrightarrow{n^{\prime}}\right) \\
& =\frac{1}{4 \pi} \sum_{\ell}(2 \ell+1) C_{\ell} P_{\ell}(\cos \vartheta) .
\end{aligned}
$$

In Fig. 11 and 12 the experimental points are presented in terms of the $C_{\ell}$ spectra.

In Fig. 11 and 12 the curves fitting the data are obtained by imposing adiabatic initial conditions. If magnetic fields would seed directly the CMB 
anisotropies a characteristic "hump" would appear for $\ell<100$ which is not compatible with the experimental data. If, on the contrary, the condition (8.23) holds, then the modifications due to a magnetic field can be numerically computed for a given magnetic spectral index and for a given amplitude. This analysis has been performed by Koh and Lee 302. The claim is that by modifying the amplitude of the magnetic field in a way compatible with the cosmological constraint the effect on the scalar $C_{\ell}$ can be as large as the $10 \%$ for a given magnetic spectral index.

The main problem is order to detect large-scale magnetic fields from the spectrum of temperature fluctuations is the parameter space. On top of the usual parameters common in the CMB analysis at least two new parameters should be introduced, namely the magnetic spectral index and the amplitude of the magnetic field. The proof of this statement is that there is no available analysis of the WMAP data including also the presence of large scale magnetic fields in the initial conditions according to the lines presented in this Section.

To set bounds on primordial magnetic fields from CMB anisotropies it is often assumed that the magnetic field is fully homogeneous. In this case the magnetic field amplitude has been bounded to be $B \leq 10^{-3} \mathrm{G}$ at the decoupling time [303. (see also 305] for a complementary analysis always in the case of a homogeneous magnetic field).

There is the hope that since magnetic fields contribute not only to the scalar fluctuations, but also to the vector and tensor modes of the geometry useful informations cann be also obtained from the analysis of vector and tensor power spectra. In 304 temperature and polarization power spectra induced by vector and tensor perturbations have been computed by assuning a power-law spectrum of magnetic inhomogeneities. In [306, 307, 308, the tensor modes of the geometry have been specifically investigated. In [309, 310, 311 it is argued that magnetic fields can induce anisotropies in the polarization for rather small length scales, i.e. $\ell>1000$.

As already pointed out in the present Section, magnetic fields may be assumed to be force free in various cases. This is an approximation which may also be relaxed. However, even if magnetic fields are assumed to be force-free there is no reason to assume that their associated magnetic helicity (or gyrotropy) vanishes. In 312 the posssible effects of helical magnetic fields on CMB physics have been investigated. If helical flows are present at recombination, they would produce parity violating temperature-polarization correlations. The magnitude of helical flows induced by helical magnetic 
fields turns out to be unobservably small, but better prospects of constraining helical magnetic fields come from maps of Faraday rotation measurements of the CMB 312.

\subsection{Faraday rotation of CMB}

Large scale magnetic fields present at the decoupling epoch can also depolarize CMB 313. The polarization of the CMB represents a very interesting observable which has been extensively investigated in the past both from the theoretical 314 and experimental points of view 315, 316]. Forthcoming satellite missions like PLANCK [317] seem to be able to achieve a level of sensitivity which will enrich decisively our experimental knowledge of the CMB polarization with new direct measurements.

If the background geometry of the universe is homogeneous but not isotropic the CMB is naturally polarized 314. This phenomenon occurs, for example, in Bianchi-type I models 318. On the other hand if the background geometry is homogeneous and isotropic (like in the Friedmann-RobertsonWalker case) it seems very reasonable that the CMB acquires a small degree of linear polarization provided the radiation field has a non-vanishing quadrupole component at the moment of last scattering [319, 320].

Before decoupling photons, baryons and electrons form a unique fluid which possesses only monopole and dipole moments, but not quadrupole. Needless to say, in a homogeneous and isotropic model of FRW type a possible source of linear polarization for the CMB becomes efficient only at the decoupling and therefore a small degree of linear polarization seems a firmly established theoretical option which will be (hopefully) subjected to direct tests in the near future. The linear polarization of the CMB is a very promising laboratory in order to directly probe the speculated existence of a large scale magnetic field (coherent over the horizon size at the decoupling) which might actually rotate (through the Faraday effect) the polarization plane of the CMB.

Consider, for instance, a linearly polarized electromagnetic wave of physical frequency $\omega$ travelling along the $\hat{x}$ direction in a plasma of ions and electrons together with a magnetic field $(\vec{B})$ oriented along an arbitrary direction ( which might coincide with $\hat{x}$ in the simplest case). If we let the polarization vector at the origin $(x=y=z=0, t=0)$ be directed along the $\hat{y}$ axis, after the wave has traveled a length $\Delta x$, the corresponding angular 
shift $(\Delta \alpha)$ in the polarization plane will be :

$$
\Delta \alpha=f_{e} \frac{e}{2 m}\left(\frac{\omega_{\mathrm{p}}}{\omega}\right)^{2}(\vec{B} \cdot \hat{x}) \Delta x
$$

From Eq. (8.30) by stochastically averaging over all the possible orientations of $\vec{B}$ and by assuming that the last scattering surface is infinitely thin (i.e. that $\Delta x f_{e} n_{e} \simeq \sigma_{T}^{-1}$ where $\sigma_{T}$ is the Thompson cross section) we get an expression connecting the RMS of the rotation angle to the magnitude of $\bar{B}$ at $t \simeq t_{d e c}$

$$
\begin{aligned}
& \left\langle(\Delta \alpha)^{2}\right\rangle^{1 / 2} \simeq 1.6^{0}\left(\frac{B\left(t_{d e c}\right)}{B_{c}}\right)\left(\frac{\omega_{M}}{\omega}\right)^{2}, \\
& B_{c}=10^{-3} \mathrm{G}, \quad \omega_{M} \simeq 3 \times 10^{10} \mathrm{~Hz}
\end{aligned}
$$

(in the previous equation we implicitly assumed that the frequency of the incident electro-magnetic radiation is centered around the maximum of the CMB). We can easily argue from Eq. (8.31) that if $B\left(t_{d e c}\right) \geq B_{c}$ the expected rotation in the polarization plane of the CMB is non negligible. Even if we are not interested, at this level, in a precise estimate of $\Delta \alpha$, we point out that more refined determinations of the expected Faraday rotation signal (for an incident frequency $\omega_{M} \sim 30 \mathrm{GHz}$ ) were recently carried out 321, 322 leading to a result fairly consistent with (8.30).

Then, the statement is the following. If the CMB is linearly polarized and if a large scale magnetic field is present at the decoupling epoch, then the polarization plane of the CMB can be rotated [313]. The predictions of different models can then be confronted with the requirements coming from a possible detection of depolarization of the CMB [313].

\subsection{Relic gravitational waves}

Magnetic fields can source the evolution equations of the fluctuations of the geometry also over length-scales much smaller than the ones where CMB anisotropy experiments are conduced. This suggests that magnetic inhomogeneities may leave an imprint on the relc background of gravitational waves.

If a hypermagnetic background is present for $T>T_{c}$, then, as discussed in Section 5 and 6 , the energy momentum tensor will acquire a small anisotropic component which will source the evolution equation of the tensor fluctuations of the metric. Suppose now, that $\left|\overrightarrow{\mathcal{H}}_{Y}\right|$ has constant amplitude and that it is 
also homogeneous. Then as argued in [323] we can easily deduce the critical fraction of energy density present today in relic gravitons of EW origin

$$
\Omega_{\mathrm{gw}}\left(t_{0}\right)=\frac{\rho_{\mathrm{gw}}}{\rho_{c}} \simeq z_{\mathrm{eq}}^{-1} r^{2}, \quad \rho_{c}\left(T_{c}\right) \simeq N_{\mathrm{eff}} T_{c}^{4}
$$

( $z_{\mathrm{eq}}$ is the redshift from the time of matter-radiation, equality). Because of the structure of the AMHD equations, stable hypermagnetic fields will be present not only for $\omega_{\text {ew }} \sim k_{\text {ew }} / a$ but for all the range $\omega_{\text {ew }}<\omega<\omega_{\sigma}$ where $\omega_{\sigma}$ is the diffusivity frequency. Let us assume, for instance, that $T_{c} \sim 100$ $\mathrm{GeV}$ and $g_{*}=106.75$. Then, the (present) values of $\omega_{\text {ew }}$ is

$$
\omega_{\mathrm{ew}}\left(t_{0}\right) \simeq 2.01 \times 10^{-7}\left(\frac{T_{c}}{1 \mathrm{GeV}}\right)\left(\frac{g_{*}}{100}\right)^{1 / 6} \mathrm{~Hz}
$$

Thus, $\omega_{\sigma}\left(t_{0}\right) \sim 10^{8} \omega_{\text {ew }}$. Suppose now that $T_{c} \sim 100 \mathrm{GeV}$; than we will have that $\omega_{\text {ew }}\left(t_{0}\right) \sim 10^{-5} \mathrm{~Hz}$. Suppose now, that

$$
\left|\overrightarrow{\mathcal{H}}_{Y}\right| / T_{c}^{2} \geq 0.3
$$

as, for instance, implied by the analysis of the electroweak phase diagram in the presence of a magnetized background. This requirement imposes $r \simeq 0.1-$ 0.001 and, consequently,

$$
h_{0}^{2} \Omega_{\mathrm{GW}} \simeq 10^{-7}-10^{-8} .
$$

Notice that this signal would occurr in a (present) frequency range between $10^{-5}$ and $10^{3} \mathrm{~Hz}$. This signal satisfies the presently available phenomenological bounds on the graviton backgrounds of primordial origin. The pulsar timing bound ( which applies for present frequencies $\omega_{P} \sim 10^{-8} \mathrm{~Hz}$ and implies $h_{0}^{2} \Omega_{\mathrm{GW}} \leq 10^{-8}$ ) is automatically satisfied since our hypermagnetic background is defined for $10^{-5} \mathrm{~Hz} \leq \omega \leq 10^{3} \mathrm{~Hz}$. The large scale bounds would imply $h_{0}^{2} \Omega_{\mathrm{GW}}<7 \times 10^{-11}$ but a at much lower frequency (i.e. $10^{-18}$ $\mathrm{Hz}$ ). The signal discussed here is completely absent for frequencies $\omega<\omega_{\text {ew }}$. Notice that this signal is clearly distinguishable from other stochastic backgrounds occurring at much higher frequencies ( $\mathrm{GHz}$ region) like the ones predicted by quintessential inflation 324, 325, 326. It is equally distinguishable from signals due to pre-big-bang cosmology (mainly in the window of ground based interferometers [327]). The frequency of operation of the interferometric devices (VIRGO/LIGO) is located between few $\mathrm{Hz}$ and $10 \mathrm{kHz}$ 
[327. The frequency of operation of LISA is well below the $\mathrm{Hz}$ (i.e. $10^{-3} \mathrm{~Hz}$, approximately). In this model the signal can be located both in the LISA window and in the VIRGO/LIGO window due to the hierarchy between the hypermagnetic diffusivity scale and the horizon scale at the phase transition. In Fig. 13 the full thick line illustrates the spectrum of relic gravitational

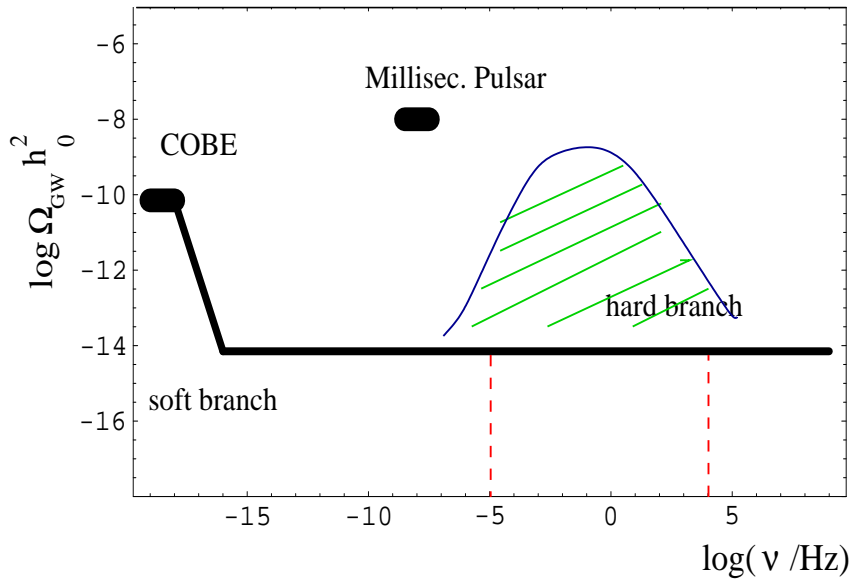

Figure 13: The stochastic background of GW produced by inflationary models with flat logarithmic energy spectrum, illustrated together with the GW background of hypermagnetic origin. The frequencies marked with dashed lines correspond to the electroweak frequency and to the hypermagnetic diffusivity frequency.

waves produced in a conventional model for the evolution of the universe. The flat plateau corresponds to modes which left the horizon during the inflationary stage of expansion and re-entered duriing the radiation dominated phase. The decreasing slope between $10^{-16}$ and $10^{-18} \mathrm{~Hz}$ is due to modes leaving the horizon during inflation and re-entering during the maatter dominated stage of expansion. Clearly, the signal provided by a background of hypermagnetic fields can be even 7 order of magnitude larger than the inflationary prediction. The interplay between gravitational waves and large-scale magnetic fields has been also the subject of recent interesting investigations 329, 330, 331. 


\section{Gravitating magnetic fields}

Up to now large scale magnetic fields have been treated in different frameworks but always within a perturbative approach. However, magnetic fields can also break explicitely the isotropy (but not the homogeneity) of the background geometry

Suppose that at some time $t_{1}$ the Universe becomes transparent to radiation and suppose that, at the same time, the four-dimensional background geometry (which we assume, for simplicity, spatially flat) has a very tiny amount of anisotropy in the scale factors associated with different spatial directions, namely

$$
d s^{2}=d t^{2}-a^{2}(t) d x^{2}-b^{2}(t)\left[d y^{2}+d z^{2}\right],
$$

where $b(t)$, as it will be clear in a moment, has to be only slightly different from $a(t)$. The electromagnetic radiation propagating along the $x$ and $y$ axes will have a different temperature, namely

$$
T_{x}(t)=T_{1} \frac{a_{1}}{a}=T_{1} e^{-\int H(t) d t}, \quad T_{y}(t)=T_{1} \frac{b_{1}}{b}=T_{1} e^{-\int F(t) d t},
$$

where $H(t)$ and $F(t)$ are the two Hubble factors associated, respectively with $a(t)$ and $b(t)$. The temperature anisotropy associated with the electromagnetic radiation propagating along two directions with different expansion rates can be roughly estimated, in the limit $H(t)-F(t) \ll 1$, as

$$
\frac{\Delta T}{T} \sim \int[H(t)-F(t)] d t=\frac{1}{2} \int \varepsilon(t) d \log t
$$

where, in the second equality, we assumed that the deviations from the radiation dominated expansion can be written as $F(t) \sim(1-\varepsilon(t)) / 2 t$ with $|\varepsilon(t)| \ll 1$. The function $\varepsilon$ can be connected with the shear parameter, i.e. $[H(t)-F(t)] /[H(t)+2 F(t)]$ which measures the anisotropy in the expansion. In the standard context [284, 285] Einstein equations are solved in the metric (9.1) with fluid sources and in the presence of a magnetic field. The shear parameter is then connected with the magnetic field intensity.

The dynamical origin of the primordial anisotropy in the expansion can be connected with the existence of a primordial magnetic field (not dynamically generated but postulated as an initial condition) or with some other sources of anisotropic pressure and, therefore, the possible bounds on the 
temperature anisotropy can be translated into bounds on the time evolution of the anisotropic scale factors 303 .

Today the amount of anisotropy in the expansion must be very small because of the effect we just described. In the present Section some highly speculative considerations on the possible rôle of magnetic fields in the early Universe will be intrtoduced. In spite of the fact that magnetic fields must be sufficiently small today, in the past history of the Universe they might have been very large even modifying the dynamics of the geometry. During the magnetic phase the anisotropy in the expansion will be constant (or even grow). However, after the magnetic phase a sufficienly long radiation dominated phase may isotropize the background leading to a tiny amount of anisotropy in the expansion. This is the basic scenario invoked long ago by Zeldovich [284, 285].

In modern approaches to cosmology, the dynamics of the Universe at very high densities is often discussed in terms of the low-energy string effective action. It is then useful to analyze the Zeldovich proposal in terms of the modern perspective.

In the low energy limit, the dilaton field is directly coupled to the kinetic term of the Maxwell field 332.

$$
S=-\frac{1}{2 \lambda_{s}^{2}} \int d^{4} x \sqrt{-g} e^{-\varphi}\left[R+g^{\alpha \beta} \partial_{\alpha} \varphi \partial_{\beta} \varphi-\frac{1}{12} H_{\mu \nu \alpha} H^{\mu \nu \alpha}+\frac{1}{4} F_{\alpha \beta} F^{\alpha \beta}+\ldots\right]
$$

where $F_{\alpha \beta}=\nabla_{[\alpha} A_{\beta]}$ is the Maxwell field strength and $\nabla_{\mu}$ is the covariant derivative with respect to the String frame metric $g_{\mu \nu}$. Notice that $H_{\mu \nu \alpha}$ is the antisymmetric field strength. In Eq. (9.4) the ellipses stand for a possible (non-perturbative) dilaton potential and for the string tension corrections parameterized by $\alpha^{\prime}=\lambda_{s}^{2}$. In Eq. (9.4) $F_{\mu \nu}$ can be thought as the Maxwell field associated with a $U(1)$ subgroup of $E_{8} \times E_{8}$.

Consider a spatially flat background configuration with vanishing antysimmetric field strangth $\left(H_{\mu \nu \alpha}=0\right)$ and vanishing dilaton potential. The dilaton depends only on time and the metric will be taken fully anisotropic since we want to study possible solutions with a homogeneous magnetic field which is expected to break the isotropy of the background:

$$
d s^{2}=g_{\mu \nu} d x^{\mu} d x^{\nu}=d t^{2}-a^{2}(t) d x^{2}-b^{2}(t) d y^{2}-c^{2}(t) d z^{2} .
$$

By varying the effective action with respect to $\varphi, g_{\mu \nu}$ and the vector 
potential $A_{\mu}$ we get, respectively

$$
\begin{aligned}
& R-g^{\alpha \beta} \partial_{\alpha} \varphi \partial_{\beta} \varphi+2 g^{\alpha \beta} \nabla_{\alpha} \nabla_{\beta} \varphi=-\frac{1}{4} F_{\alpha \beta} F^{\alpha \beta} \\
& R_{\mu}^{\nu}-\frac{1}{2} \delta_{\mu}^{\nu} R=\frac{1}{2}\left[\frac{1}{4} \delta_{\mu}^{\nu} F_{\alpha \beta} F^{\alpha \beta}-F_{\mu \beta} F^{\nu \beta}\right] \\
& -\frac{1}{2} \delta_{\mu}^{\nu} g^{\alpha \beta} \partial_{\alpha} \varphi \partial_{\beta} \varphi-\nabla_{\mu} \nabla^{\nu} \varphi+\delta_{\mu}^{\nu} \frac{1}{\sqrt{-g}} \partial_{\alpha}\left[\sqrt{-g} g^{\alpha \beta} \partial_{\beta} \varphi\right], \\
& \nabla_{\mu}\left[e^{-\varphi} F^{\mu \nu}\right]=0 .
\end{aligned}
$$

where $\nabla_{\mu}$ denotes covariant differentiation with respect to the metric of Eq. (9.5). Inserting Eq. (5.58) into Eq. (9.7) we get that Eq. (9.7) can be expressed as

$$
R_{\mu}^{\nu}+\nabla_{\mu} \nabla^{\nu} \varphi+\frac{1}{2} F_{\mu \alpha} F^{\nu \alpha}=0
$$

Consider now a homogeneous magnetic field directed along the $x$ axis. The generalized Maxwell equations (9.8) and the associated Bianchi identities can then be trivially solved by the field strength $F_{y z}=-F_{z y}$. The resulting system of equations (5.58), (9.9) can than be written, in the metric of Eq. (9.5), as

$$
\begin{aligned}
& \ddot{\varphi}=H^{2}+F^{2}+G^{2}+\dot{H}+\dot{F}+\dot{G}, \\
& H \dot{\varphi}=H F+H G+H^{2}+\dot{H}, \\
& F \dot{\varphi}=H F+F G+F^{2}+\dot{F}-\frac{B^{2}}{2 b^{2} c^{2}}, \\
& G \dot{\varphi}=G H+F G+G^{2}+\dot{G}-\frac{B^{2}}{2 b^{2} c^{2}},
\end{aligned}
$$

where Eqs. (9.10)-(9.13) correspond, respectively to the (00) and (ii) components of Eq. (9.9).

The solution of Eqs. (9.10)- (9.13)

$$
\begin{aligned}
& a(\eta)=a_{0} e^{\mathcal{H}_{0} \eta}, \quad b(\eta)=b_{0} e^{\varphi_{0}} e^{\left(\mathcal{F}_{0}+\mathcal{H}_{0}+\Delta_{0}\right) \eta}\left[e^{-2 \Delta_{0} \eta}+e^{\varphi_{1}}\right] \\
& c(\eta)=c_{0} e^{\phi_{0}} e^{\left(\mathcal{G}_{0}+\mathcal{H}_{0}+\Delta_{0}\right) \eta}\left[e^{-2 \Delta_{0} \eta}+e^{\varphi_{1}}\right] \\
& \varphi(\eta)=\varphi_{0}+\left(\mathcal{H}_{0}+\Delta_{0}\right) \eta+\log \left[e^{\varphi_{1}}+e^{-2 \Delta_{0} \eta}\right]
\end{aligned}
$$


where

$$
\Delta_{0} \equiv \sqrt{\mathcal{H}_{0}^{2}+\Lambda_{0}}=\frac{a_{0}}{2 \sqrt{2}} e^{-\left(\varphi_{0}+\varphi_{1}\right)} B .
$$

The solution given in Eq.(9.16) s given in terms of a new variable (a generalized conformal time) defined by the following differential relation

$$
e^{-\bar{\varphi}} d \eta=d t, \quad \bar{\varphi}=\varphi-\log a b c .
$$

These solutions represent the generalization of pre-big bang solutions [123] to the case where a constant magnetic field is included. Generalizations of the solution (9.16) can be obtained in various models anisotropic (but homogeneous) models of the Bianchi class [337] like Bianchi I, II, III, $\mathrm{VI}_{-1}$ and $\mathrm{VII}_{0}$ according to the usual classification which can be found, for instance, in 333. Further generalizations of the solution (9.16) are possible in the Kantowski-Sachs metric with a magnetic field aligned alond the radial coordinate [335, 336].

Magnetized cosmological solutions can be used in order to describe some early phase in the life of the universe. This highly speculative idea may be implemented in semi-realistic models provided the large anisotropy in the expansion decays at later epochs. In the standard context [284, 285] the only source of isotropization is the evolution in a radiation dominated background since the shear parameter decays if the background is dominated by radiation. If quadratic corrections are present, an initially anisotropic solution becomes isotropic as a consequence of the dynamical properties of the evolution equations as discussed in detail in 332 .

Gauge fields have also been studied as a source of inflationary evolution by Ford [338] and later by Lidsey [339, 340]. The gauge kinetic term is supplemented by a potential which is a function of $A_{\mu} A^{\mu}$. Both exponential inflation [338] and power-law inflation [339] can be realized in these models.

\section{Concluding Remarks}

The topics reported in the present review suggest various open problems on the nature of large-scale magnetic fields in the present and in the early Universe:

- in principle the morphology of magnetic fields in spiral galaxies could be used in order to understand the origin of galactic magnetism but in practice the observations offer answers which are often contradictory; 
- recent observations of magnetic fields inside Abell clusters confirm the presence of strong magnetic fields in the $\mu \mathrm{G}$ range;

- there is evidence that also superclusters are magnetized;

- in spite of the fact that magnetic fields may modify the patterns of the CMB anisotropies and, eventually, induce anisotropies in the polarization, the theoretical analysis has been rarely corroborated by the comparison with the available experimental data;

- Faraday rotation of the CMB polarization would be a powerful tool to study the possible exsitence of large scale magnetic fields prior to recombination;

- the imprints of magnetic fields on the relic background of gravitational waves (in the window of wide band interferometers) allows to test the existence of a background of Abelian gauge fields at the electroweak epoch.

It would be important, for the theorist, a reasonable accuracy (within one order of magnitude) in the experimental determination of large-scale magnetic fields. For instance even one order of magnitude in the intensity of the intracluster magnetic field (i.e. $10^{-6} \mathrm{G}$ rather than $10^{-7} \mathrm{G}$ ) makes a difference as far as the problem of the origin is concerned. In the same perspective, more accurate determinations of the typical correlation scales of the intra-cluster fields would be desirable.

There is, at the moment, no compelling reason why large-scale magnetic fields should not be primordial, at least to some degree:

- in the early Universe magnetic fields are easily produced during phase transitions but their typical correlation scales are small;

- inflation greatly helps in producing correlations over very large scales and the (early) variation of gauge couplings during the inflationary phase allows the generation of intense large-scale fields;

- primordial magnetic fields may have an impact on the thermodynamical history of the Universe, but, in practice, the obtained constraints improve only marginally, in various interesting cases, the critical density bound imposed on the magnetic energy density; 
- if magnetic fields are generated during phase transitions, a growth in their correlation scale cannot be excluded because of turbulent phenomena whose existence can be reasonably expected (but not firmly predicted) on the basis of our terrestrial knowledge of magnetized plasmas.

In the next decade a progress of the empirical knowledge is expected in apparently unrelated areas like $x$ and $\gamma$-ray astronomy, radio-astronomy, CMB physics, detection of relic gravitational waves. All these areas are connected with the existence and with the properties of large-scale magnetic fields in the early and in the present Universe. This connection is sometimes rather fragile since it is mediated by various theoretical assumptions. However, there is the hope that, in a not too distant future, some of the puzzles related to the origin and existence of large-scale magnetic fields may be resolved and some of the current theoretical guessworks firmly ruled out.

\section{Acknowledgements}

Some of the ideas presented in this review have been elaborated and assembled on the occasion of different sets of lectures delivered through the years. The author wishes to express his gratitude to D. Babusci, H. de Vega, M. Gasperini, H. Kurki-Suonio, E. Keihänen, N. Sanchez, M. Shaposhnikov, G. Veneziano, A. Vilenkin. A special thank is due to G. Cocconi for important remarks which improved the first draft. 


\section{A Complements on MHD description}

\section{Two fluids and one-fluid MHD equations}

In a two-fluid plasma description the charge carriers are the ions (for simplicity we can think of them as protons) and the electrons. The two fluid equations treat the ions and the electrons as two conducting fluids which are coupled as in Eqs. (4.8)-(4.12). Given the two- fluid description, one-fluid variables can be defined directly in terms of the two-fluid variables

$$
\begin{aligned}
& \rho_{\mathrm{m}}(\vec{x}, t)=m_{e} n_{e}(\vec{x}, t)+m_{p} n_{p}(\vec{x}, t), \\
& \rho_{\mathrm{q}}(\vec{x}, t)=e\left[n_{p}(\vec{x}, t)-n_{e}(\vec{x}, t)\right], \\
& \vec{J}=e\left[n_{p} \vec{v}_{p}-n_{e} \vec{v}_{e}\right] .
\end{aligned}
$$

In the case of a globally neutral plasma $n_{e} \sim n_{p}=n_{q}$ and $\rho_{q}=0$. The ion and electron equations then become

$$
\begin{aligned}
& m_{p} n_{q}\left[\frac{\partial}{\partial t}+\vec{v}_{\mathrm{p}} \cdot \vec{\nabla}\right] \vec{v}_{\mathrm{p}}=e n_{q}\left(\vec{E}+\vec{v}_{p} \times \vec{B}\right)-\vec{\nabla} p_{p}+\mathcal{C}_{p e} \\
& m_{e} n_{q}\left[\frac{\partial}{\partial t}+\vec{v}_{\mathrm{e}} \cdot \vec{\nabla}\right] \vec{v}_{\mathrm{e}}=-e n_{q}\left(\vec{E}+\vec{v}_{e} \times \vec{B}\right)-\vec{\nabla} p_{e}+\mathcal{C}_{e p}
\end{aligned}
$$

where $\mathcal{C}_{p e}$ and $\mathcal{C}_{p e}$ are the collision terms. In the globally neutral case the center of mass velocity becomes

$$
\vec{v}=\frac{m_{p} \vec{v}_{p}+m_{e} \vec{v}_{e}}{m_{p}+m_{e}},
$$

and the one-fluid mass and charge density conservations become

$$
\begin{aligned}
& \frac{\partial \rho_{\mathrm{m}}}{\partial t}+\vec{\nabla} \cdot\left(\rho_{\mathrm{m}} \vec{v}\right)=0, \\
& \frac{\partial \rho_{q}}{\partial t}+\vec{\nabla} \cdot \vec{J}=0 .
\end{aligned}
$$

Summing up Eqs. (A.2) and (A.3) leads, with some algebra involving the continuity equation, to the momentum transport equation in the one-fluid theory:

$$
\rho_{\mathrm{m}}\left[\frac{\partial \vec{v}}{\partial t}+\vec{v} \cdot \vec{\nabla}\right] \vec{v}=\vec{J} \times \vec{B}-\vec{\nabla} P
$$


where $P=P_{e}+P_{i}$. In Eq. (A.7) the collision term vanishes if there are no neutral particles, i.e. if the plasma is fully ionized. The final equation of the one-fluid description is obtained by taking the difference of Eqs. (A.2) and (A.3) after having multiplied Eq. (A.2) by $m_{e}$ and Eq. (A.3) by $m_{p}$. This procedure is more tricky and it is discussed in standard textbooks of plasma physics 85, 86. The key points in the derivation are that the limit for $m_{e} / m_{p} \rightarrow 0$ must be taken. The problem with this procedure is that the subtraction of the two mentioned equations does not guarantee that viscous and collisional effects are negligible. The result of this procedure is the socalled one-fluid generalized Ohm law:

$$
\vec{E}+\vec{v} \times \vec{B}=\frac{1}{\sigma} \vec{J}+\frac{1}{e n_{q}}\left(\vec{J} \times \vec{B}-\vec{\nabla} P_{e}\right) .
$$

The term $\vec{J} \times B$ is nothing but the Hall current and $\vec{\nabla} P_{e}$ is often called thermoelectric term. Finally the term $\vec{J} / \sigma$ is the resistivity term and $\sigma$ is the conductivity of the one-fluid description. In Eq. (A.8) the pressure has been taken to be isotropic. This is, however, not a direct consequence of the calculation presented in this Appendix but it is an assumption which may (and should) be relaxed in some cases. In the plasma physics literature 85 , 86. the anisotropic pressure contribution is neglected for the simple reason that experiments terrestrial plasmas show that this terms is often negligible.

\section{Conservation laws in resistive MHD}

Consider and arbitrary closed surface $\Sigma$ which moves with the plasma. Then, by definition of the bulk velocity of the plasma ( $\vec{v}$ we can also write $d \vec{\Sigma}=$ $\vec{v} \times d \vec{l} d \eta$. The (total) time derivative of the flux can therefore be expressed as

$$
\frac{d}{d t} \int_{\Sigma} \vec{B} \cdot d \vec{\Sigma}=\int_{\Sigma} \frac{\partial \vec{B}}{\partial t} \cdot d \vec{\Sigma}+\int_{\partial \Sigma} \vec{B} \times \vec{v} \cdot d \vec{l}
$$

where $\partial \Sigma$ is the boundary of $\Sigma$. Using now the properties of the vector products (i.e. $\vec{B} \times \vec{v} \cdot d \vec{l}=-\vec{v} \times \vec{B} \cdot d \vec{l}$ ) we can express $\vec{v} \times \vec{B}$ though the Ohm law given in Eq. (4.23) and we obtain that

$$
\vec{v} \times \vec{B} \simeq-\vec{E}+\frac{1}{4 \pi \sigma} \vec{\nabla} \times \vec{B}
$$


Using now Eq. (A.9) together with the Stokes theorem, the following expression can be obtained

$$
\frac{d}{d t} \int_{\Sigma} \vec{B} \cdot d \vec{\Sigma}=\int_{\Sigma}\left[\frac{\partial \vec{B}}{\partial t}+\vec{\nabla} \times \vec{E}\right] \cdot d \vec{\Sigma}-\frac{1}{4 \pi \sigma} \int_{\Sigma} \vec{\nabla} \times \vec{\nabla} \times \vec{B} \cdot d \vec{\Sigma} .
$$

From the Maxwell's equations the first part at the right hand side of Eq. (A.11) is zero and Eq. (4.24), expressing the Alfvén theorem, is recovered.

With similar algebraic manipulations (involving the use of various vector identities), the conservation of the magnetic helicity can be displayed. Consider a closed volume in the plasma, then we can write that $d V=d^{3} x=$ $\vec{v}_{\perp} \cdot d \vec{\Sigma} d \eta \equiv \vec{n} \cdot \vec{v}_{\perp} d \Sigma d \eta$ where $\vec{n}$ is the unit vector normal to $\Sigma$ (the boundary of $\mathrm{V}$, i.e. $\Sigma=\partial V$ ) and $\vec{v}_{\perp}$ is the component of the bulk velocity orthogonal to $\partial V$. The (total) time derivative of the magnetic helicity can now be written as

$$
\frac{d}{d \eta} \mathcal{H}_{M}=\int_{V} d^{3} x \frac{\partial}{\partial \eta}[\vec{A} \cdot \vec{B}]+\int_{\partial V=\Sigma} \vec{A} \cdot \vec{B} \vec{v}_{\perp} \cdot \vec{n} d \Sigma
$$

The partial derivative at the right hand side of Eq. (A.12 can be made explicit. Then the one-fluid MHD equations should be used recalling that the relation between the electromagnetic fields and the vector potential, for instance in the Coulomb gauge. Finally using again the Ohm law and transforming the obtained surface integrals into volume integrals (through the divergence theorem) Eq. (4.27), expressing the conservation of the helicity, can be obtained.

In spite of the fact that the conservation of the magnetic helicity can be derived in a specific gauge, the magnetic helicity is indeed a gauge invariant quantity. Consider a gauge transformation

$$
\vec{A} \rightarrow \vec{A}+\vec{\nabla} \xi
$$

then the magnetic helicity changes as

$$
\int_{V} d^{3} x \vec{A} \cdot \vec{B} \rightarrow \int_{V} d^{3} x \vec{A} \cdot \vec{B}+\int_{V} d^{3} x \vec{\nabla} \cdot[\xi \vec{B}]
$$

(in the second term at the right hand side we used the fact that the magnetic field is divergence free). By now using the divergence theorem we can express the volume integral as

$$
\int_{V} d^{3} x \vec{\nabla} \cdot[\xi \vec{B}]=\int_{\partial V=\Sigma} \xi \vec{B} \cdot \vec{n} d \Sigma .
$$


Now if, as we required, $\vec{B} \cdot \vec{n}=0$ in $\partial V$, the integral is exactly zero and $\mathcal{H}_{M}$ is gauge invariant. The condition $\vec{B} \cdot \vec{n}=0$ is not specific of a particular profile of the magnetic field. It can be always achieved by slicing the volume of integration in small flux tubes where, by definition, the magnetic field is orthogonal to the walls of the flux tube. 


\section{References}

[1] H. Alfvén, Arkiv. Mat. F. Astr., o. Fys. 29 B, 2 (1943).

[2] E. Fermi, Phys. Rev.75, 1169 (1949).

[3] H. Alfvén, Phys. Rev.75, 1732 (1949).

[4] W. A. Hiltner, Science109, 165 (1949).

[5] J. S. Hall, Science109, 166 (1949).

[6] L. J. Davis and J. L. Greenstein, Astrophys. J.114, 206 (1951).

[7] E. Fermi and S. Chandrasekar Astrophys. J.118, 113 (1953).

[8] E. Fermi and S. Chandrasekar Astrophys. J.118, 116 (1953).

[9] R. D. Richtmyer and E. Teller Phys. Rev. 75, 1729 (1949).

[10] R. Wielebinski and J. Shakeshaft, Nature195, 982 (1962).

[11] A. Lyne and F. Smith, Nature218, 124 (1968).

[12] C. Heiles, Annu. Rev. Astron. Astrophys.14, 1 (1976).

[13] P. P. Kronberg, Rep. Prog. Phys.57, 325 (1994).

[14] J. Vallée, Fundam. Cosmic Phys.19, 1 (1997).

[15] R. Beck, A. Brandenburg, D. Moss, A. Skhurov, and D. Sokoloff Annu. Rev. Astron. Astrophys.34, 155 (1996).

[16] E. Battaner and E. Florido, Fund. of Cosm. Phys.21, 1 (2000).

[17] J.-L. Han and R. Wieblinski, Chin. J. Astron. Astrophys. 2293 (2002).

[18] E. Zweibel and C. Heiles, Nature385, 131 (1997).

[19] Ya. B. Zeldovich, A. A. Ruzmaikin, and D.D. Sokoloff, Magnetic Fields in Astrophysics (Gordon and Breach Science, New York, 1983).

[20] E. N. Parker, Cosmical Magnetic Fields (Clarendon Press, Oxford, 1979). 
[21] A. A. Ruzmaikin, A. M. Shukurov, and D. D. Sokoloff Magnetic Fields of Galaxies, (Kluwer Academic Publisher, Dordrecht, 1988).

[22] J. Vallée, Fundam. Cosmic Phys.19, 319 (1998).

[23] D. Fiebig and R. Guensten, Astron. and Astrophys. 214, 333 (1989).

[24] R. Crutcher et al. Astrophys. J.514, L121 (1999).

[25] I. Shklovskij, Dokl. Akad. Nauk. USSR90, 983 (1953).

[26] X. Chi and A. W. Wolfendale, Nature362, 610 (1993).

[27] V. R. Buczilowski and R. Beck, Astron. Astrophys.241, 46 (1991)

[28] E. Hummel and R. Beck, Astron. Astrophys.303, 691 (1995).

[29] K. D. Lawson et al., MNRAS225, 307 (1987).

[30] J.-L. Han and G. J. Qiao, Astron. Asrtophys.288 759 (1994).

[31] J.-L. Han, R. Manchester, and G. Qiao Mon. Not. R. Astron. Soc.306, 371 (1999).

[32] P. Dineen and P. Coles, e-print Archive astro-ph/0306529 (to appear in Mon. Not. R. Astron. Soc.).

[33] O. Goldshmidt and Y. Rephaeli, Astrophys. J.411, 518 (1993).

[34] A. Crusius-Wätzel, P. Biermann, R. Schlickeiser, and I. Lerche, Astrophys. J.360, 417 (1990).

[35] W. Newmann, A. Newmann, and Y. Rephaeli, Astrophys. J.575, 755 (2002).

[36] C. Vogt and T. Ensslin, e-print Archive astro-ph/0302426 (to appear in Astronomy and Astrophysics).

[37] C. Vogt and T. Ensslin, e-print Archive astro-ph/0309441 (to appear in Astronomy and Astrophysics).

[38] T. Kolatt, Astrophys. J495, 564 (1998).

[39] D. Mathewson and V. Ford, Mem. R. Astron. Soc.74, 139 (1970). 
[40] R. N. Manchester, Astrophys. J.188, 637 (1974).

[41] R. Rand and A. Lyne, Mon. Not. R. Astron. Soc.268, 497 (1994).

[42] F. Yusef-Zadeh and M. Morris, Astrophys. J.320, 545 (1987).

[43] F. Yusef-Zadeh, D. Roberts, and M. Wardle, Astrophys. J. 490, L83 (1997).

[44] F. Yusef-Zadeh, M. Wardle, and P. Parastaran, Astrophys. J.475, L119 (1997).

[45] J. Vallée, Astrophys. J.566, 261 (2002).

[46] J. L. Han, R. N. Manchester, A. G. Lyne, and G. J. Qiao, Astrophys. J. 570, L17 (2002).

[47] Y. Sofue and M. Fujimoto, Astrophys. J.265, 722 (1983).

[48] D. Harari, S. Mollerach and E. Roulet, JHEP08 022 (1999).

[49] T. Stanev, Astrophys. J.479, 290 (1997).

[50] J. Han, Astrophys. and Space Sci. 278, 181 (2001).

[51] R. Rand and S. Kulkami, Astrophys. J. 343, 760 (1989)

[52] H. Ohno and S. Shibata, Mon. Not. R. Astron. Soc. 262, 953 (1993).

[53] T. Jones, D. Klebe, and J. Dickey, Astrophys. J. 458, 194 (1992).

[54] P. Biermann, J. Phys. G 23, 1 (1997).

[55] G. Farrar and T. Piran, Phys. Rev. Lett. 84, 3527 (2000).

[56] M. Giler, J. Wdowczyk and A. W. Wolfendale, J. Phys. G 6, 1561 (1980).

[57] K. Greisen, Phys. Rev. Lett. 16, 748 (1966).

[58] G. Zatsepin and V. Kuzmin, JETP Lett.4, 78 (1966).

[59] G. Medina Tanco, Astrophys. J. Lett.505, L79 (1998).

[60] P. Blasi and A. Olinto, Phys. Rev. D59, 023001 (1999). 
[61] G. Sigl, M. Lemoine, and P. Biermann, Astropart. Phys.10, 141 (1999).

[62] T. Stanev, D. Seckel, and R. Engel Phys. Rev. D68, 1030041.

[63] L. Widrow, Rev. Mod. Phys. 74, 775 (2002).

[64] M. Krause, R. Beck and E. Hummel Astron. and Astrophys. 217, 4 (1989).

[65] M. Krause, R. Beck and E. Hummel Astron. and Astrophys. 217, 17 (1989).

[66] R. Beck, Phil. Trans. R. Soc. Lond. A 358, 777 (2000).

[67] F. Krause and R. Beck, Astron. and Astrophys. 335, 789 (1998).

[68] M. Giovannini, Proc. of 7th Paris Cosmology Colloquium on High Energy Astrophysics for and from Space (eds. N. Sanchez and H. de Vega) e-Print Archive hep-ph/0208152.

[69] C. Carilli and G. Taylor Ann.Rev.Astron.Astrophys. 40, 319 (2002).

[70] K.-T. Kim, P.P. Kronberg, P. D. Dewdney and T. L. Landecker, Astrophys. J. 355, 29 (1990).

[71] L. Ferretti, D. Dallacasa, G. Giovannini, and A. Tagliani, Astronom. and Astrophys. 302, 680 (1995).

[72] O. Goldshmidt and Y. Raphaeli, Astrophys. J. 411, 518 (1993).

[73] T.E. Clarke, P.P. Kronberg and H. Böhringer, Astrophys. J. 547, L111 (2001).

[74] H. Böhringer, Rev. Mod. Astron. 8, 295 (1995).

[75] H. Hebeling, W. Voges, H. Böhringer, A. C. Edge, J. P. Huchra, and U. G. Briel Mon. Not. Astron. Soc. 281, 799 (1996).

[76] G. Giovannini and L. Ferretti, New Astronomy5, 335 (2000).

[77] H. Ohno et al., Astrophys.J. 584, 599 (2003).

[78] J. Vallée, Astron. J. 124, 1322 (2001). 
[79] K.-T. Kim, P. C. Tribble, and P.P. Kronberg, Astrophys. J. 379, 80 (1991).

[80] C. Isola and G. Sigl, Phys. Rev. D66, 083002 (2002).

[81] K. Dolag, D. Grasso, V. Springel, and Igor Tkachev, e-Print Archive astro-ph/0310912.

[82] A. Vlasov, Zh. Éksp. Teor. Fiz. 8, 291 (1938); J. Phys. 9, 25 (1945).

[83] L. D. Landau, J. Phys. U.S.S.R. 10, 25 (1945).

[84] E. M. Lifshitz and L. P. Pitaevskii, Physical Kinetics, (Pergamon Press, Oxford, England, 1980).

[85] N. A. Krall and A. W. Trivelpiece, Principles of Plasma Physics, (San Francisco Press, San Francisco 1986).

[86] F. Chen, Introduction to Plasma Physics, (Plenum Press, New York 1974).

[87] D. Biskamp, Non-linear Magnetohydrodynamics (Cambridge University Press, Cambridge, 1994).

[88] M. Giovannini, Phys. Rev. D58, 124027 (1998).

[89] S. I. Vainshtein and Ya. B. Zeldovich, Usp. Fiz. Nauk. 106, 431 (1972).

[90] W. H. Matthaeus, M. L. Goldstein, and S. R. Lantz, Phys. Fluids 29, 1504 (1986).

[91] J. Larmor, Rep. 87th Meeting Brit. Assoc. Adv. Sci. (John Murray, London 1919).

[92] J. H. Piddington, Aust. J. Phys. 23, 731 (1970).

[93] J. H. Piddington, Ap. and Sp. Sci. 35, 269 (1975).

[94] R. Kulsrud and S. Anderson, Astrophys. J. 396, 606 (1992).

[95] S. Vainshtein, Sov. Phys. JETP 34, 327 (1972).

[96] F. Cattaneo and S. Vainshtein, Astrophys. J. 376, L21 (1991). 
[97] R. M. Kulsrud, Annu. Rev. Astron. Astrophys. 37, 37 (1999).

[98] F. Krause and K. H. Rädler, Mean field Magnetohydrodynamics and Dynamo Theory, (Pergamon Press, Oxford, England 1980).

[99] R. Banerjee and K. Jedamzik, Phys. Rev. Lett91 , 251301 (2003)

[100] S. Weinberg, Gravitation and cosmology (Wiley, New York, 1971).

[101] P. Peebles, The Large Scale Structure of the Universe, (Princeton University Press, Princeton, New Jersey 1980).

[102] P. Peebles, Principles of Physical Csomology, (Princeton University Press, Princeton, New Jersey 1993).

[103] E. W. Kolb and M. S. Turner, The Early Universe (Addison-Wesley, Redwood City, CA, 1990).

[104] A. Guth, Phys.Rev.D23, 347 (1981).

[105] A. Albrecht and P. Steinhardt, Phys.Rev.Lett.48, 1220 (1982).

[106] A. Linde, Phys.Lett.B 108, 389 (1982).

[107] A. Linde, Phys.Lett.B129, 177 (1983).

[108] A. Linde, Phys.Rev.D 49, 748 (1994).

[109] J. Lidsey, et al., Rev.Mod.Phys.69, 373 (1997).

[110] H. de Vega and N. Sanchez, Phys. Lett. B 197, 320 (1987).

[111] H. de Vega and N. Sanchez, Phys. Rev. D 50, 7202 (1994).

[112] N. Sanchez and G. Veneziano, Nucl. Phys. B 333, 253 (1990).

[113] M. Gasperini, N. Sanchez and G. Veneziano, Nucl. Phys. B 364, 365 (1991).

[114] M. Gasperini, N. Sanchez and G. Veneziano, Int. J. Mod. Phys. A 6, 3853 (1991).

[115] M. Giovannini, Phys. Rev. D 55, 595 (1997). 
[116] J. D. Barrow, Phys. Rev. D 35, 1805 (1987).

[117] E. W. Kolb, M. J. Perry, and T.P. Walker, Phys. Rev. D 33, 869 (1986).

[118] F. S. Accetta, L. M. Krauss, and P. Romanelli, Phys. Lett. B 248, 146 (1990).

[119] C. Lovelace, Phys. Lett. B 135, 75 (1984).

[120] E. Fradkin and A. Tseytlin, Nucl. Phys. B 261, 1 (1985).

[121] C. Callan et al., Nucl. Phys. B 262, 593 (1985).

[122] G. Veneziano, Phys. Lett. B 265, 287 (1991).

[123] M. Gasperini and G. Veneziano, Phys.Rept. 373, 1 (2003).

[124] M. Giovannini, M. Gasperini and G. Veneziano, Phys.Lett.B 569, 113 (2003).

[125] C. P. Dettmann, N. E. Frankel, and V. Kowalenko, ıPhys. Rev. D 48, 5655 (1993).

[126] R. M. Gailis, C. P. Dettmann, N. E. Frankel, and V. Kowalenko, Phys. Rev. D 50, 3847 (1994).

[127] R. M. Gailis, N. E. Frankel, and C. P. Dettmann, Phys. Rev. D 52, 6901 (1995).

[128] R. M. Gailis and N. E. Frankel Phys. Rev. D 56, 6901 (1995).

[129] K. Holcomb and T.Tajima, Phys. Rev. D 40, 3809 (1989).

[130] T. Tajima and Taniuti, Phys. Rev. A 42, 3587 (1990).

[131] K. Subramanian and J. Barrow, Phys. Rev. D 58, 083502 (1998).

[132] K. Subramanian and J. Barrow, Phys.Rev.Lett. 81, 3575 (1998).

[133] J. Bernstein, Kinetic Theory in the Expanding Universe, (Cambridge University Press, Cambridge, England, 1988).

[134] M. Giovannini and M. Shaposhnikov, Phys. Rev. D 62, 103512 (2000). 
[135] J. Ahonen and K. Enqvist, Phys. Lett. B 382, 40 (1996).

[136] D. Boyanovsky, H. de Vega, and S. Wang Phys.Rev.D67, 065022 (2003)

[137] K. Jedamzik, V. Katalinic, and A. Olinto, Phys. Rev. D 57, 3264 (1998).

[138] D.N. Schramm and M.S. Turner, Rev. Mod. Phys. 70, 303 (1998).

[139] D. Tytler, J.M. O’Meara, N. Suzuki, and D. Lubin, Physica Scripta T85, 12 (2000).

[140] D. B. Fields and S. Sarkar, Particle Data Book Phys. Rev. D 66 , 162 (2002).

[141] H. Kurki-Suonio, e-Print Archive astro-ph/0112182.

[142] D.T. Son, Phys.Rev.D 59063008 (1999).

[143] I. Wasserman, Astrophys. J. 224, 337 (1978)

[144] P. Coles, Comments Astrophys. 16, 45 (1992).

[145] A. Brandenburg, K. Enqvist and P. Olesen Phys.Rev.D 54, 1291 (1996).

[146] A. Brandenburg, K. Enqvist and P. Olesen Phys.Lett.B 392 , 395 (1997).

[147] P. Olesen, Phys.Lett.B 398, 321 (1997).

[148] P. Olesen, NATO ASI Series B 366, 159 (1998).

[149] K. Enqvist, Int. J. Mod. Phys. D 7, 331 (1998).

[150] M. Hindmarsh and A. Everett, Phys. Rev. D, 58103505 (1998).

[151] K. Dimopoulos and A. Davis, Phys. Lett. B, 390, 87 (1996).

[152] C. Hogan, Phys. Rev. Lett., 511488 (1983).

[153] P. Saffman, Phys. Fluids 10, 1349 (1967).

[154] T. Shiromizu, Phys.Lett.B 443, 127 (1998). 
[155] A. Berera and David Hochberg, e-Print Archive cond-mat/0103447

[156] M. Christensson, M. Hindmarsh, and A. Brandenburg, Phys. Rev. E 64056405 (2001).

[157] M. Christensson, M. Hindmarsh, and A. Brandenburg, e-Print Archive astro-ph/0209119.

[158] A. Pouquet, U. Frisch and J. Léorat, J. Fluid Mech. 77, 321 (1976).

[159] J. Léorat, A. Pouquet, and U. Frisch, J. Fluid Mech. 104, 419 (1981).

[160] J. Cornwall, Phys. Rev. D56, 6146 (1997).

[161] P. Iroshnikov, Sov. Astron. 7, 566 (1964).

[162] R. Kraichnan, Phys. Fluids 8, 1385 (1965).

[163] M. Giovannini and M. Shaposhnikov, Phys. Rev. D 57, 2186 (1998).

[164] M. Giovannini and M. Shaposhnikov, Phys. Rev. Lett. 80, 22 (1998).

[165] K. Kajantie et al., Nucl. Phys. B 493, 413 (1997).

[166] V. Rubakov and A. Tavkhelidze, Phys. Lett. B 165, 109 (1985).

[167] B. Campbell, S. Davidson, J. Ellis and K. Olive, Phys. Lett. B 297, 118 (1992).

[168] L. E. Ibanez and F. Quevedo, Phys. Lett.B 283, 261 (1992).

[169] J. M. Cline, K. Kainulainen and K. A. Olive, Phys. Rev. Lett. 71, 2372 (1993).

[170] A. N. Redlich and L. C. R. Wijewardhana, Phys. Rev. Lett. 54, 970 (1984).

[171] M. Giovannini, Phys.Rev.D 61, 063004 (2000).

[172] M. Giovannini, Phys.Rev.D 61, 063502 (2000).

[173] S. Deser, R. Jackiw, and S. Templeton, Ann. Phys. 140, 372 (1982).

[174] M. Shaposhnikov, Nucl. Phys. B 287, 757 (1987). 
[175] M. Shaposhnikov, Nucl. Phys. B 288, 757 (1988).

[176] R. Jackiw and S.-Y. Pi, Phys.Rev. D 61, 105015 (2000).

[177] R. Jackiw, V.P. Nair, S-Y. Pi, Phys.Rev.D62, 085018 (2000).

[178] C. Adam, B. Muratori, and C. Nash Phys.Rev.D 61, 105018 (2000).

[179] C. Adam, B. Muratori, and C. Nash, Phys.Rev.D 62, 105027 (2000).

[180] A. Ayala, J. Besprosvany, G. Pallares, and G. Piccinelli, Phys.Rev. D 64, 123529 (2001).

[181] A. Ayala and J. Besprosvany Nucl.Phys.B 651, 211 (2003).

[182] P. Sikivie, Phys. Rev. Lett. 51, 1415 (1983).

[183] L. Maiani, R. Petronzio, and E. Zavattini, Phys. Lett. B 175, 359 (1986).

[184] M. Gasperini, Phys. Rev. Lett. 59, 396 (1987).

[185] J. Kim, Phys. Rep. 150, 1 (1987);

[186] H.-Y. Cheng, Phys. Rep. 158, 1 (1988).

[187] L. Biermann, Z. Naturf. 5A, 65 (1950).

[188] M. Langer, J.-L. Puget, and N. Aghaim, Phys. Rev. D67 043505 (2003).

[189] E. Harrison, Phys. Rev. Lett. 18, 1011 (1967).

[190] E. Harrison, Phys. Rev. 167, 1170 (1968).

[191] E. Harrison, Mon. Not. R. Astr. Soc. 147, 279 (1970).

[192] I. Mishustin and A. Ruzmaikin, Sov. Phys. JETP 34, 223 (1972).

[193] T. Vachaspati and A. Vilenkin, Phys.Rev.Lett. 67, 1057 (1991).

[194] P. Avelino and P. Shellard, Phys. Rev. D 51, 5946 (1995).

[195] K. Dimopoulos, Phys.Rev.D57, 4629 (1998).

[196] Z. Berezhiani and A. Dolgov, e-print Archive astro-ph/0305595. 
[197] A. Dolgov, e-print Archive astro-ph/0306443.

[198] C. Hogan, e-print Archive astro-ph/0005380.

[199] H. Kodama and M. Sasaki, Prog. Theor. Phys. Suppl. 78, 1 (1984).

[200] V.F. Mukhanov, H.A. Feldman, and R. H. Brandenberger, Phys. Rep. 215, 203 (1992).

[201] M. Giovannini, Class. Quant. Grav. 20, 5455 (2003).

[202] M. Gasperini, M. Giovannini, and G. Veneziano, Phys. Rev. Lett. 75, 3796 (1995).

[203] F. Finelli and A. Gruppuso, Phys. Lett. B 502, 216 (2001).

[204] L. Grishchuk, and M. Solokhin, Phys.Rev.D43, 2566 (1991).

[205] L. Grishchuk, Phys.Rev.D, 53, 6784 (1996).

[206] L. Grishchuk, and Yu. Sidorov, Phys.Rev.D 42, 3413 (1990).

[207] M. Giovannini, Phys.Rev.D 61, 087306 (2000).

[208] R. Loudon, The Quantum Theory of Light, (Oxford University Press, 1991).

[209] L. Mandel and E. Wolf, Optical Coherence and Quantum optics, (Cambridge University Press, Cambridge, England, 1995).

[210] P. Carruthers and C. C. Shih, Int. J. Mod. Phys.A 2, 1447 (1987).

[211] G. Baym, Acta Phys.Polon.B 29, 1839 (1998).

[212] R. Loudon, Rep. Prog. Phys. 43, 913 (1980).

[213] M. S. Turner and L. M. Widrow, Phys. Rev. D 37, 2734 (1988).

[214] B. Ratra, Astrophys. J. Lett. 391, L1 (1992).

[215] A. Dolgov, Phys. Rev. D 48, 2499 (1993).

[216] I. Drummond and S. Hathrell, Phys.Rev. D 22343 (1980). 
[217] S. Carroll, G. Field and R. Jackiw, Phys. Rev. D 41, 1231 (1990).

[218] W. D. Garretson, G. Field and S. Carroll, Phys. Rev. D 46, 5346 (1992).

[219] G. Field and S. Carroll Phys.Rev.D, 62, 103008 (2000).

[220] M. Giovannini, Phys. Rev. D 64, 061301 (2001).

[221] K. Bamba and J. Yokoyama, e-print Archive astro-ph/0310824.

[222] M. Gasperini, Phys. Rev. D 63, 047301 (2001)

[223] L. Okun, Sov. Phys. JETP 56, 502 (1982).

[224] O. Bertolami and D. Mota, Phys. Lett. B 455, 96 (1999).

[225] A. C. Davis and K. Dimopoulos, Phys. Rev. D 55, 7398 (1997).

[226] E. Calzetta, A. Kandus and F. Mazzitelli, Phys. Rev. D, 57, 7139 (1998).

[227] A. Kandus, E. Calzetta, F. Mazzitelli, and C. Wagner, Phys.Lett. B 472, 287 (2000).

[228] K. Dimopoulos, T. Prokopec, O. Tornkvist and A. C. Davis, Phys. Rev. $D$ 65, 063505 (2002).

[229] M. Giovannini, and M. Shaposhnikov, Proc. of CAPP2000 (July 2000, Verbier Switzerland) eprint Archive hep-ph/0011105.

[230] M. Giovannini, Phys. Rev. D 62, 123505 (2000).

[231] U. Gunther, A. Starobinsky, and A. Zhuk Phys.Rev.D69, 044003 (2004).

[232] L. H. Ford, Phys.Rev. D 31, 704 (1985).

[233] P. G. Bergmann, Int. J. Theor. Phys. 1, 25 (1968).

[234] N. D. Birrel and P. C. W. Davies, Quantum fields in curved space (Cambridge University Press, Cambridge 1982).

[235] J. Garriga and E. Verdaguer, Phys. Rev. D 39, 1072 (1991). 
[236] M. Gasperini and M. Giovannini, Phys. Lett. B282, 36 (1992); Phys.Rev.D 47, 1519 (1993).

[237] D. Lemoine and M. Lemoine, Phys.Rev. D 52, 1955 (1995).

[238] M. Giovannini, Phys.Rev. D56, 631 (1997).

[239] J. M. Cline, J. Descheneau, M. Giovannini and J. Vinet, JHEP 0306, 048 (2003)

[240] M. Giovannini, Phys.Rev.D66, 044016 (2002 ).

[241] M. Giovannini, J.-V. Le Bé and S. Riederer, Class.Quant.Grav.19, 3357 (2002).

[242] G. Baym, D. Bodeker and L. McLerran, Phys. Rev. D 53, 662 (1996).

[243] T. Kibble and A. Vilenkin, Phys. Rev. D 52, 679 (1995).

[244] P. Saffin and E. Copeland, Phys. Rev. D 56, 1215 (1997).

[245] E. Copeland, P. Saffin and O. Törnkvist, Phys. Rev. D 61, 105005 (2000).

[246] J. Ahonen and K. Enqvist, Phys. Rev. D 57, 664 (1998).

[247] O. Törnkvist, Phys. Rev. D 58, 043501 (1998).

[248] P. Kocian, e-print Archive hep-ph/0006151.

[249] V. Vlasov, V. Matveev, A. Tavkhelidze, S. Khlebnikov, M. Shaposhnikov, Sov. J. Part. Nucl. 18, 1 (1987).

[250] T. Vachaspati, Phys. Lett. B 265, 258 (1991).

[251] K. Enqvist and P. Olesen, Phys. Lett. B 329, 195 (1994).

[252] M. Joyce and M. Shaposhnikov, Phys. Rev. Lett. 79, 1193 (1997).

[253] K. Kajantie, M. Laine, K. Rummukainen, and M. E. Shaposhnikov, Phys. Rev. Lett. 77, 2887 (1996).

[254] K. Kajantie, M. Laine, K. Rummukainen, and M. E. Shaposhnikov, Nucl. Phys. B 458, 90 (1996). 
[255] J. Quashnock, A. Loeb and D. Spergel, Astrophys. J. Lett. 344, L49 (1989).

[256] B. Cheng and A. Olinto, Phys. Rev. D 50, 2421 (1994).

[257] G. Sigl, A. Olinto, and K. Jedamzik, Phys. Rev. D 55, 4852 (1997).

[258] D. Boyanovsky, M. Simionato, and H. de Vega, Phys.Rev.D 67, 023502 (2003).

[259] D. Boyanovsky, H. de Vega, and M. Simionato, Phys.Rev.D 67, 123505 (2003).

[260] D. Boyanovsky, H. de Vega, and M. Simionato, e-Print Archive astro-ph/0305131.

[261] P. Elmfors, K. Enqvist, K. Kainulainen, Phys.Lett.B 440, 269 (1998).

[262] K. Kajantie, M. Laine, J. Peisa, K. Rummukainen, and M. E. Shaposhnikov, Nucl.Phys.B544, 357 (1999).

[263] G. Volovik, Phys.Rept. 351, 195 (2001)

[264] G. Greenstein, Nature 223, 938 (1969).

[265] J. J. Matese and R. F. O' Connel, Astrophys. J. 160, 451 (1970).

[266] B. Cheng, D. N. Schramm and J. Truran, Phys. Rev. D 45, 5006 (1994).

[267] B. Cheng, A. Olinto, D. N. Schramm and J. Truran, Phys. Rev. D 54, 4174 (1996).

[268] P. Kernan, G. Starkman and T. Vachaspati, Phys. Rev. D 54, 7207 (1996).

[269] P. Kernan, G. Starkman and T. Vachaspati, Phys. Rev. D 56, 3766 (1996).

[270] D. Grasso and H. Rubinstein, Astropart. Phys. 3, 95 (1995).

[271] D. Grasso and H. Rubinstein, Phys. Lett. B 379, 73 (1996).

[272] K. Thorne, Astrophys. J. 148, 51 (1967). 
[273] S. Hawking and R. J. Tayler, Nature309, 1278 (1966).

[274] J. Barrow, Mon. Not. R. Astron. Soc.175, 359 (1976).

[275] J. Barrow, Phys.Rev.D 55, 7451 (1997)

[276] G. Steigman, Ann. Rev. Astron. Astrophys. 14, 339 (1976).

[277] J. Rehm and K. Jedamzik, Phys. Rev. Lett. 81, 3307 (1998).

[278] H. Kurki-Suonio and E. Sihvola, Phys. Rev. Lett. 84, 3756 (2000).

[279] H. Kurki-Suonio and E. Sihvola, Phys. Rev. D62, 103508 (2000).

[280] E. Sihvola, Phys. Rev. D 63, 103001 (2001).

[281] H. Kurki-Suonio, BBN calculations, astro-ph/0112182.

[282] J. Rehm and K. Jedamzik, Phys. Rev. D63, 043509 (2001).

[283] M. Giovannini, H. Kurki-Suonio and E. Shivola, Phys. Rev. D 66, 043504 (2002).

[284] Ya. Zeldovich and I. Novikov, The Structure and Evolution of the Universe, (Chicago University Press, Chicaggo, 1971), Vol.2.

[285] Ya. Zeldovich, Sov. Phys. JETP21, 656 (1965).

[286] L. Grishchuk, A. Doroshkevich, and I. Novikov Sov. Phys. JETP28, 1210 (1969).

[287] K. Jedamzik, V. Katalinic, and A. V. Olinto, Phys. Rev. Lett. 85 700, (2000).

[288] J.Adams, U.Danielsson, D.Grasso, and H. Rubinstein, Phys. Lett. B 388, 253 (1996).

[289] M. Gasperini, M. Giovannini, and G. Veneziano, Phys.Rev.D, 52, 6651 (1995).

[290] R. Durrer, M. Gasperini, M. Sakellariadou and G. Veneziano, Phys. Rev. D59, 43511 (1999).

[291] V. Bozza, et al. Phys. Rev. D 67, 063514 (2003). 
[292] R. K. Sachs and A. M. Wolfe, Astrophys. J. 147, 73 (1967).

[293] C. L. Bennet et al., Astrophys. J. Lett. 464, L1 (1996).

[294] M. Tegmark, Astrophys. J. Lett. 464, L35 (1996).

[295] C. B. Netterfield et al., Astrophys. J. 571, 604 (2002).

[296] N. W. Halverson et al., Astrophys. J. 568, 38 (2002).

[297] A. T. Lee et al., Astrophys. J. 561, L1 (2001).

[298] A. Benoit et al., astro-ph/0210305.

[299] C. Bennett et al. Astrophys.J.Suppl.148 1 (2003).

[300] D. Spergel et al. Astrophys.J.Suppl. 148, 175 (2003).

[301] G. Hinshaw et al., Astrophys.J.Suppl. 148, 135 (2003).

[302] S. Koh, C. Lee, Phys.Rev.D 62, 083509 (2000).

[303] J. Barrow, P. Ferreira, and J. Silk, Phys. Rev. Lett. 78, 3610 (1997).

[304] A. Mack, T. Kahniashvili, and A. Kosowsky, Phys. Rev. D 65, 123004 (2002).

[305] R. Durrer, T. Kahniashvili, and A. Yates, Phys.Rev.D 58 123004, (1998).

[306] R. Durrer, T. Kahniashvili, Helv.Phys.Acta 71, 445 (1998).

[307] R. Durrer, P. Ferreira, and T. Kahniashvili, Phys.Rev.D 61, 043001 (2000).

[308] C. Caprini and R. Durrer, Phys.Rev.D 65, 023517 (2002).

[309] T. Seshadri and K. Subramanian, Phys.Rev.Lett. 87, 101301 (2001).

[310] K. Subramanian, J. Barrow, Mon. Not. Roy. Astron. Soc. 335, L57 (2002).

[311] K. Subramanian, T. Seshadri, and J. Barrow, Mon. Not. Roy. Astron. Soc. 344, L31 (2003). 
[312] L. Pogosian, T. Vachaspati, S. Winitzki Phys.Rev.D 65, 083502 (2002).

[313] M. Giovannini, Phys. Rev. D56, 3198 (1997).

[314] M. Rees, Astrophys. J. 153, L1 (1968).

[315] P. M. Lubin and G. F. Smoot, Astrophys. J. 245, 1 (1981).

[316] P. M. Lubin, P. Melese and G. F. Smoot, Astrophys. J. Lett. 273, L51 (1983).

[317] M. Bersanelli et. al. http://www.mrao.cam.ac.uk/projects/cpac/.

[318] E. Milaneschi and R. Fabbri, Astron. Astrophys. 151, 7 (1985).

[319] D. Coulson, R. Crittenden and N. G. Turok, Phys. Rev. D 52, 5402 (1995).

[320] D. Coulson, R. Crittenden and N. G. Turok, Phys. Rev. Lett. 73, 2390 (1994).

[321] A. Kosowsky and A. Loeb, Astrophys. J461, 1 (1996).

[322] D. Harari, J. Hayward and M. Zaldarriaga, Phys. Rev. D 55, 1841 (1997).

[323] D. Deryagin, D. Grigoriev, V. Rubakov and M. Sazhin, Mod. Phys. Lett. A 11, 593 (1986).

[324] M. Giovannini, Phys. Rev. D 58, 083504 (1998).

[325] M. Giovannini, Phys. Rev. D 60, 123511 (1999).

[326] M. Giovannini, Class.Quant.Grav. 16, 2905 (1999).

[327] D. Babusci and M. Giovannini, Int. J. Mod. Phys. D 10, 477 (2001).

[328] D. Babusci and M. Giovannini, Class. Quant. Grav. 17, 2621 (2000).

[329] C. Tsagas and J. Barrow, Class. Quantum Grav. 14, 2539 (1997).

[330] C. Tsagas and J. Barrow, Class. Quantum Grav. 15, 3523 (1998).

[331] D. Papadopoulos Class.Quant.Grav. 19, 1 (2002). 
[332] M. Giovannini, Phys. Rev. D 59, 123518 (1999).

[333] M. Ryan and L. Shepley, Homogeneous Relativistic Cosmologies, (Princeton University Press, Princeton 1978).

[334] R. Kantowski and P. K. Sachs. J. Math. Phys. 7, 443 (1966).

[335] J. Barrow and M. Dabrowski, Phys. Rev. D55, 630 (1997).

[336] J. Barrow and K. Kunze, Phys. Rev. D55, 623 (1997).

[337] M. Giovannini, Phys. Rev. D 62, 067301 (2000).

[338] L. Ford, Phys. Rev. D40, 967 (1989).

[339] A. Burd and J. Lidsey, Nucl. Phys. B351, 679 (1991).

[340] J. Lidsey, Nucl. Phys. B351, 695 (1991). 